\title{
The Free-Fermion Eight-Vertex Model: Couplings, Bipartite Dimers and Z-Invariance
}

\author{
Paul Melotti iD \\ Université de Fribourg, Fribourg, Switzerland. E-mail: paul.melotti@unifr.ch
}

Received: 6 November 2018 / Accepted: 18 September 2020

Published online: 17 November 2020 - (C) The Author(s) 2020

\begin{abstract}
We study the eight-vertex model at its free-fermion point. We express a new "switching" symmetry of the model in several forms: partition functions, order-disorder variables, couplings, Kasteleyn matrices. This symmetry can be used to relate freefermion $8 \mathrm{~V}$-models to free-fermion $6 \mathrm{~V}$-models, or bipartite dimers. We also define new solution of the Yang-Baxter equations in a "checkerboard" setting, and a corresponding $Z$-invariant model. Using the bipartite dimers of Boutillier et al. (Probab Theory Relat Fields 174:235-305, 2019), we give exact local formulas for edge correlations in the $\mathrm{Z}$-invariant free-fermion $8 \mathrm{~V}$-model on lozenge graphs, and we deduce the construction of an ergodic Gibbs measure.
\end{abstract}

\section{Contents}

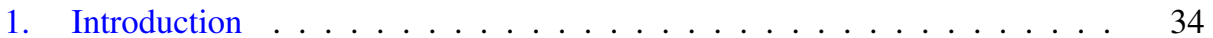

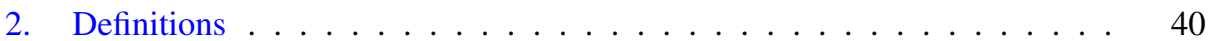

2.1 Eight-vertex-model . . . . . . . . . . . . . . . . . . . . 40

2.2 Ising model . . . . . . . . . . . . . . . . . . . . . . 43

2.3 Dimer model . . . . . . . . . . . . . . . . . . . . . . 43

2.4 Order and disorder variables . . . . . . . . . . . . . . 43

2.4.1 Ising correlators . . . . . . . . . . . . . . . . . . . . . . . 44

$2.4 .28 \mathrm{~V}$ correlators . . . . . . . . . . . . . . . . . . . . 44

3. Couplings of 8V-Models . . . . . . . . . . . . . . . . . 45

3.1 Spin-vertex correspondence . . . . . . . . . . . . . . . . 45

3.2 Modifications of weights . . . . . . . . . . . . . . . . . 46

3.3 Free-fermion $8 \mathrm{~V}$ correlators . . . . . . . . . . . . . . 47

3.4 Coupling of free-fermion $8 \mathrm{~V}$-models . . . . . . . . . . . . . . . . 48

4. Kasteleyn Matrices . . . . . . . . . . . . . . . . . . . . . . . . . . . . 49

4.1 Free-fermion $8 \mathrm{~V}$ to dimers . . . . . . . . . . . . . . . 50

4.2 Skew-symmetric real matrix ............... 51 


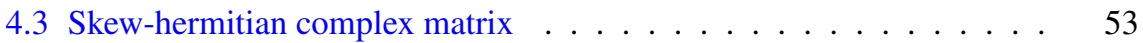

4.4 Eight-vertex partition function and correlations $\ldots \ldots \ldots \ldots . . \ldots 56$

4.5 Relations between matrices $K_{\alpha, \beta}^{-1} \ldots \ldots \ldots \ldots \ldots \ldots$

4.5.1 Spherical and planar cases . . . . . . . . . . . . 57

4.5 .2 Toric case . . . . . . . . . . . . . . . . . . . . . 59

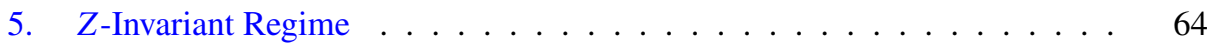

5.1 Checkerboard Yang-Baxter equations . . . . . . . . . . . 64

5.2 Lozenge graphs . . . . . . . . . . . . . . . . . 65

5.3 Local expression for $K_{k, l}^{-1} \ldots \ldots \ldots \ldots \ldots \ldots \ldots$

5.3 .1 Inverse of $\mathcal{K}_{k} \ldots \ldots \ldots \ldots \ldots \ldots \ldots \ldots$

5.3 .2 Inverse of $K_{k, l} \quad \ldots \ldots \ldots \ldots \ldots \ldots$

5.3.3 Asymptotics of coefficients . . . . . . . . . . . . . . . . . 69

5.4 Free energy and Gibbs measure . . . . . . . . . . . . . 70

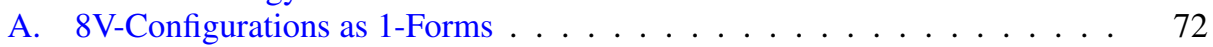

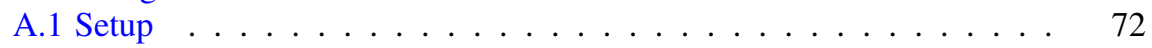

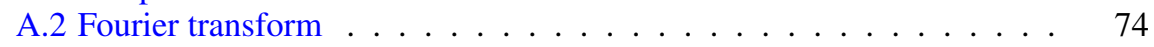

A.3 Correlators . . . . . . . . . . . . . . . . . . . . . 74

B. Proof of Lemma $35 \ldots \ldots \ldots \ldots \ldots \ldots$

\section{Introduction}

The eight-vertex model, or 8V-model for short, was introduced by Sutherland [63] and Fan and $\mathrm{Wu}[30]$ as a generalization of the 6V-model, which itself finds its origins in the study of square ice $[53,61]$. The configurations of the $8 \mathrm{~V}$-model are orientations of the edges of $\mathbb{Z}^{2}$ such that every vertex has an even number of incoming edges, like in Fig. 1. There are eight possible local configurations, hence the name. In the most classical setting, the model is characterized by four local weights $a, b, c, d$ such that opposite local configurations are given the same weight, see [6].

This model attracted attention during the 70s and 80s, and was famously solved on the square lattice and a few other regular lattices using transfer matrices methods, see again [6] and references therein. It exhibits phenomena that were surprising at the time; for instance, it has been predicted that some critical exponents depend continuously on $a, b, c, d$. This is the case of the exponent $v$ for the correlation length: in a generic disordered phase, with inverse temperature $\beta$, the edge-edge correlations between faraway edges $e, e^{\prime}$ should decay as $\exp \left(-\frac{\left|e-e^{\prime}\right|}{\xi(\beta)}\right)$. As the system becomes critical one should have $\xi(\beta) \sim\left(\beta-\beta_{c}\right)^{-\nu}$. It seems that these predictions and the computation of $v$ still require mathematical investigation. In what follows, we focus on the special free-fermion case $a^{2}+b^{2}=c^{2}+d^{2}$ on more generic lattices. A consequence of the techniques developed here is a construction of a Gibbs measure on these graphs and a proof that $v=1$ for free-fermion models, in accordance with Baxter's computation.

Let us now describe our setting. A configuration can be represented equivalently as a polygon, by choosing a checkerboard coloring of the faces of $\mathbb{Z}^{2}$ and drawing in bold the edges oriented with, say, a white face on their left. In this paper we extend definitions to graphs that are dual of a planar quadrangulation $\mathcal{Q}$, whose set of vertices (resp. edges, faces) we denote by $\mathcal{V}$ (resp. $\mathcal{E}, \mathcal{F}$ ); an example is displayed in Fig. 5. More precisely, the Boltzmann weight of a configuration is the product of local weights associated to local configurations at a face $f$ of the quadrangulation $\mathcal{Q}$, as in Fig. 4, that are denoted $A(f), B(f), C(f), D(f)$ (we use uppercase notations to emphasize 

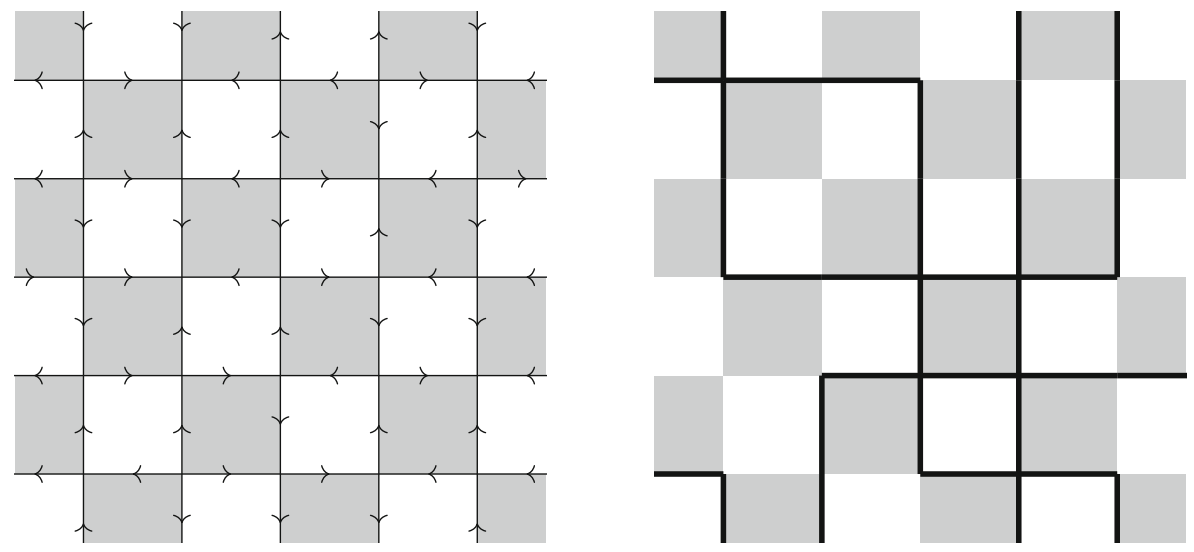

Fig. 1. Two equivalent representations of an eight-vertex configuration on $\mathbb{Z}^{2}$

that these are functions of the faces). The case of a $6 \mathrm{~V}$-model corresponds to $D(f)=0$ at every face. Notice that complementary configurations have the same weight, which means that we are in a "zero field" case. To make these weights well-defined, notice also that we fixed a bipartite coloring of $\mathcal{Q}$. This is sometimes referred to as a checkerboard model [3,7], or a staggered model in the case of the square lattice [37]. Checkerboard (or alternating, or staggered) $8 \mathrm{~V}$ models have attracted some attention, in particular for their relation with the Ashkin-Teller model [6,67], but little is known about them in general. In this paper we investigate checkerboard $8 \mathrm{~V}$-models that satisfy the freefermion condition:

$$
A^{2}+B^{2}=C^{2}+D^{2}
$$

Under this condition, the theory of transfer matrices can be adapted, making computation easier than for the complete 8V-model [8-10,31-33]. There exists a different method using a correspondence with dimers on a non-bipartite decorated graph, leading to the computation of Pfaffians [30,37,54-56]. The dimer model is an object of interest of its own in the mathematical community, an important example being the celebrated description of Gibbs measures and phase diagram of bipartite dimers on periodic graphs $[21,48]$. Adapting these results to non-bipartite settings is an important open problem in the community, making the previous correspondence of $8 \mathrm{~V}$ configurations to nonbipartite dimers of little help for the description of, say, Gibbs measures and correlations. In what follows we provide a way to transform these non-bipartite dimers into bipartite ones. From the point of view of the dimer model, unexpectedly, this provides a family of examples of non-bipartite dimers whose statistics can be related to bipartite ones, and whose spectral curve is reducible, as shown in formula (2).

Our method is based on a "switching" result, that we introduce now. If we perform a gauge transformation by multiplying all weights $A(f), B(f), C(f), D(f)$ at a face $f$ by the same constant, the relative weight of different $8 \mathrm{~V}$-configurations are unchanged. Thus an $8 \mathrm{~V}$ model with weights satisfying the free-fermion condition (1) can be effectively represented by two free parameters per face, say $\alpha(f), \beta(f) \in \mathbb{R} / 2 \pi \mathbb{Z}$; see (10) for the exact parametrization. Our parametrization is such that when $\alpha=\beta$, the model becomes a $6 \mathrm{~V}$ one. We denote by $X_{\alpha, \beta}$ the whole set of weights corresponding to $\alpha, \beta$, and by $\mathcal{Z}_{8 V}\left(\mathcal{Q}, X_{\alpha, \beta}\right)$ the partition function; when $\alpha=\beta$, we denote it by $\mathcal{Z}_{6 V}\left(\mathcal{Q}, X_{\alpha, \alpha}\right)$ to 
emphasize that it becomes a $6 \mathrm{~V}$-model. The choice of parameters $\alpha, \beta$ is such that we have the following "switching" relation, see Theorem 15 for a generalized statement and (20) for the value of the constant $c_{\alpha, \beta}$ :

Theorem 1. Let $\mathcal{Q}$ be a quadrangulation of the sphere. For any $\alpha, \beta, \alpha^{\prime}, \beta^{\prime}: \mathcal{F} \rightarrow$ $\left(0, \frac{\pi}{2}\right)$,

$$
\frac{\mathcal{Z}_{8 V}\left(\mathcal{Q}, X_{\alpha, \beta}\right)}{\sqrt{c_{\alpha, \beta}}} \frac{\mathcal{Z}_{8 V}\left(\mathcal{Q}, X_{\alpha^{\prime}, \beta^{\prime}}\right)}{\sqrt{c_{\alpha^{\prime}, \beta^{\prime}}}}=\frac{\mathcal{Z}_{8 V}\left(\mathcal{Q}, X_{\alpha, \beta^{\prime}}\right)}{\sqrt{c_{\alpha, \beta^{\prime}}}} \frac{\mathcal{Z}_{8 V}\left(\mathcal{Q}, X_{\alpha^{\prime}, \beta}\right)}{\sqrt{c_{\alpha^{\prime}, \beta}}} .
$$

In particular, for $\left(\alpha^{\prime}, \beta^{\prime}\right)=(\beta, \alpha)$, we recover $6 \mathrm{~V}$-models on the right-hand side. This gives a new relation between free-fermion $8 \mathrm{~V}$-models and $6 \mathrm{~V}$ ones:

Corollary 2. Let $\mathcal{Q}$ be a quadrangulation of the sphere. For any $\alpha, \beta: \mathcal{F} \rightarrow\left(0, \frac{\pi}{2}\right)$,

$$
\left(\mathcal{Z}_{8 V}\left(\mathcal{Q}, X_{\alpha, \beta}\right)\right)^{2}=\frac{c_{\alpha, \beta}}{\sqrt{c_{\alpha, \alpha} c_{\beta, \beta}}} \mathcal{Z}_{6 V}\left(\mathcal{Q}, X_{\alpha, \alpha}\right) \mathcal{Z}_{6 V}\left(\mathcal{Q}, X_{\beta, \beta}\right) .
$$

This illustrates how the switching identity of Theorem 1 can turn useful, as freefermion $6 \mathrm{~V}$ models are related to bipartite dimers. Theorem 1 also suggests that other hidden features of free-fermion $8 \mathrm{~V}$-models might exist. We identify several of them.

First, it hints at a possible coupling of pairs of $8 \mathrm{~V}$-configurations. If $\tau, \tau^{\prime}$ are two $8 \mathrm{~V}$-configurations, seen as subsets of the dual edges of $\mathcal{Q}$, their XOR is still an $8 \mathrm{~V}$ configuration; we denote it by $\tau \oplus \tau^{\prime}$. To ensure that the $8 \mathrm{~V}$-weight actually define a probability measure, we impose a sufficient condition (13) on the parametrization. We prove the following:

Theorem 3. Let $\mathcal{Q}$ be a quadrangulation of the sphere, and let $\alpha, \beta, \alpha^{\prime}, \beta^{\prime}: \mathcal{F} \rightarrow\left(0, \frac{\pi}{2}\right)$ be such that $(\alpha, \beta),\left(\alpha^{\prime}, \beta^{\prime}\right),\left(\alpha, \beta^{\prime}\right),\left(\alpha^{\prime}, \beta\right)$ all satisfy (13). Let $\tau_{\alpha, \beta}, \tau_{\alpha^{\prime}, \beta^{\prime}}, \tau_{\alpha, \beta^{\prime}}, \tau_{\alpha^{\prime}, \beta}$ be independent $8 V$-configurations with the corresponding Boltzmann distributions. Then $\tau_{\alpha, \beta} \oplus \tau_{\alpha^{\prime}, \beta^{\prime}}$ and $\tau_{\alpha, \beta^{\prime}} \oplus \tau_{\alpha^{\prime}, \beta}$ are equal in distribution.

Theorem 3 is proved via the formalism of order-disorder variables [26,40], see Theorem 15 for a generalized statement. By specifying again to $\left(\alpha^{\prime}, \beta^{\prime}\right)=(\beta, \alpha)$ it implies that the XOR of two independent $8 \mathrm{~V}$-configurations (with the same distribution) is distributed as the XOR of two independent $6 \mathrm{~V}$-configurations (with different distributions). This may be worth investigating from the point of view of conformal objects in the limit: the XOR of free-fermion 6V-models are effectively double-dimer loops for two independent, differently distributed (bipartite) dimers configurations. In the identically distributed case, these are conjectured to converge to CLE(4) $[2,25,47]$, but the differently-distributed case seems more mysterious at the moment. In any case, the potential occurrence of such conformal ensembles in the context of $8 \mathrm{~V}$-models is surprising.

Although being unexpected, this XOR property is reminiscent of the coupling identities of $[15,26]$. However these previous identities involved two independent Ising models, while our results are naturally associated with four Ising models (see Corollary 14) and cannot be deduced immediately from their work.

Second, it is natural to wonder what happens if $\mathcal{Q}$ is a quadrangulation of the torus. This is useful in particular to understand periodic boundary conditions and construct infinite measures on the full plane, as we explain later. In the toric case, the $8 \mathrm{~V}$-weights $X_{\alpha, \beta}$ are naturally associated with a characteristic (Laurent) polynomial of two complex variables, denoted $P_{\alpha, \beta}^{8 V}(z, w)$, and defined in (23) just like in the case of the dimer model [48]. The analogous statement of Theorem 1 is the following, which works under milder hypothesis (12) (see Theorem 27 for a complete statement): 

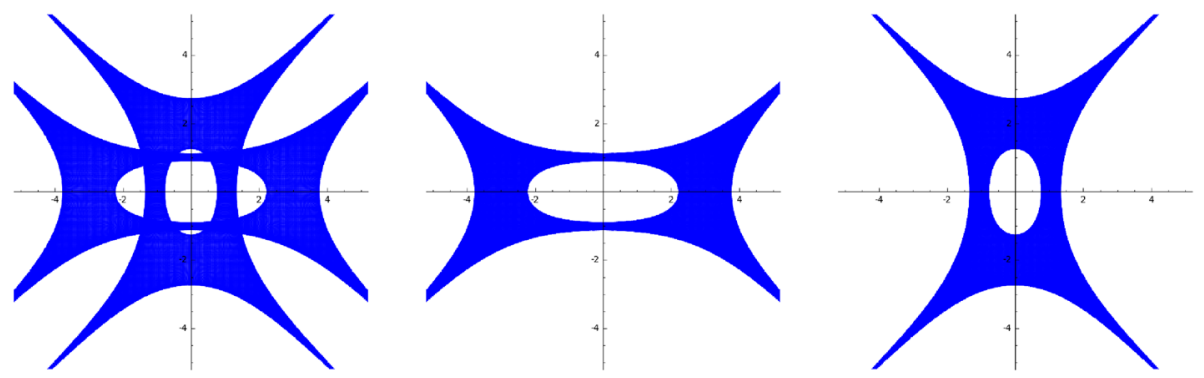

Fig. 2. Amoebas of the curves defined by $P_{\alpha, \beta}^{8 V}, P_{\alpha}^{6 V}$ and $P_{\beta}^{6 V}$ for the square lattice

Theorem 4. Let $\mathcal{Q}$ be a quadrangulation of the torus. Let $\alpha, \beta, \alpha^{\prime}, \beta^{\prime}: \mathcal{F} \rightarrow[0,2 \pi)$ be such that $(\alpha, \beta)$ and $\left(\alpha^{\prime}, \beta^{\prime}\right)$ satisfy (12). Then

$$
c_{\alpha, \beta} c_{\alpha^{\prime}, \beta^{\prime}} P_{\alpha, \beta}^{8 V}(z, w) P_{\alpha^{\prime}, \beta^{\prime}}^{8 V}(z, w)=c_{\alpha, \beta^{\prime}} c_{\alpha^{\prime}, \beta} P_{\alpha, \beta^{\prime}}^{8 V}(z, w) P_{\alpha^{\prime}, \beta}^{8 V}(z, w) .
$$

In particular (see Corollary 29),

$$
P_{\alpha, \beta}^{8 V}(z, w)=\tilde{c} P_{\alpha}^{6 V}(z, w) P_{\beta}^{6 V}(z, w) .
$$

The polynomials $P_{\alpha}^{6 V}$ and $P_{\beta}^{6 V}$ correspond to bipartite dimers [15,26,58,69]. The curves defined by their zero locus in $\mathbb{C}^{2}$ are Harnack curves [48]. Thus the zero locus of $P_{\alpha, \beta}^{8 V}$ is the union of two Harnack curves. This can be observed in the amoebae of Fig. 2, (the amoeba is the image of the zero locus under the map $(z, w) \mapsto(\log |z|, \log |w|))$.

Third, the $8 \mathrm{~V}$-models at the free-fermion point corresponds to non-bipartite dimers, for which we can define a version of a Kasteleyn matrix $K_{\alpha, \beta}$, see Sect. 4.3. The elements of the inverse of $K_{\alpha, \beta}$ are related to the correlations of the $8 \mathrm{~V}$-model (see Proposition 22), and thus encode the physical behaviour of the model. It is possible to get a relation between those inverse matrices; precise statements are given in Theorem 23 on the sphere and in Theorem 26 on the torus, and the matrix $T$ is defined by (30).

\section{Theorem 5.}

$$
K_{\alpha, \beta}^{-1}=\frac{1}{2}\left((I+T) K_{\alpha, \beta^{\prime}}^{-1}+(I-T) K_{\alpha^{\prime}, \beta}^{-1}\right) .
$$

This has the remarkable property of holding for all $\alpha^{\prime}, \beta^{\prime}$, even though this is not apparent in the right-hand-side. Again we can set $\left(\alpha^{\prime}, \beta^{\prime}\right)=(\beta, \alpha)$, so that this formula relates $8 \mathrm{~V}$-correlations to $6 \mathrm{~V}$ ones, i.e. to bipartite dimers $[15,26,58,69]$. We give several consequences of this identity with the solution of the $8 \mathrm{~V}$-model in the $Z$-invariant regime on lozenge graphs.

A model is said to be Z-invariant when it satisfies a form of the Yang-Baxter equations, or a star-triangle transformation (see Fig. 15). In the approach via transfer matrices, this property is often seen as a sufficient condition for the commutativity of transfer matrices [6], see also [11,29]. However it has also been shown by Baxter that the star-triangle move is enough information to guess the behavior of the model on very generic lattices [5], without relying on transfer matrices methods. In particular, it should imply a form of locality for the model, which means that the two-point correlations depend only on a path (any path) between the two points. This property is surprising, since in general 

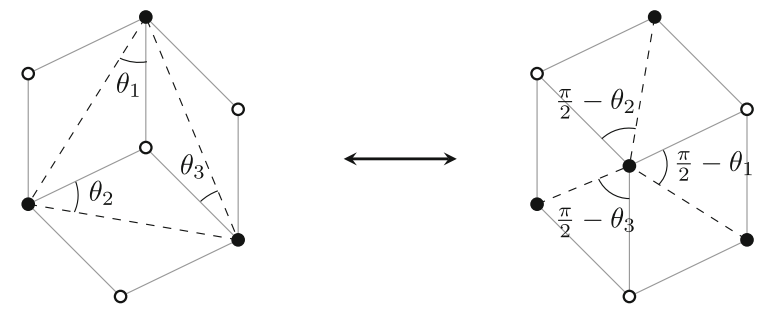

Fig. 3. Star-triangle move on a lozenge graph. The angles satisfy $\theta_{1}+\theta_{2}+\theta_{3}=\frac{\pi}{2}$

correlations are expected to depend on the geometry of the whole graph. We prove that it holds for $Z$-invariant free-fermion models on lozenge graphs, which we introduce now.

One way to interpret $Z$-invariance geometrically is to use isoradial graphs. For these graphs, the faces of the quadrangulation $\mathcal{Q}$ are supposed to be rhombi with the same edge-length (we call $\mathcal{Q}$ a lozenge graph), and the weights $A(f), B(f), C(f), D(f)$ at a face $f$ are supposed to depend on the half-angle $\theta$ of the rhombus $f$ at the black vertices. The angles $\theta$ satisfy some relations under star-triangle transformation (see Fig. 3), and the goal is to define Boltzmann weights in terms of $\theta$ so as to transform these relations into the Yang-Baxter equations. Several $Z$-invariant models have been studied on lozenge graphs, including the bipartite dimer model [45], Ising model [13,16], Laplacian (or spanning forest model) [14,45], random cluster model [28]. The results of [49] also imply that we do not lose anything by considering the Yang-Baxter equations on a lozenge graph rather than on a pseudoline arrangement, like that of [5].

The $Z$-invariant weights of the $8 \mathrm{~V}$ model in the non-checkerboard case have been parameterized by Baxter [4-6] and Zamolodchikov [70]. Other techniques have appeared since to classify these solutions $[35,50,62,66]$. By considering checkerboard YangBaxter equations, more solutions can appear, as noted for instance in [59], although no complete parametrization is known. Here we introduce what seems to be the first set of checkerboard $Z$-invariant weights for the $8 \mathrm{~V}$ model, all included in the free-fermion case.

Let $k, l$ be complex numbers such that $k^{2}, l^{2} \in(-\infty, 1)$. For any real number $x$ let $x_{k}=\frac{2 \mathrm{~K}(k)}{\pi} x$, where $\mathrm{K}(k)$ is the complete elliptic integral of the first kind, and similarly for $x_{l}$. Then the following $8 \mathrm{~V}$-weights of lozenge graphs, expressed in terms of Jacobi's elliptic functions (see [1,52]) at a face $f$ with half-angle $\theta$, satisfy the Yang-Baxter equations:

$$
\begin{aligned}
& A(f)=\operatorname{sn}\left(\theta_{k} \mid k\right)+\operatorname{sn}\left(\theta_{l} \mid l\right) \\
& B(f)=\operatorname{cn}\left(\theta_{k} \mid k\right)+\operatorname{cn}\left(\theta_{l} \mid l\right) \\
& C(f)=1+\operatorname{sn}\left(\theta_{k} \mid k\right) \operatorname{sn}\left(\theta_{l} \mid l\right)+\operatorname{cn}\left(\theta_{k} \mid k\right) \mathrm{cn}\left(\theta_{l} \mid l\right) \\
& D(f)=\operatorname{cn}\left(\theta_{k} \mid k\right) \operatorname{sn}\left(\theta_{l} \mid l\right)-\operatorname{sn}\left(\theta_{k} \mid k\right) \operatorname{cn}\left(\theta_{l} \mid l\right)
\end{aligned}
$$

We prove this in Proposition 31. When $\left(1-k^{2}\right)\left(1-l^{2}\right)=1$ (or $k^{*}=l$ in the notations of [16]) the weights no longer depend on the bipartite coloring of $\mathcal{Q}$ (i.e if a face $f$ has a half-angle $\theta$ and $g$ has a half-angle $\frac{\pi}{2}-\theta$, then $A(f)=B(g)$, etc.), and we recover Baxter's solution in the free-fermion case. When $k=l$ we get a $Z$-invariant $6 \mathrm{~V}$ model whose corresponding dimer model can be found in [16]. At this point it is unknown if this new parametrization of checkerboard Yang-Baxter equations could be extended outside of the free-fermion manifold. 
We prove that this choice of local weights indeed provides a Gibbs measure with the locality property, confirming in that case the prediction of Baxter. To be more precise, every edge of the lozenge graph $e \in \mathcal{E}$ is associated with two vertices $\mathrm{b}_{e}, \mathrm{w}_{e}$ that correspond to the "leg" of a decorated graph $G^{T}$, see Fig. 8. In Sect. 5 we introduce a local operator $K_{k, l}^{-1}$ on the vertices of $G^{T}$. It satisfies the following:

Theorem 6. Let $\mathcal{Q}$ be a planar lozenge graph. For any $0 \leq k<l<1$, there exists a unique probability measure $\mathcal{P}_{8 \mathrm{~V}}$ on the space of $8 \mathrm{~V}$-configurations equipped with the $\sigma$-field generated by cylinders. It is such that for any $e_{1}, \ldots, e_{p} \in \mathcal{E}$, let $\mathrm{V}$ be the associated vertices of $G^{T}: \mathrm{V}=\left\{\mathrm{b}_{1}, \mathrm{w}_{1}, \ldots, \mathrm{b}_{p}, \mathrm{w}_{p}\right\}$, then

$$
\mathcal{P}_{8 V}\left(e_{1}, \ldots, e_{m} \in \tau\right)=\sqrt{\operatorname{det}\left[\left(K_{k, l}^{-1}\right)_{V}\right]} .
$$

\section{Moreover, $\mathcal{P}$ is a translation invariant ergodic Gibbs measure.}

The construction is based on the switching property in operator form from Theorem 5 and on the work on bipartite dimers of [16]. When $\mathcal{Q}$ is $\mathbb{Z}^{2}$-periodic, it yields the free energy (51). It also allows for the computation of asymptotics of coefficients, using results from [16]: under some technical hypothesis, we show that when $0<k<l<1$ the coefficients of the inverse Kasteleyn matrix between points at distance $r$ decays as $r^{-\frac{1}{2}} e^{-r / \zeta_{k}}$ (see Corollary 37). Notice that the effect of $l$ vanishes in the asymptotics. When $k=0$ the decay is polynomial, corresponding to a critical model. When $k \rightarrow 0$, we prove that the quantity $\zeta_{k}$ is a $\Theta\left(k^{-2}\right)$ in Proposition 38. As $k^{2}$ plays the role of $\left(\beta-\beta_{c}\right)$ in usual statistical mechanics terms, this critical exponent is compatible with that of the correlation length, $v=1$, in the universality class of the Ising model, and with previous predictions [6].

Outline of the paper. In Sect. 2 we properly define the 8V, Ising and dimer models in spherical, toric and planar settings. We also introduce the formalism of order-disorder correlators.

In Sect. 3 we restrict ourselves to the spherical case. We compute the correlators of free-fermion 8V models and relate them to Ising ones, generalizing results of [26], see Corollary 14. We prove the coupling result of Theorem 3, first in correlators terms (Theorem 15), then in probabilistic terms; the latter is deduced from the former by using a discrete Fourier transformation described in Appendix A. The results of Sect. 3 imply Theorem 1. Sects. 4 and 5 are independent of Sect. 3.

In Sect. 4 we define the dimer model associated to the $8 \mathrm{~V}$-model, and appropriate versions of Kasteleyn matrices, one being skew-symmetric and the other being skewhermitian; they are related by a diagonal conjugation, see Lemma 20. We show that the edge correlations can be expressed as minors of the inverse Kasteleyn matrices, see Proposition 22. We prove the relation of inverses of Theorem 5. This gives an alternative proof of Theorem 1, as well as its toric counterpart, Theorem 4.

In Sect. 5 we prove that the weights (3) satisfy the Yang-Baxter equations. Using the relation to $6 \mathrm{~V}$ models coming from Sect. 4 , we give a local formula for the inverse Kasteleyn matrix $K_{k, l}^{-1}$ in the full plane in Sect. 5.3. We prove the mentioned asymptotics and critical exponent in Corollary 37 and Proposition 38. Finally we construct an ergodic Gibbs measure in the $Z$-invariant case in Sect. 5.4 and prove Theorem 6. 

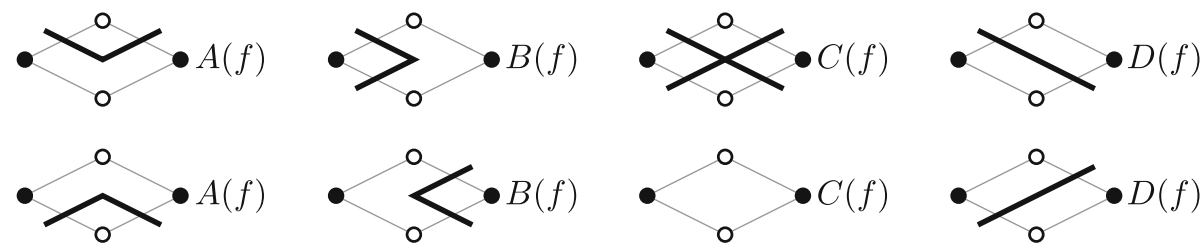

Fig. 4. The eight possible configurations for $\tau$ at a face $f \in \mathcal{F}$ and their local weight $w_{f}(\tau)$

\section{Definitions}

Let $\mathcal{Q}$ be a quadrangulation of a surface $\mathcal{S}$, that is a finite connected graph $\mathcal{Q}=(\mathcal{V}, \mathcal{E})$, without multi-edges and self-loops, embedded on $\mathcal{S}$ so that edges do not intersect, and so that the faces of $\mathcal{Q}$ are homeomorphic to disks and have degree 4 . We denote by $\mathcal{F}$ its set of faces. We will focus on three cases:

- the spherical case where $\mathcal{S}$ is the two-dimensional sphere and $\mathcal{Q}$ is finite;

- the planar case where $\mathcal{S}$ is $\mathbb{R}^{2}$ and $\mathcal{Q}$ is infinite and covers the whole plane;

- the toric case where $\mathcal{S}$ is the two-dimensional torus and $\mathcal{Q}$ is finite and bipartite.

Since $\mathcal{Q}$ is bipartite in all these cases, we can fix a partition of $\mathcal{V}$ into a set of black vertices $\mathcal{B}$ and white vertices $\mathcal{W}$, such that edges only connect black and white vertices together. We also set $G^{\mathcal{B}}$ (resp. $G^{\mathcal{W}}$ ) to be the graph formed by black (resp. white) vertices, joined iff they form the diagonal of a face of $\mathcal{Q}$. Finally, in the toric case, we suppose that there are two simple cycles $\gamma_{x}^{\mathcal{B}}$ and $\gamma_{y}^{\mathcal{B}}$ on $G^{\mathcal{B}}$ that wind once, respectively horizontally and vertically on the torus; see Fig. 12 .

The dual of $\mathcal{Q}$, denoted by $\mathcal{Q}^{*}$, is the embedded graph whose set of vertices is $\mathcal{F}$ and which has edges connecting elements of $\mathcal{F}$ that are adjacent in $\mathcal{Q}$. We denote by $\mathcal{E}^{*}$ the set of edges of $\mathcal{Q}^{*}$; for an edge $e \in \mathcal{E}$, we denote by $e^{*}$ its corresponding dual edge.

2.1. Eight-vertex-model. An $8 \mathrm{~V}$-configuration is a subset $\tau \subset \mathcal{E}^{*}$ such that, at each face $f \in \mathcal{F}$, an even number of dual edges that belong to $\tau$ meet at $f$. Thus at any face $f \in \mathcal{F}, \tau$ has to be one of the eight types shown in Fig. 4. Let $\Omega(\mathcal{Q})$ be the set of all $8 \mathrm{~V}$-configurations on $\mathcal{Q}$.

Let $A, B, C, D$ be four functions from $\mathcal{F}$ to $\mathbb{R}$. We associate to $f$ a local weight function $w_{f}$, such that $w_{f}(\tau)$ is either $A(f), B(f), C(f)$ or $D(f)$ depending on the local configuration, as in Fig. 4. In the spherical and toric cases, the global weight of $\tau$ is defined as

$$
w_{8 V}(\tau)=\prod_{f \in \mathcal{F}} w_{f}(\tau)
$$

It is more difficult to make sense of formula (5) in the planar case, where the product becomes infinite, requiring the construction of an appropriate Gibbs measure. We discuss this construction in the case of a $Z$-invariant, free-fermion model in Sect. 5.4.

For the remainder of this section, we only deal with the spherical and toric cases. Let $X=(A, B, C, D)$ denote the whole set of weights. The partition function of the model is

$$
\mathcal{Z}_{8 V}=\mathcal{Z}_{8 V}(\mathcal{Q}, X)=\sum_{\tau \in \Omega(\mathcal{Q})} w_{8 V}(\tau)
$$



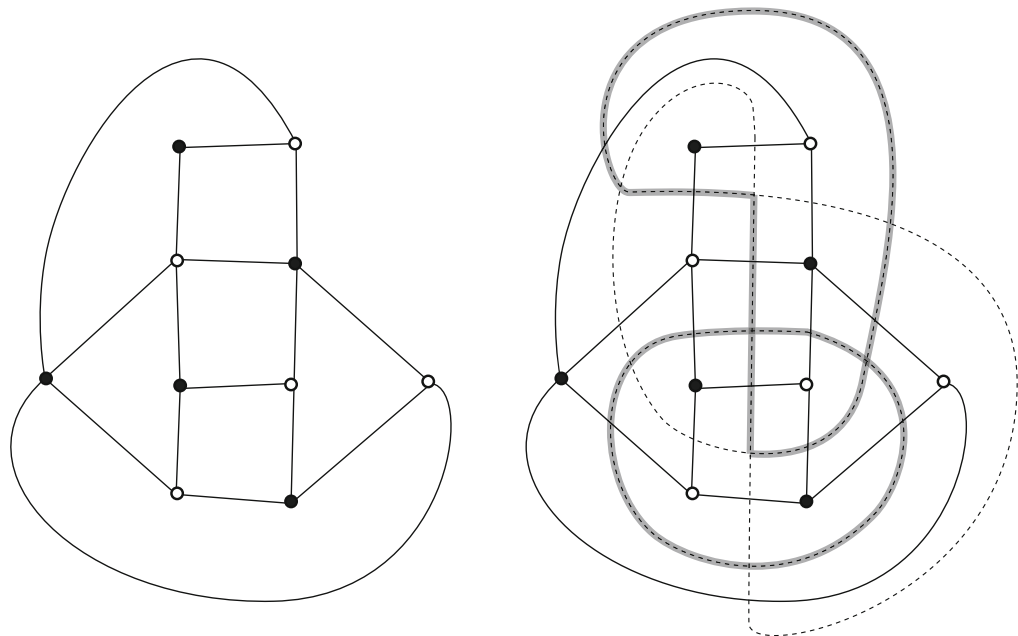

Fig. 5. Left: a quadrangulation $\mathcal{Q}$ in the spherical case. Right: the same quadrangulation with its dual $\mathcal{Q}^{*}$ (dashed) and an eight-vertex configuration

When the weights $X$ take values in positive real numbers, the Boltzmann measure associated to $X$ is the probability measure on $\Omega(\mathcal{Q})$ defined by

$$
\mathbb{P}_{8 V}(\tau)=\frac{w(\tau)}{\mathcal{Z}_{8 V}}
$$

Even if we are only interested in the positive real values in fine, it is convenient to let the weights $X$ take any real values. In this case, $w(\tau)$ and $\mathcal{Z}_{8 V}$ are still well-defined but (6) does not define a probability measure.

A gauge transformation at some face $f \in \mathcal{F}$ consists in multiplying the weights $A(f), B(f), C(f), D(f)$ by the same constant $\lambda \neq 0$. This has the effect of multiplying all weights $w_{8 V}(\tau)$ by $\lambda$, and $\mathcal{Z}_{8 V}$ is also multiplied by $\lambda$. Thus the Boltzmann measure is unchanged.

The weights $X=(A, B, C, D)$ are said to be free-fermion if

$$
\forall f \in \mathcal{F}, A(f)^{2}+B(f)^{2}=C(f)^{2}+D(f)^{2} .
$$

They are said to be standard if

$$
\forall f \in \mathcal{F}, C(f) \neq 0 .
$$

Lemma 7. Let $a, b, c, d$ be real numbers such that $c \neq 0$ and

$$
a^{2}+b^{2}=c^{2}+d^{2} \text {. }
$$

Then there exists a couple $(\alpha, \beta) \in(\mathbb{R} / 2 \pi \mathbb{Z})^{2}$ such that in homogeneous coordinates,

$$
\begin{aligned}
{[a: b: c: d] } & =\left[\begin{array}{c}
\sin \alpha+\sin \beta: \\
\cos \alpha+\cos \beta: \\
1+\sin \alpha \sin \beta+\cos \alpha \cos \beta: \\
\cos \alpha \sin \beta-\sin \alpha \cos \beta
\end{array}\right] \\
& =\left[\sin \left(\frac{\alpha+\beta}{2}\right): \cos \left(\frac{\alpha+\beta}{2}\right): \cos \left(\frac{\alpha-\beta}{2}\right): \sin \left(\frac{-\alpha+\beta}{2}\right)\right] .
\end{aligned}
$$


Proof. We can rewrite (7) as

$$
\left(\frac{a}{\lambda}\right)^{2}+\left(\frac{b}{\lambda}\right)^{2}=\left(\frac{c}{\lambda}\right)^{2}+\left(\frac{d}{\lambda}\right)^{2}=1
$$

for some constant $\lambda>0$. Thus there exists $u, v \in \mathbb{R} / 2 \pi \mathbb{Z}$ such that

$$
[a: b: c: d]=[\sin u: \cos u: \cos v:-\sin v] .
$$

Then we define $\alpha=u+v, \beta=u-v$, which gives the form (9) of the homogeneous coordinates. The form (8) is obtained by multiplying all weights of (9) by $2 \cos \left(\frac{\alpha-\beta}{2}\right)$, which is non zero because $c \neq 0$, and performing simple trigonometric computations.

For that reason, we fix two functions $\alpha, \beta: \mathcal{F} \rightarrow \mathbb{R} / 2 \pi \mathbb{Z}$ and we define the associated free-fermion weights by the following formula, implicitly evaluated at any $f \in \mathcal{F}$ :

$$
X_{\alpha, \beta}=\left(\begin{array}{c}
A_{\alpha, \beta} \\
B_{\alpha, \beta} \\
C_{\alpha, \beta} \\
D_{\alpha, \beta}
\end{array}\right)=\left(\begin{array}{c}
\sin \alpha+\sin \beta \\
\cos \alpha+\cos \beta \\
1+\sin \alpha \sin \beta+\cos \alpha \cos \beta \\
\cos \alpha \sin \beta-\sin \alpha \cos \beta
\end{array}\right) .
$$

By Lemma 7, any standard free-fermion $8 \mathrm{~V}$-model can be written in this way, after proper gauge transformations.

Remark 8.

- The weights $X_{\alpha, \beta}$ satisfy

$$
2 C_{\alpha, \beta}=A_{\alpha, \beta}^{2}+B_{\alpha, \beta}^{2}=C_{\alpha, \beta}^{2}+D_{\alpha, \beta}^{2} .
$$

As a result, given standard free-fermion $8 \mathrm{~V}$-weights $X=(A, B, C, D)$, one gets to the weights $X_{\alpha, \beta}$ by applying gauge transformations at each face with parameter $\lambda(f)=\frac{2 C(f)}{A(f)^{2}+B(f)^{2}}$.

- The weight $X_{\alpha, \beta}$ are standard iff

$$
\forall f \in \mathcal{F}, \alpha(f)-\beta(f) \neq \pi[2 \pi] .
$$

We also say that $\alpha, \beta$ are standard when (12) is satisfied. Note that if this is not the case at some face $f \in \mathcal{F}$, then all the weights $A(f), B(f), C(f), D(f)$ vanish.

- If $\alpha, \beta$ lie in the range

$$
\forall f \in \mathcal{F}, 0<\alpha(f) \leq \beta(f)<\frac{\pi}{2}
$$

then the weights $A_{\alpha, \beta}, B_{\alpha, \beta}, C_{\alpha, \beta}$ are positive and $D_{\alpha, \beta}$ is non-negative. As a result, the Boltzmann measure is a probability measure.

- If $\alpha=\beta$, then the weights $D_{\alpha, \beta}$ vanish and we are left with a $6 \mathrm{~V}$ model. We simply denote $X_{\alpha}$ the weights in that case, and $\mathcal{Z}_{6 V}\left(\mathcal{Q}, X_{\alpha}\right)$ for the partition function. We have

$$
\left[A_{\alpha}: B_{\alpha}: C_{\alpha}\right]=[\sin \alpha: \cos \alpha: 1] .
$$

- Switching $\alpha$ and $\beta$ has the effect of multiplying the weights $D$ by -1 . Since any $8 \mathrm{~V}$-configuration contains an even number of $D$ faces (see [26]), in both the spherical and toric cases,

$$
\mathcal{Z}_{8 V}\left(\mathcal{Q}, X_{\alpha, \beta}\right)=\mathcal{Z}_{8 V}\left(\mathcal{Q}, X_{\beta, \alpha}\right)
$$


2.2. Ising model. Let $\alpha, \beta: \mathcal{F} \rightarrow\left(0, \frac{\pi}{2}\right)$. Let $J_{\mathcal{B}}^{\alpha}, J_{\mathcal{W}}^{\beta}: \mathcal{F} \rightarrow \mathbb{R}$ be the following coupling constants:

$$
\forall f \in \mathcal{F}, \quad J_{\mathcal{B}}^{\alpha}(f)=\frac{1}{2} \ln \left(\frac{1+\sin \alpha(f)}{\cos \alpha(f)}\right), \quad J_{\mathcal{W}}^{\beta}(f)=\frac{1}{2} \ln \left(\frac{1+\cos \beta(f)}{\sin \beta(f)}\right)
$$

An spin configuration on $G^{\mathcal{B}}$ (resp. $G^{\mathcal{W}}$ ) is an application from $\mathcal{B}$ (resp. $\left.\mathcal{W}\right)$ to $\{ \pm 1\}$. The weight of such a configuration $\sigma_{\mathcal{B}}\left(\right.$ resp. $\left.\sigma_{\mathcal{W}}\right)$ is defined as

$$
w_{\mathcal{B}}\left(\sigma_{\mathcal{B}}\right)=\prod_{f \in \mathcal{F}} e^{J_{\mathcal{B}}^{\alpha}(f) \sigma_{u} \sigma_{v}}, \quad w_{\mathcal{W}}\left(\sigma_{\mathcal{W}}\right)=\prod_{f \in \mathcal{F}} e^{J_{\mathcal{W}}^{\beta}(f) \sigma_{x} \sigma_{y}}
$$

where $u, v$ are the black vertices of $f$, and $x, y$ its white vertices.

The partition functions are:

$$
\mathcal{Z}_{\text {Ising }}^{\mathcal{B}}=\mathcal{Z}_{\text {Ising }}^{\mathcal{B}}\left(J_{\mathcal{B}}^{\alpha}\right)=\sum_{\sigma_{\mathcal{B}}} w_{\mathcal{B}}\left(\sigma_{\mathcal{B}}\right), \quad \mathcal{Z}_{\text {Ising }}^{\mathcal{W}}=\mathcal{Z}_{\text {Ising }}^{\mathcal{W}}\left(J_{\mathcal{W}}^{\beta}\right)=\sum_{\sigma_{\mathcal{W}}} w_{\mathcal{W}}\left(\sigma_{\mathcal{W}}\right)
$$

where the sums are over spin configurations. Again, the associated Boltzmann measure is

$$
\mathbb{P}_{\text {Ising }}^{\mathcal{B}}\left(\sigma_{\mathcal{B}}\right)=\frac{w_{\mathcal{B}}\left(\sigma_{\mathcal{B}}\right)}{\mathcal{Z}_{\text {Ising }}^{\mathcal{B}}}, \quad \mathbb{P}_{\text {Ising }}^{\mathcal{W}}\left(\sigma_{\mathcal{W}}\right)=\frac{w_{\mathcal{W}}\left(\sigma_{\mathcal{W}}\right)}{\mathcal{Z}_{\text {Ising }}^{\mathcal{W}}}
$$

2.3. Dimer model. Let $G=(V, E)$ be a finite graph, equipped with real weights on the edges $\left(v_{e}\right)_{e \in E}$. A dimer configuration, or perfect matching, is a subset of edges $m \subset E$ such that every vertex of $G$ is adjacent to exactly one edge of $m$. We denote by $\mathcal{M}(G)$ the set of all dimer configurations on $G$.

The Boltzmann measure on $\mathcal{M}(G)$ is defined by

$$
\mathbb{P}_{\operatorname{dim}}(m)=\frac{\prod_{e \in m} v_{e}}{\mathcal{Z}_{\operatorname{dim}}(G, v)}
$$

where $\mathcal{Z}_{\operatorname{dim}}(G, v)$ is the partition function:

$$
\mathcal{Z}_{\operatorname{dim}}(G, v)=\sum_{m \in \mathcal{M}(G)} \prod_{e \in m} v_{e}
$$

2.4. Order and disorder variables. The notions of order and disorder variables were defined by Kadanoff and Ceva for the Ising model [40] and play a central role in the study of spinor and fermionic observables; see for instance [18] for a unifying review. The definition for the Ising model is classical; for the $8 \mathrm{~V}$ model, we adapt definitions of Dubédat [26].

For these definitions $\mathcal{Q}$ can be a quadrangulation in the spherical or toric case. 
2.4.1. Ising correlators Let $B_{0} \subset \mathcal{B}$ and $W_{1} \subset \mathcal{W}$ be two subset of black and white vertices of $\mathcal{Q}$, of even cardinality. Let $\gamma_{B_{0}}$ be the union of disjoint simple paths on $G^{\mathcal{B}}$ that connect the vertices of $B_{0}$ pairwise (these are called order lines); $\gamma_{B_{0}}$ can be alternatively seen as a subset of $\mathcal{F}$. We similarly define $\gamma_{W_{1}}$ as a union of disjoint simple paths on $G^{\mathcal{W}}$ that connect the $W_{1}$ pairwise (also called disorder lines).

Let $\alpha: \mathcal{F} \rightarrow\left(0, \frac{\pi}{2}\right)$. We modify the coupling constants $J_{\mathcal{B}}^{\alpha}$ by adding $i \frac{\pi}{2}$ to the coupling constant at $f$ when $f \in \gamma_{B_{0}}$, and afterwards multiplying the coupling constant by -1 when $f \in \gamma_{W_{1}}$ (the order is important). Let $J^{\prime}$ be these new coupling constants. Then the mixed correlator of Kadanoff and Ceva is defined as

$$
\left\langle\prod_{b \in B_{0}} \sigma_{b} \prod_{w \in W_{1}} \mu_{w}\right\rangle_{\alpha, \gamma_{B_{0}}, \gamma_{W_{1}}}^{\operatorname{Ising} \mathcal{B}}=\left\langle\sigma\left(B_{0}\right) \mu\left(W_{1}\right)\right\rangle_{\alpha, \gamma_{B_{0}}, \gamma_{W_{1}}}^{\operatorname{Ising} \mathcal{B}}:=\mathcal{Z}_{\text {Ising }}^{\mathcal{B}}\left(J^{\prime}\right) .
$$

This depends on the choice of paths and the order of operations on the coupling constants, but only up to a global sign. The order variables are simply the spins $\sigma_{b}$, with $\langle\cdot\rangle$ representing an unnormalized expectation under the Boltzmann measure. The disorder variables $\mu_{w}$ represent defects in the configuration, and are conjugated with order variables under Kramers-Wannier duality [51]. Again we refer to [40].

Similarly for the Ising model on $G^{\mathcal{W}}$, if $W_{0} \subset \mathcal{W}$ and $B_{1} \subset \mathcal{B}$ are even subsets, we chose paths $\gamma_{W_{0}}, \gamma_{B_{1}}$. Then for $\beta: \mathcal{F} \rightarrow\left(0, \frac{\pi}{2}\right)$, we add $i \frac{\pi}{2}$ to the constants $J_{\beta}^{\mathcal{W}}$ on $\gamma_{W_{0}}$, then multiply the constants by -1 on $\gamma_{B_{1}}$, and we name the new constants $J^{\prime \prime}$. The mixed correlator is

$$
\left\langle\prod_{w \in W_{0}} \sigma_{w} \prod_{b \in B_{1}} \mu_{b}\right\rangle_{\beta, \gamma_{W_{0}}, \gamma_{B_{1}}}^{\operatorname{Ising} \mathcal{W}}=\left\langle\sigma\left(W_{0}\right) \mu\left(B_{1}\right)\right\rangle_{\beta, \gamma_{W_{0}}, \gamma_{B_{1}}}^{\operatorname{Ising} \mathcal{W}}:=\mathcal{Z}_{\text {Ising }}^{\mathcal{W}}\left(J^{\prime \prime}\right)
$$

2.4.2. 8 V correlators Order and disorder variables for the $8 \mathrm{~V}$-model are defined in [26]. The following definition is original but it is easy to check that it is equivalent to that of [26]. In the $8 \mathrm{~V}$ case, order and disorder variables can be located on either black or white vertices of $\mathcal{Q}$.

Definition 9. Let $\mathcal{Q}$ be a quadrangulation in the spherical case. Let $X=(A, B, C, D)$ : $\mathcal{F} \rightarrow \mathbb{R}^{4}$ be a family of weights. For every $f \in \mathcal{F}$, we define operators $\nu_{f}^{\mathcal{B}}, v_{f}^{\mathcal{W}}, \xi_{f}^{\mathcal{B}}, \xi_{f}^{\mathcal{W}}$ that act on $X$ by transforming $X(f)$ in the following way:

$$
\begin{aligned}
\nu_{f}^{\mathcal{B}}:\left(\begin{array}{l}
A(f) \\
B(f) \\
C(f) \\
D(f)
\end{array}\right) \mapsto\left(\begin{array}{c}
A(f) \\
-B(f) \\
C(f) \\
-D(f)
\end{array}\right) & \nu_{f}^{\mathcal{W}}:\left(\begin{array}{l}
A(f) \\
B(f) \\
C(f) \\
D(f)
\end{array}\right) \mapsto\left(\begin{array}{c}
-A(f) \\
B(f) \\
C(f) \\
-D(f)
\end{array}\right) \\
\xi_{f}^{\mathcal{B}}:\left(\begin{array}{l}
A(f) \\
B(f) \\
C(f) \\
D(f)
\end{array}\right) \mapsto\left(\begin{array}{c}
C(f) \\
D(f) \\
A(f) \\
B(f)
\end{array}\right) & \xi_{f}^{\mathcal{W}}:\left(\begin{array}{l}
A(f) \\
B(f) \\
C(f) \\
D(f)
\end{array}\right) \mapsto\left(\begin{array}{c}
D(f) \\
C(f) \\
B(f) \\
A(f)
\end{array}\right)
\end{aligned}
$$

Let $B_{0}, B_{1} \subset \mathcal{B}$ (resp. $W_{0}, W_{1} \subset \mathcal{W}$ ) be two even subsets of black (resp. white) vertices, with simple paths $\gamma_{B_{0}}, \gamma_{B_{1}}$ (resp. $\gamma_{W_{0}}, \gamma_{W_{1}}$ ) joining them pairwise. As these paths use black (resp. white) diagonals of faces, we can identify them with subsets of $\mathcal{F}$. 
Let $\gamma=\left(\gamma_{B}, \gamma_{W}, \gamma_{B^{\prime}}, \gamma_{W^{\prime}}\right)$. We define modified weights $X_{\gamma}^{\prime}$ obtained by the following composition of operators:

$$
X_{\gamma}^{\prime}=\left(\prod_{f \in \gamma_{B}} \xi_{f}^{\mathcal{B}} \prod_{f \in \gamma_{W_{1}}} \xi_{f}^{\mathcal{W}} \prod_{f \in \gamma_{B_{0}}} v_{f}^{\mathcal{B}} \prod_{f \in \gamma_{W_{0}}} v_{f}^{\mathcal{W}}\right) X
$$

We define the mixed correlator as:

$$
\left\langle\prod_{b \in B_{0}} \sigma_{b} \prod_{w \in W_{0}} \sigma_{w} \prod_{b \in B_{1}} \mu_{b} \prod_{w \in W_{1}} \mu_{w}\right\rangle_{X, \gamma}^{8 V}:=\mathcal{Z}_{8 V}\left(X_{\gamma}^{\prime}\right) .
$$

We will also use the shorthand notation $\left\langle\sigma\left(B_{0}\right) \sigma\left(W_{0}\right) \mu\left(B_{1}\right) \mu\left(W_{1}\right)\right\rangle_{X, \gamma}^{8 V}$.

Remark 10.

- Mixed correlators may depend on the set of paths $\gamma$, but only up to a global sign [26]. Also note that in (15) we always apply order operators before disorder ones. This is important because these operators do not always commute. The only cases were they do not commute are

$$
\begin{aligned}
& v_{f}^{\mathcal{B}} \xi_{f}^{\mathcal{W}}=-\xi_{f}^{\mathcal{W}} v_{f}^{\mathcal{B}}, \\
& v_{f}^{\mathcal{W}} \xi_{f}^{\mathcal{B}}=-\xi_{f}^{\mathcal{B}} \nu_{f}^{\mathcal{W}},
\end{aligned}
$$

but like for path dependence, changing the order might only multiply the correlator by a factor -1 .

- Again these correlators can be interpreted as unnormalized expectations under the Boltzmann measure. If $b, b^{\prime} \in B_{0}$, then the couple of order variables $\sigma_{b} \sigma_{b^{\prime}}$ represents the random variable $(-1)^{n}$ where $n$ is the number of edges in the $8 \mathrm{~V}$ configuration on any path on $\mathcal{Q}$ between $b$ and $b^{\prime}$.

The disorder variables are equivalent to modifying every eight-vertex configuration by applying a XOR with the configuration of "half edges" shown in Fig. 6. The resulting "configuration" is no longer a subset of edges of $\mathcal{Q}$, but we could still define its weight as in (5). The advantage of modifying weights with the operators of Definition 9 is that we never actually have to work with these disordered configurations.

\section{Couplings of 8V-Models}

We review classical results on the Ising-8V correspondence [5], on the $8 \mathrm{~V}$ duality [68], in terms of order and disorder variables [26]. We then apply them to prove couplings results for different free-fermion $8 \mathrm{~V}$-models.

3.1. Spin-vertex correspondence. The spin-vertex correspondence comes from the following simple remark, that seems to be due to Baxter [5]. If we superimpose two spin configurations, one on $G^{\mathcal{B}}$ and one on $G^{\mathcal{W}}$, and we draw the interfaces between +1 and -1 spins, we get an $8 \mathrm{~V}$-configuration. This transformation is two-to-one, and can be made weight-preserving by choosing the appropriate $8 \mathrm{~V}$ weights. 


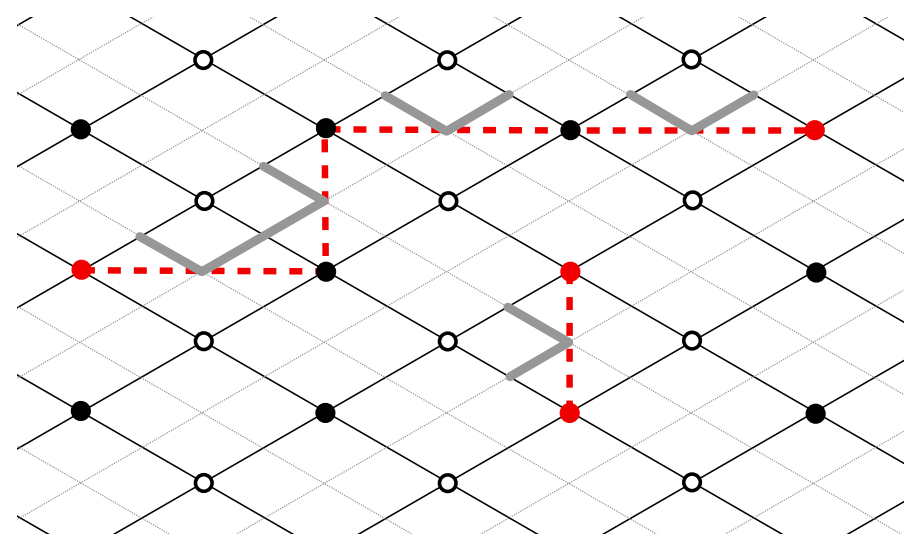

Fig. 6. A piece of a quadrangulation, a subset $B_{1} \subset \mathcal{B}$ with paths $\gamma_{B_{1}}$ joining them pairwise (dashed), and a partial configuration (bold grey)

Proposition 11 ([5,26]). Let $\mathcal{Q}$ be a quadrangulation in the spherical case, and $\alpha, \beta$ : $\mathcal{F} \rightarrow\left(0, \frac{\pi}{2}\right)$. Consider the $8 V$-weights $X: \mathcal{F} \rightarrow \mathbb{R}^{4}$ given by

$$
X=\left(\begin{array}{c}
e^{J_{\mathcal{B}}^{\alpha}-J_{\mathcal{W}}^{\beta}} \\
e^{-J_{\mathcal{B}}^{\alpha}+J_{\mathcal{W}}^{\beta}} \\
e^{J_{\mathcal{B}}^{\alpha}+J_{\mathcal{W}}^{\beta}} \\
e^{-J_{\mathcal{B}}^{\alpha}-J_{\mathcal{W}}^{\beta}}
\end{array}\right) .
$$

Then for any $B_{0}, B_{1}, W_{0}, W_{1}$ and paths $\gamma$ as in Definition 9,

$2\left\langle\sigma\left(B_{0}\right) \sigma\left(W_{0}\right) \mu\left(B_{1}\right) \mu\left(W_{1}\right)\right\rangle_{X, \gamma}^{8 V}=\left\langle\sigma\left(B_{0}\right) \mu\left(W_{1}\right)\right\rangle_{\alpha, \gamma_{B_{0}}, \gamma_{W_{1}}}^{I \operatorname{sing} \mathcal{B}}\left\langle\sigma\left(W_{0}\right) \mu\left(B_{1}\right)\right\rangle_{\beta, \gamma_{W_{0}}, \gamma_{B_{1}}}^{I \operatorname{sing} \mathcal{W}}$. In particular,

$$
2 \mathcal{Z}_{8 V}(\mathcal{Q}, X)=\mathcal{Z}_{\text {Ising }}^{\mathcal{B}}\left(J_{\mathcal{B}}^{\alpha}\right) \times \mathcal{Z}_{\text {Ising }}^{\mathcal{W}}\left(J_{\mathcal{W}}^{\beta}\right)
$$

3.2. Modifications of weights. One key feature of the $8 \mathrm{~V}$-model is its duality relation. This is an instance of Kramers-Wannier duality [51], and in the case of the eight-vertex model it was discovered by $\mathrm{Wu}$ [68] using high-temperature expansion techniques. The formulation for correlators comes from Dubédat [26], and means that duality exchanges order and disorder. We give an interpretation in terms of discrete Fourier transform in Appendix A.

Proposition 12 ([26,68]). Let $\mathcal{Q}$ be a quadrangulation in the spherical case, and let $X=(A, B, C, D): \mathcal{F} \rightarrow \mathbb{R}^{4}$ be any $8 V$-weights. Let $\hat{X}=(\hat{A}, \hat{B}, \hat{C}, \hat{D})$ be defined by

$$
\forall f \in \mathcal{F},\left(\begin{array}{l}
\hat{A}(f) \\
\hat{B}(f) \\
\hat{C}(f) \\
\hat{D}(f)
\end{array}\right)=\frac{1}{2}\left(\begin{array}{cccc}
1 & -1 & 1 & -1 \\
-1 & 1 & 1 & -1 \\
1 & 1 & 1 & 1 \\
-1 & -1 & 1 & 1
\end{array}\right)\left(\begin{array}{l}
A(f) \\
B(f) \\
C(f) \\
D(f)
\end{array}\right) .
$$


Then for any $B_{0}, B_{1}, W_{0}, W_{1}$ and paths $\gamma$ as in Definition 9 , let $\hat{\gamma}=\left(\gamma_{B_{1}}, \gamma_{W_{1}}, \gamma_{B_{0}}, \gamma_{W_{0}}\right)$; we have

$$
\left\langle\sigma\left(B_{0}\right) \sigma\left(W_{0}\right) \mu\left(B_{1}\right) \mu\left(W_{1}\right)\right\rangle_{X, \gamma}^{8 V}=\left\langle\sigma\left(B_{1}\right) \sigma\left(W_{1}\right) \mu\left(B_{0}\right) \mu\left(W_{0}\right)\right\rangle_{\hat{X}, \hat{\gamma}}^{8 V} .
$$

In particular,

$$
\mathcal{Z}_{8 V}(\mathcal{Q}, X)=\mathcal{Z}_{8 V}(\mathcal{Q}, \hat{X})
$$

Another transformation of weights consists in multiplying all weights $D(f)$ by -1 . As any $8 \mathrm{~V}$-configuration contains an even number of faces of type $D$, this does not change its global weight. However, in correlators containing disorder operators, the effect is non trivial; a result of [26] is that $\mu$ variables become $\sigma \mu$, while $\sigma$ variables are unchanged, we rephrase it here using symmetric differences $\triangle$.

Proposition 13 ([26]). Let $\mathcal{Q}$ be a quadrangulation in the spherical case, and let $X=$ $(A, B, C, D): \mathcal{F} \rightarrow \mathbb{R}^{4}$ be any $8 V$-weights. Let $X^{\prime}=(A, B, C,-D)$.

Then for any $B_{0}, B_{1}, W_{0}, W_{1}$ and paths $\gamma$ as in Definition 9, let $\gamma^{\prime}=$ $\left(\gamma_{B_{0}} \Delta \gamma_{B_{1}}, \gamma_{W_{0}} \Delta \gamma_{W_{1}}, \gamma_{B_{1}}, \gamma_{W_{1}}\right)$; we have

$$
\left\langle\sigma\left(B_{0}\right) \sigma\left(W_{0}\right) \mu\left(B_{1}\right) \mu\left(W_{1}\right)\right\rangle_{X, \gamma}^{8 V}=\left\langle\sigma\left(B_{0} \Delta B_{1}\right) \sigma\left(W_{0} \Delta W_{1}\right) \mu\left(B_{1}\right) \mu\left(W_{1}\right)\right\rangle_{X^{\prime}, \gamma^{\prime}}^{8 V} .
$$

In particular,

$$
\mathcal{Z}_{8 V}(\mathcal{Q}, X)=\mathcal{Z}_{8 V}\left(\mathcal{Q}, X^{\prime}\right)
$$

3.3. Free-fermion $8 \mathrm{~V}$ correlators. By combining the previous results, we can relate free-fermion $8 \mathrm{~V}$ correlations with Ising ones. This has been done in [26] when the Ising models are dual of each other (which in our case corresponds to $\alpha=\beta$ ), but the proof works identically when this is not the case.

Corollary 14. Let $\mathcal{Q}$ be a quadrangulation in the spherical case, and let $\alpha, \beta: \mathcal{F} \rightarrow$ $\left(0, \frac{\pi}{2}\right)$.

For any $B_{0}, B_{1}, W_{0}, W_{1}$ and paths $\gamma$ as in Definition 9 , let $\gamma_{\mathcal{B}}=\left(\gamma_{B_{0}}, \gamma_{W_{0}} \Delta \gamma_{W_{1}}\right)$ and $\gamma \mathcal{W}=\left(\gamma_{W_{0}}, \gamma_{B_{0}} \Delta \gamma_{B_{1}}\right)$. Then

$$
\begin{aligned}
& \left\langle\sigma\left(B_{0}\right) \sigma\left(W_{0}\right) \mu\left(B_{1}\right) \mu\left(W_{1}\right)\right\rangle_{X_{\alpha, \beta}, \gamma}^{8 V}=c_{0}\left\langle\sigma\left(B_{0}\right) \mu\left(W_{0} \triangle W_{1}\right)\right\rangle_{\alpha, \gamma_{\mathcal{B}}}^{I \text { sing } \mathcal{B}} \\
& \quad\left\langle\sigma\left(W_{0}\right) \mu\left(B_{0} \triangle B_{1}\right)\right\rangle_{\beta, \gamma \mathcal{W}}^{I s i n g \mathcal{W}}
\end{aligned}
$$

where

$$
c_{0}=\frac{1}{2} \prod_{f \in \mathcal{F}} \sqrt{\cos \alpha(f) \sin \beta(f) C_{\alpha, \beta}(f)} .
$$

In particular,

$$
\mathcal{Z}_{8 V}\left(\mathcal{Q}, X_{\alpha, \beta}\right)=c_{0} \mathcal{Z}_{\text {Ising }}^{\mathcal{B}}\left(J_{\mathcal{B}}^{\alpha}\right) \mathcal{Z}_{\text {Ising }}^{\mathcal{W}}\left(J_{\mathcal{W}}^{\beta}\right)
$$


Proof. From Proposition 11, the product of Ising correlators on the right-hand side of (19) is equal to

$$
2\left\langle\sigma\left(B_{0}\right) \sigma\left(W_{0}\right) \mu\left(B_{0} \Delta B_{1}\right) \mu\left(W_{0} \Delta W_{1}\right)\right\rangle_{X,\left(\gamma_{\mathcal{B}}, \gamma_{\mathcal{W}}\right)}^{8 V}
$$

for the weights $X=(A, B, C, D)$ given by

$$
\left\{\begin{array}{l}
A=e^{J_{\mathcal{B}}^{\alpha}-J_{\mathcal{W}}^{\beta}}=\sqrt{\frac{1+\sin \alpha}{\cos \alpha} \frac{\sin \beta}{1+\cos \beta}}, \\
B=e^{-J_{\mathcal{B}}^{\alpha}+J_{\mathcal{W}}^{\beta}}=\sqrt{\frac{\cos \alpha}{1+\sin \alpha} \frac{1+\cos \beta}{\sin \beta}} \\
C=e^{J_{\mathcal{B}}^{\alpha}+J_{\mathcal{W}}^{\beta}}=\sqrt{\frac{1+\sin \alpha}{\cos \alpha} \frac{1+\cos \beta}{\sin \beta}}, \\
D=e^{-J_{\mathcal{B}}^{\alpha}-J_{\mathcal{W}}^{\beta}}=\sqrt{\frac{\cos \alpha}{1+\sin \alpha} \frac{\sin \beta}{1+\cos \beta}} .
\end{array}\right.
$$

On these weights, we perform the transformation of Proposition 13, then of Proposition 12, and again of Proposition 13. This amounts to defining $\tilde{X}=(\tilde{A}, \tilde{B}, \tilde{C}, \tilde{D})$ by

$$
\left(\begin{array}{c}
\tilde{A} \\
\tilde{B} \\
\tilde{C} \\
\tilde{D}
\end{array}\right)=\frac{1}{2}\left(\begin{array}{cccc}
1 & -1 & 1 & 1 \\
-1 & 1 & 1 & 1 \\
1 & 1 & 1 & -1 \\
1 & 1 & -1 & 1
\end{array}\right)\left(\begin{array}{l}
A \\
B \\
C \\
D
\end{array}\right)
$$

Following the transformations in the Propositions, we get that the Ising correlators are equal to

$$
2\left\langle\sigma\left(B_{0}\right) \sigma\left(W_{0}\right) \mu\left(B_{1}\right) \mu\left(W_{1}\right)\right\rangle_{\tilde{X}, \gamma}^{8 V} .
$$

Trigonometric computations show that (implicitly at any $f \in \mathcal{F}$ ):

$$
\left(\begin{array}{c}
\tilde{A} \\
\tilde{B} \\
\tilde{C} \\
\tilde{D}
\end{array}\right)=\frac{1}{\sqrt{\cos \alpha \sin \beta(1+\cos (\alpha-\beta))}}\left(\begin{array}{c}
A_{\alpha, \beta} \\
B_{\alpha, \beta} \\
C_{\alpha, \beta} \\
D_{\alpha, \beta}
\end{array}\right)
$$

and using the definition of correlators as partition function, we see that these gauge transformations multiply the correlator by the same factor.

3.4. Coupling of free-fermion $8 V$-models. With Corollary 14 , we are able to factor correlators for the weights $X_{\alpha, \beta}$ into a part that depends on $\alpha$ and a part that depends on $\beta$. By doing the same for $X_{\alpha^{\prime}, \beta^{\prime}}$ and rearranging the Ising correlators, we can get the correlators of $X_{\alpha, \beta^{\prime}}$ and $X_{\alpha^{\prime}, \beta}$. This is expressed in the following "switching" result. The constants can be defined in terms of

$$
c_{\alpha, \beta}=\prod_{f \in \mathcal{F}} C_{\alpha, \beta}(f)=\prod_{f \in \mathcal{F}} \frac{A_{\alpha, \beta}^{2}(f)+B_{\alpha, \beta}^{2}(f)}{2} .
$$


Theorem 15. Let $\mathcal{Q}$ be a quadrangulation in the spherical case, and let $\alpha, \beta, \alpha^{\prime}, \beta^{\prime}$ : $\mathcal{F} \rightarrow\left(0, \frac{\pi}{2}\right)$. Let $B_{0}, B_{1}, W_{0}, W_{1}, \gamma$ (resp. $\left.B_{0}^{\prime}, B_{1}^{\prime}, W_{0}^{\prime}, W_{1}^{\prime}, \gamma^{\prime}\right)$ be as in Definition 9. Then

$$
\begin{aligned}
& \left\langle\sigma\left(B_{0}\right) \sigma\left(W_{0}\right) \mu\left(B_{1}\right) \mu\left(W_{1}\right)\right\rangle_{X_{\alpha, \beta}, \gamma}^{8 V}\left\langle\sigma\left(B_{0}^{\prime}\right) \sigma\left(W_{0}^{\prime}\right) \mu\left(B_{1}^{\prime}\right) \mu\left(W_{1}^{\prime}\right)\right\rangle_{X_{\alpha^{\prime}, \beta^{\prime}}, \gamma^{\prime}}^{8 V} \\
& \quad=c_{1}\left\langle\sigma ( B _ { 0 } ^ { \prime \prime } ) \sigma ( W _ { 0 } ^ { \prime \prime } ) \mu ( B _ { 1 } ^ { \prime \prime } ) \mu ( W _ { 1 } ^ { \prime \prime } ) | _ { X _ { \alpha , \beta ^ { \prime } } , \gamma ^ { \prime \prime } } ^ { 8 V } \left\langle\left.\sigma\left(B_{0}^{\prime \prime \prime}\right) \sigma\left(W_{0}^{\prime \prime \prime}\right) \mu\left(B_{1}^{\prime \prime \prime}\right) \mu\left(W_{1}^{\prime \prime \prime}\right)\right|_{X_{\alpha^{\prime}, \beta}, \gamma^{\prime \prime \prime}} ^{8 V}\right.\right.
\end{aligned}
$$

where

$$
\left(\begin{array}{c}
B_{0}^{\prime \prime} \\
W_{0}^{\prime \prime} \\
B_{1}^{\prime \prime} \\
W_{1}^{\prime \prime}
\end{array}\right)=\left(\begin{array}{c}
B_{0} \\
W_{0}^{\prime} \\
B_{0} \triangle B_{0}^{\prime} \triangle B_{1}^{\prime} \\
W_{0} \triangle W_{0}^{\prime} \triangle W_{1}
\end{array}\right), \quad\left(\begin{array}{c}
B_{0}^{\prime \prime \prime} \\
W_{0}^{\prime \prime \prime} \\
B_{1}^{\prime \prime \prime} \\
W_{1}^{\prime \prime \prime}
\end{array}\right)=\left(\begin{array}{c}
B_{0}^{\prime} \\
W_{0} \\
B_{0} \triangle B_{0}^{\prime} \triangle B_{1} \\
W_{0} \triangle W_{0}^{\prime} \triangle W_{1}^{\prime}
\end{array}\right),
$$

with the same formulas for the paths in $\gamma^{\prime \prime}, \gamma^{\prime \prime \prime}$, and

$$
c_{1}=\sqrt{\frac{c_{\alpha, \beta} c_{\alpha^{\prime}, \beta^{\prime}}}{c_{\alpha, \beta^{\prime}} c_{\alpha^{\prime}, \beta}}} .
$$

In particular,

$$
\mathcal{Z}_{8 V}\left(\mathcal{Q}, X_{\alpha, \beta}\right) \mathcal{Z}_{8 V}\left(\mathcal{Q}, X_{\alpha^{\prime}, \beta^{\prime}}\right)=c_{1} \mathcal{Z}_{8 V}\left(\mathcal{Q}, X_{\alpha, \beta^{\prime}}\right) \mathcal{Z}_{8 V}\left(\mathcal{Q}, X_{\alpha^{\prime}, \beta}\right) .
$$

Proof. This immediately comes from writing both the left-hand side and the right-hand side in terms of Ising correlators using Corollary 14, and checking that they are the same.

Example 16. By taking $B_{0}=B_{0}^{\prime}=B$ and $W_{0}=W_{0}^{\prime}=W$ (i.e. the initial order variables being the same), we get the simpler formula

$$
\begin{aligned}
& \left\langle\sigma(B) \sigma(W) \mu\left(B_{1}\right) \mu\left(W_{1}\right)\right\rangle_{X_{\alpha, \beta}, \gamma}^{8 V}\left\langle\left.\sigma(B) \sigma(W) \mu\left(B_{1}^{\prime}\right) \mu\left(W_{1}^{\prime}\right)\right|_{X_{\alpha^{\prime}, \beta^{\prime}}, \gamma^{\prime}} ^{8 V}\right. \\
& \quad=c_{1}\left\langle\sigma ( B ) \sigma ( W ) \mu ( B _ { 1 } ^ { \prime } ) \mu ( W _ { 1 } ) | _ { X _ { \alpha , \beta ^ { \prime } } , \gamma ^ { \prime \prime } } ^ { 8 V } \left\langle\left.\sigma(B) \sigma(W) \mu\left(B_{1}\right) \mu\left(W_{1}^{\prime}\right)\right|_{X_{\alpha^{\prime}, \beta}, \gamma^{\prime \prime \prime}} ^{8 V}\right.\right.
\end{aligned}
$$

This nontrivial equality of correlators (and of partition functions) suggests that there exists a coupling between pairs of $8 \mathrm{~V}$-configurations. Specifically, when $(\alpha, \beta)$ and $\left(\alpha^{\prime}, \beta^{\prime}\right)$ satisfy (13), then the $8 \mathrm{~V}$-weights define a Boltzmann probability; if $\tau_{\alpha, \beta}, \tau_{\alpha^{\prime}, \beta^{\prime}}$ are independent and Boltzmann distributed, we want to couple them with $\tau_{\alpha, \beta^{\prime}}, \tau_{\alpha^{\prime}, \beta}$, while keeping as much information as possible.

This is the content of Theorem 3: it is possible to do so while keeping the XOR of configurations equal (i.e. the XOR of the corresponding sets of dual edges of $\mathcal{Q}$, which is still an 8V-configuration). The proof is a direct consequence of Theorem 15, but requires the introduction of a discrete Fourier transform on the space of $8 \mathrm{~V}$-configurations and is postponed to the end of Appendix A. An extended statement can be formulated for the XOR of configurations with disorder, see Remark 43.

\section{Kasteleyn Matrices}

We review the transformation of free-fermion $8 \mathrm{~V}$-configurations into dimers, and we compute special relations for the corresponding (inverse) Kasteleyn matrices. 


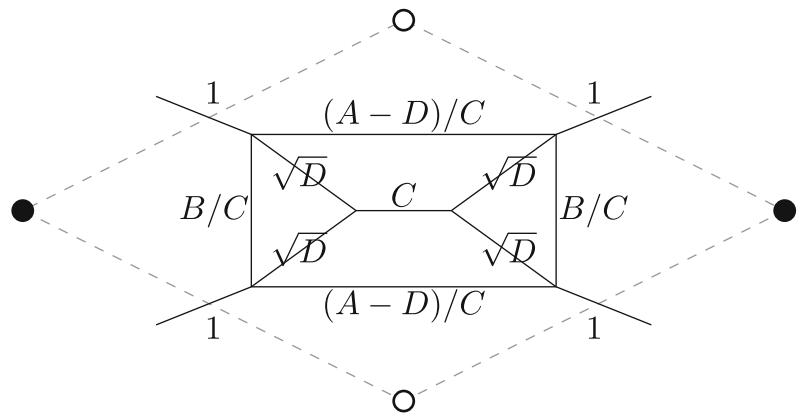

Fig. 7. The quadrangulation $\mathcal{Q}$ (dashed) at a face $f$ and the decorated graph of Fan and Wu [30] (solid lines) with its dimer weights. The functions $A, B, C, D$ are implicitly evaluated at $f$

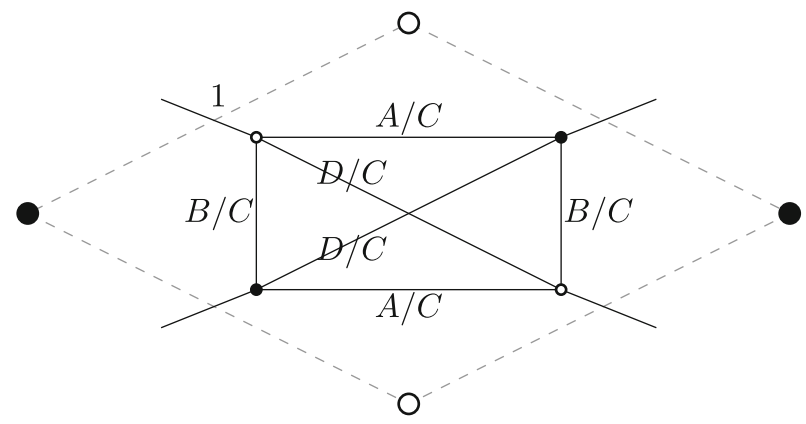

Fig. 8. The decorated graph $G^{T}$ of Hsue et al. [37] with its dimer weights

4.1. Free-fermion $8 \mathrm{~V}$ to dimers. In the case of the square lattice, it has been shown by Fan and Wu that the $8 \mathrm{~V}$-model at its free-fermion point can be transformed into a dimer model on a planar decorated graph [30]. Their arguments work identically on any quadrangulation. The corresponding decorated graph is represented in Fig. 7.

In the more special case of a free-fermionic $6 \mathrm{~V}$ model, the graph becomes bipartite and the dimer model can be studied in more details $[15,26,69]$. No such bipartite dimer decoration is known for the $8 \mathrm{~V}$-model, and the techniques of bipartite dimers are unavailable as such.

In our setting we will make use of another decorated graph due to Hsue, Lin and Wu [37], see Fig. 8. This graph is more symmetric but non planar, which makes the usual theory of dimers as Pfaffians not available, but an adapted theory has been developed by Kasteleyn [42,43].

More precisely, let $G^{T}=\left(V^{T}, E^{T}\right)$ be a decorated version of $\mathcal{Q}^{*}$ obtained by drawing small complete graphs $K_{4}$ inside faces of $\mathcal{Q}$ and joining them by "legs" that cross the edges of $\mathcal{Q}$, as represented in Fig. 8. Even if this graph is not bipartite, we still decompose $V^{T}$ into a subset of black vertices $B^{T}$ and white vertices $W^{T}$, such that the black (resp. white) vertices lie on the left (resp. right) of an edge of $\mathcal{Q}$ oriented from black to white. For every edge e $\in E^{T}$, we define $v_{\mathrm{e}}$ as in Fig. 8. We will need a nonstandard weight for $m \in \mathcal{M}\left(G^{T}\right)$, defined as

$$
\tilde{w}(m)=(-1)^{N(m)} \prod_{\mathrm{e} \in m} v_{\mathrm{e}}
$$


where $N(m)$ is the number of pairs of intersecting edges in $m$. The corresponding partition function is

$$
\tilde{\mathcal{Z}}_{\operatorname{dim}}\left(G^{T}, v\right)=\sum_{m \in \mathcal{M}\left(G^{T}\right)} \tilde{w}(m) .
$$

Note that at the boundary of every face $f \in \mathcal{F}, m$ uses an even number of legs. As a result, if we only keep the occupied legs of $m$, we get an $8 \mathrm{~V}$-configuration $\tau \in \Omega(\mathcal{Q})$. We denote this by $m \mapsto \tau$. This mapping is weight-preserving in the following sense; this was noted when $\mathcal{Q}$ is the square lattice by Hsue, Lin and Wu [37] but works in the exact same way for any quadrangulation:

Theorem 17 ([37]). Let $\mathcal{Q}$ be a quadrangulation in the spherical or toric case, and $X$ a set of standard free-fermionic $8 V$-weights on $\mathcal{Q}$. Then for every $\tau \in \Omega(\mathcal{Q})$,

$$
w_{8 V}(\tau)=\left(\prod_{f \in \mathcal{F}} C(f)\right) \sum_{\substack{m \in \mathcal{M}\left(G^{T}\right) \\ \text { s.t. } m \mapsto \tau}} \tilde{w}(m) .
$$

In particular,

$$
\mathcal{Z}_{8 V}(\mathcal{Q}, X)=\left(\prod_{f \in \mathcal{F}} C(f)\right) \tilde{\mathcal{Z}}_{\operatorname{dim}}\left(G^{T}, v\right)
$$

We now describe how to compute $\tilde{\mathcal{Z}}_{\text {dim }}\left(G^{T}, v\right)$ using an adapted version of Kasteleyn matrices.

4.2. Skew-symmetric real matrix. A Kasteleyn orientation of a planar or toric graph is an orientation of the edges such that every face is clockwise-odd, meaning that it has an odd number of clockwise-oriented edges; by the planarity condition, such an orientation can always be constructed, and may be used to identify the partition function of dimers with the Pfaffian of the corresponding skew-symmetric, weighted adjacency matrix [41,64]. Since $G^{T}$ is non-planar, there might not exist a usual Kasteleyn orientation, but there still exists an admissible orientation so that the Pfaffian is equal to $\tilde{\mathcal{Z}}_{\operatorname{dim}}\left(G^{T}, v\right)[42,43]$, which we describe now.

If we remove all edges of $G^{T}$ that join black vertices (the black diagonals of decorations), we get a planar (or toric) graph $G_{B}^{T}$. Similarly, removing the white diagonals gives a graph $G_{W}^{T}$. An orientation of $G^{T}$ is said to be admissible if its restriction to $G_{B}^{T}$ and $G_{W}^{T}$ are both Kasteleyn orientation. The existence of such an orientation is established in Section F of [43].

To define a Kasteleyn matrix, we first fix an admissible orientation of $G^{T}$. For any standard $\alpha, \beta: \mathcal{F} \rightarrow \mathbb{R} / 2 \pi \mathbb{Z}$, the $8 \mathrm{~V}$-weights $X_{\alpha, \beta}$ are standard. Thus we can define dimer weights $v_{\alpha, \beta}$ as in Fig. 8 . Let $\tilde{K}_{\alpha, \beta}$ be the weighted, skew-symmetric adjacency matrix associated to the oriented weighted graph $G^{T}$.

In the spherical case, the arguments leading to equation (79) in [43] imply the following. 


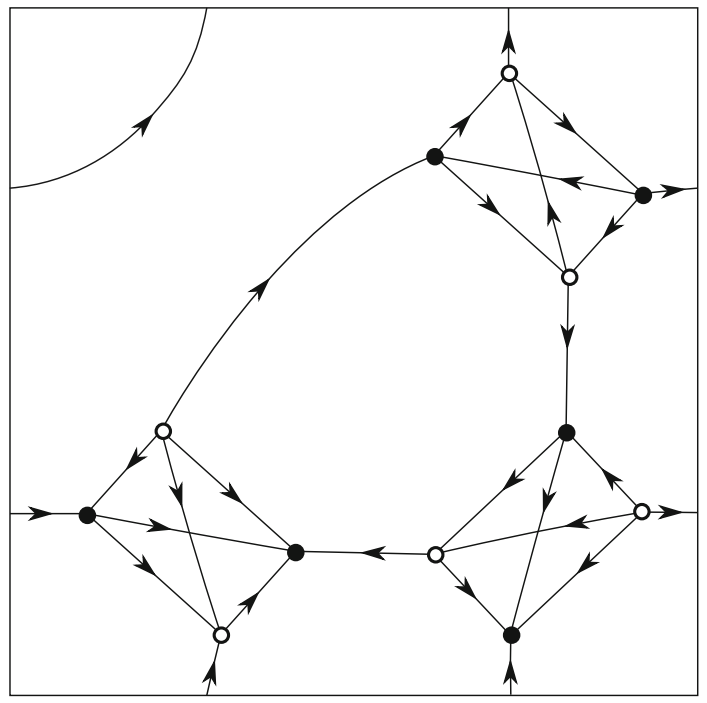

Fig. 9. An admissible orientation of $G^{T}$ in the toric case

Proposition 18 ([42,43]). In the spherical case, for any quadrangulation $\mathcal{Q}$ and any standard $\alpha, \beta: \mathcal{F} \rightarrow \mathbb{R} / 2 \pi \mathbb{Z}$,

$$
\tilde{\mathcal{Z}}_{\operatorname{dim}}\left(G^{T}, v_{\alpha, \beta}\right)=\operatorname{Pf} \tilde{K}_{\alpha, \beta} .
$$

In the toric case, there also exists an admissible orientation, but its Pfaffian is no longer equal to $\tilde{\mathcal{Z}}_{\text {dim }}\left(G^{T}, v_{\alpha, \beta}\right)$. We recall here the standard way of dealing with this problem; the idea was suggested but not proved by Kasteleyn [41,42] and was then proved in various forms of generality in the works of Dolbilin et al. [24], Galluccio and Loebl [36], Tesler [65], Cimasoni and Reshetikhin [20].

Let $m_{0}$ be the dimer configuration consisting of all dimers in the decorations that are parallel to the edges of $G^{\mathcal{B}}$; see the darker configuration of Fig. 12. For any dimer configuration $m$ on $G^{T}$, the superimposition of $m$ and $m_{0}$ is the disjoint union of alternating loops covering all the vertices. This union of curves has a well defined homology in $H_{1}\left(\mathbb{T}^{2}, \mathbb{Z} / 2 \mathbb{Z}\right)$, which we denote $\left(h_{x}^{m}, h_{y}^{m}\right) \in(\mathbb{Z} / 2 \mathbb{Z})^{2}$. For any $\theta, \tau \in\{0,1\}$, let $\tilde{K}_{\alpha, \beta}^{\theta, \tau}$ be the Kasteleyn matrix where the weights of edges of $G^{T}$ crossing $\gamma_{x}^{\mathcal{B}}$ (resp. $\gamma_{y}^{\mathcal{B}}$ ) have been multiplied by $(-1)^{\theta}$ (resp. $\left.(-1)^{\tau}\right)$. Then there exists an admissible orientation such that we have the following.

Proposition 19 ([20,24,36,41,42,65]). In the toric case, for any quadrangulation $\mathcal{Q}$ and any standard $\alpha, \beta: \mathcal{F} \rightarrow \mathbb{R} / 2 \pi \mathbb{Z}$, for any $\theta, \tau \in\{0,1\}$,

$$
\operatorname{Pf} \tilde{K}_{\alpha, \beta}^{\theta, \tau}=\sum_{m \in \mathcal{M}\left(G^{T}\right)}(-1)^{h_{x}^{m} h_{y}^{m}+h_{y}^{m}+h_{y}^{m}+\theta h_{x}^{m}+\tau h_{y}^{m}} \tilde{w}(m) .
$$

Consequently,

$$
\tilde{\mathcal{Z}}_{\text {dim }}\left(G^{T}, v_{\alpha, \beta}\right)=\frac{1}{2}\left(-\operatorname{Pf} \tilde{K}_{\alpha, \beta}^{0,0}+\operatorname{Pf} \tilde{K}_{\alpha, \beta}^{0,1}+\operatorname{Pf} \tilde{K}_{\alpha, \beta}^{1,0}+\operatorname{Pf} \tilde{K}_{\alpha, \beta}^{1,1}\right) .
$$


For any $(z, w) \in\left(\mathbb{C}^{*}\right)^{2}$, consider the modified matrix $\tilde{K}_{\alpha, \beta}(z, w)$, obtained by multiplying the coefficients $\tilde{K}_{\alpha, \beta}[\mathrm{u}, \mathrm{v}]$ by $z\left(\operatorname{resp} z^{-1}\right)$ when the edge uv crosses $\gamma_{x}^{\mathcal{B}}$ from left to right (resp. right to left), and similarly for $w$ and $\gamma_{y}^{\mathcal{B}}$. This leads to the definition of the characteristic polynomial of the eight-vertex as the Laurent polynomial

$$
P_{\alpha, \beta}^{8 V}(z, w)=\operatorname{det} \tilde{K}_{\alpha, \beta}(z, w) .
$$

When $(z, w) \notin\{ \pm 1\}^{2}$, this quantity has no reason to factor as a square product.

We conclude this part with a few remarks on the planar case. Then $\tilde{K}_{\alpha, \beta}$ is an infinite matrix, or equivalently can be seen as an operator on $\mathbb{C}^{V^{T}}$ :

$$
\forall f \in \mathbb{C}^{V^{T}},\left(\tilde{K}_{\alpha, \beta} f\right)[\mathrm{x}]=\sum_{\mathrm{y} \in V^{T}} K_{\alpha, \beta}[\mathrm{x}, \mathrm{y}] f[\mathrm{y}] .
$$

This is well defined because for all $\mathrm{x} \in V^{T}, \tilde{K}_{\alpha, \beta}[\mathrm{x}, \mathrm{y}]$ is zero for all but a finite number of y's.

An inverse of $\tilde{K}_{\alpha, \beta}$ is an infinite matrix $\tilde{K}_{\alpha, \beta}^{-1}$ such that $\tilde{K}_{\alpha, \beta} \tilde{K}_{\alpha, \beta}^{-1}=\mathrm{Id}$ as a matrix product. This is well defined by the previous remark.

When the graph is $\mathbb{Z}^{2}$-periodic, let $G_{1}^{T}=G^{T} / \mathbb{Z}^{2}$ be the fundamental graph. Note that $G_{1}^{T}$ corresponds to the toric case. For any $(z, w) \in \mathbb{C}^{2}$ the subspace $V_{(z, w)}^{T}$ of $(z, w)$-quasiperiodic functions $f$ :

$$
\forall \mathrm{x} \in V_{1}^{T}, \forall(m, n) \in \mathbb{Z}^{2}, f(\mathrm{x}+(m, n))=z^{-m} w^{-n} f(\mathrm{x})
$$

is fixed by $\tilde{K}_{\alpha, \beta}$. The restriction of $\tilde{K}_{\alpha, \beta}$ to this finite-dimensional subspace is equal to the matrix $\tilde{K}_{\alpha, \beta}(z, w)$ defined in the toric case for $G_{1}^{T}$, via the identification of $\mathrm{x} \in V_{1}^{T}$ with the only $(z, w)$-quasiperiodic function $\delta_{\mathrm{X}}(z, w)$ that takes value 1 at $\mathrm{x}$ and 0 at the other vertices $V_{1}^{T}$ of the fundamental domain.

4.3. Skew-hermitian complex matrix. There is another way to define Kasteleyn matrices that is more intrinsic and does not require fixing an orientation, by using instead complex arguments on the edges. Let $K_{\alpha, \beta}$ be the matrix whose entries are indexed by vertices $V^{T}$ and defined by Fig. 10 and by the skew-hermitian condition:

$$
K_{\alpha, \beta}[\mathrm{u}, \mathrm{v}]=-\overline{K_{\alpha, \beta}[\mathrm{v}, \mathrm{u}]} .
$$

The arguments of the entries are inspired by the relation with the Kac-Ward matrix $[18,19,39]$. The "angles" $\left(\phi_{e}\right)_{e \in \mathcal{E}}$ are defined in the following way:

- In the spherical and planar cases, we embed the graph $G^{\mathcal{B}}$ properly in the plane, with straight edges. Then the white vertices of $\mathcal{Q}, \mathcal{W}$, are in bijection with faces of $G^{\mathcal{B}}$, and the edges $\mathcal{E}$ of $\mathcal{Q}$ are in bijection with the corners of $G^{\mathcal{B}}$. For every $e \in \mathcal{E}$, we set $2 \phi_{e}$ to be the direct angle at the corner corresponding to $e$, taken in $[0,2 \pi)$. See Fig. 11.

- In the toric case, we lift $\mathcal{Q}$ to a bipartite periodic quadrangulation of the plane, and we proceed as in the planar case. This yields a periodic choice of angles $\phi$, which can be mapped again to the torus. 


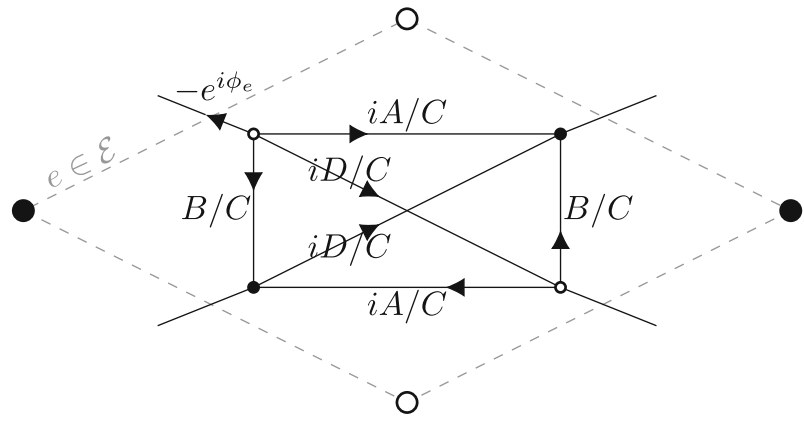

Fig. 10. The skew-hermitian Kasteleyn matrix $K_{\alpha, \beta}$ on $G^{T}$

In the toric case, we also define $K_{\alpha, \beta}(z, w)$ just as before.

The following result relates the matrices $\tilde{K}_{\alpha, \beta}$ and $K_{\alpha, \beta}$ by "gauge equivalence". In particular, it shows that all their principal minors are equal.

Lemma 20. In the spherical and toric cases, there exists a diagonal unitary matrix $\mathcal{D}$, that depends only on the chosen admissible orientation of $G^{T}$, such that

$$
K_{\alpha, \beta}=\mathcal{D}^{-1} \tilde{K}_{\alpha, \beta} \mathcal{D}
$$

Proof. We use Theorem 2.1 of [60]; see also Appendix A of [23]. The existence of such a diagonal (not necessarily unitary) matrix is equivalent to having, for every cycle $\mathcal{C}=\left(\mathrm{x}_{1}, \ldots, \mathrm{x}_{p}, \mathrm{x}_{p+1}=\mathrm{x}_{1}\right)$ of adjacent vertices on $G^{T}$,

$$
\prod_{i=1}^{p} K_{\alpha, \beta}\left[\mathrm{x}_{i}, \mathrm{x}_{i+1}\right]=\prod_{i=1}^{p} \tilde{K}_{\alpha, \beta}\left[\mathrm{x}_{i}, \mathrm{x}_{i+1}\right] .
$$

Since the complex moduli of the entries of $K_{\alpha, \beta}$ and $\tilde{K}_{\alpha, \beta}$ are equal, it is sufficient to show that the complex arguments in (25) are the same. Notice that for the simple cycles $(\mathrm{x}, \mathrm{y}, \mathrm{x})$, by the skew-symmetric and skew-hermitian properties,

$$
\arg \left(K_{\alpha, \beta}[\mathrm{x}, \mathrm{y}] K_{\alpha, \beta}[\mathrm{y}, \mathrm{x}]\right)=\pi=\arg \left(\tilde{K}_{\alpha, \beta}[\mathrm{x}, \mathrm{y}] \tilde{K}_{\alpha, \beta}[\mathrm{y}, \mathrm{x}]\right) .
$$

Moreover, if we show that the right-hand side of (25) is real (i.e. the argument is $0[\pi]$ ), then we only have to check one direction for any cycle.

All in all, by decomposing cycles, it suffices to check that the complex arguments in (25) are equal and real for the following cycles:

1. the cycles that winds once in the counter-clockwise direction around a vertex of $\mathcal{B}$, or of $\mathcal{W}$;

2. the counter-clockwise 3-cycles inside decorations that use two sides and a diagonal;

3 . in the torus case, two fixed cycles that wind once vertically (resp. horizontally) around the torus.

Case 1: let $\mathcal{C}$ be such a cycle corresponding to a black vertex $b \in \mathcal{B}$. Let $\phi_{1}, \ldots, \phi_{p}$ be the successive angles around $b$ as in Fig. 11. By grouping together the successive steps of $\mathcal{C}$ on legs and inside decorations, the argument of the left-hand side of (25) is

$$
\sum_{i=1}^{p} \phi_{i}=\pi
$$



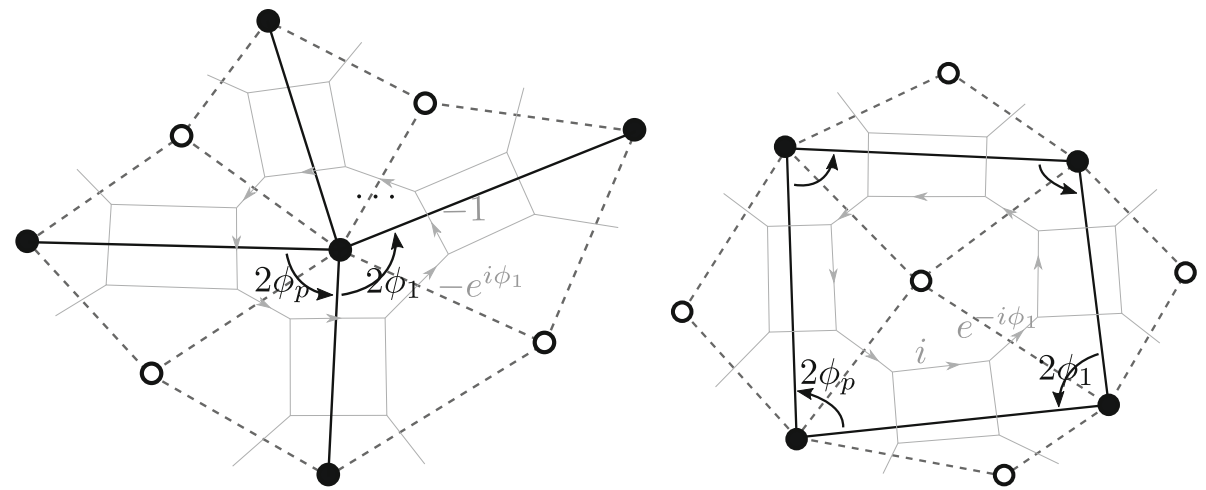

Fig. 11. Embedded graph $G^{\mathcal{B}}$ and unitary part of the entries of $K_{\alpha, \beta}$ on simple cycles around black and white vertices

On the right-hand side, as the cycle is even, the product is also a negative real number for an admissible orientation.

If $\mathcal{C}$ corresponds to a white vertex $w \in \mathcal{W}$, we also set $\phi_{1}, \ldots, \phi_{p}$ to be the successive angles around $w$ as in Fig. 11. Then

$$
\sum_{i=1}^{p} 2 \phi_{i}=(p \pm 2) \pi
$$

with a $-\operatorname{sign}$ when $w$ corresponds to an interior face of $G^{\mathcal{B}}$ when embedded in the plane, and a + sign for the exterior face. Again by grouping the steps, the argument for the product of the left-hand side of (25) is $\sum_{i=1}^{p}\left(\frac{\pi}{2}-\phi_{i}\right)=\pi[2 \pi]$, and we conclude similarly.

Case 2: In Fig. 10 we easily check that for any of these 3-cycles, the argument of the product of the elements of $K_{\alpha, \beta}$ is $\pi[2 \pi]$. By the construction of admissible orientations, these are also clockwise-odd so the product for $\tilde{K}_{\alpha, \beta}$ is also a negative real number.

Case 3: We show this for a cycle that winds once vertically around the torus, the horizontal case being identical. We chose the alternating cycle $\mathcal{C}_{y}$ represented in Fig. 12. This cycle is obtained by superimposing the dimer configuration $m_{0}$ with a configuration $m_{y}$ that uses the legs that cross edges of $\mathcal{Q}$ that touch $\gamma_{y}^{\mathcal{B}}$ on the right, edges parallel to the white diagonal in the decorations of faces containing two such edges of $\mathcal{Q}$, and is equal to $m_{0}$ otherwise.

Again by decomposing the path, one easily checks that the argument on the left-hand side of $(25)$ is

$$
\sum_{i=1}^{p}\left(\psi_{i}-\frac{\pi}{2}\right)=0 .
$$

For the right-hand side, we know that $m_{0}$ has homology $(0,0)$ while $m_{y}$ has homology $(0,1)$, so that by Proposition 19 the term in det $\tilde{K}_{\alpha, \beta}$ corresponding to the superimposition of $m_{0}$ and $m_{y}$ must appear with a minus sign. All the double dimers in this superimposition give a + sign, because the - sign of the product of opposite matrix elements is compensated by the signature of a 2-cycle. Following this reasoning, the 


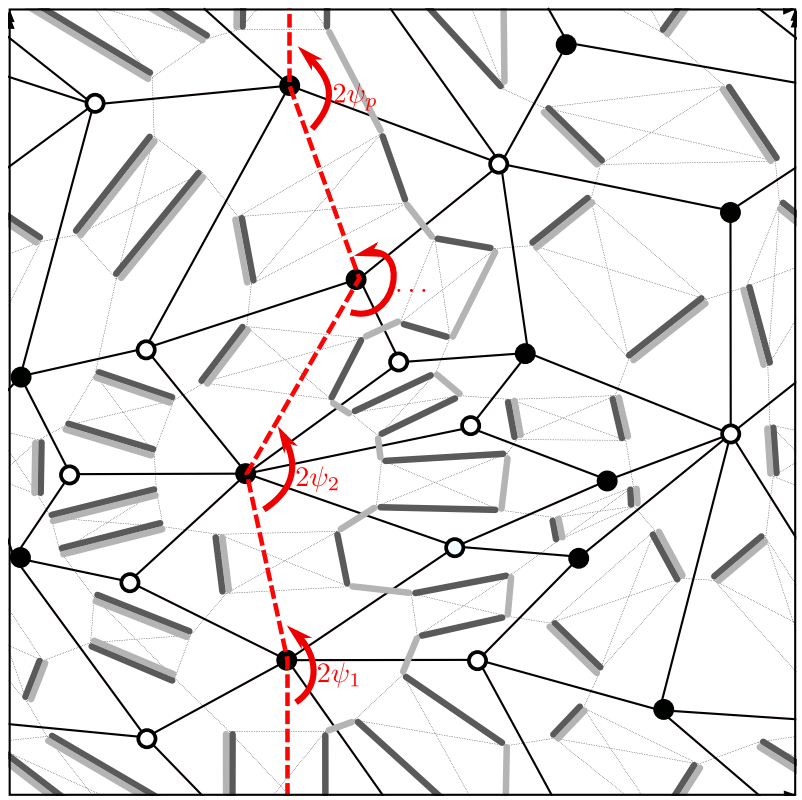

Fig. 12. A quadrangulation of the torus with the path $\gamma_{y}^{\mathcal{B}}$ (dashed); the graph $G^{T}$ equipped with two dimer configurations, $m_{0}$ (dark grey) and $m_{y}$ (light grey)

product corresponding to the alternating cycle $C_{y}$ must be positive, since the signature of the corresponding cycle of the permutation is -1 . This proves that the arguments match.

The fact that $\mathcal{D}$ does not depend on $\alpha, \beta$ is a consequence of the explicit form given in [23]. Finally, to show that the matrix $\mathcal{D}$ can be taken to be unitary, we just have to show that its diagonal elements have the same modulus, since multiplying it by a constant leaves relation (24) unchanged. For any two adjacent vertices $\mathrm{x}, \mathrm{y} \in V^{T}$,

$$
K_{\alpha, \beta}[\mathrm{x}, \mathrm{y}]=\frac{\mathcal{D}[\mathrm{y}, \mathrm{y}]}{\mathcal{D}[\mathrm{x}, \mathrm{x}]} \tilde{K}_{\alpha, \beta}[\mathrm{x}, \mathrm{y}]
$$

so that $|\mathcal{D}[\mathrm{x}, \mathrm{x}]|=|\mathcal{D}[\mathrm{y}, \mathrm{y}]|$. Since the graph is connected, all these moduli are equal.

4.4. Eight-vertex partition function and correlations. By injecting the result of Theorem 17 into Propositions 18 and 19, and using Lemma 20 to transform the determinant of $\tilde{K}_{\alpha, \beta}$ into that of $K_{\alpha, \beta}$ (we cannot do the same for Pfaffian, since the latter is only defined for skew-symmetric matrices $a$ priori) we get

Corollary 21. Let $\mathcal{Q}$ be a quadrangulation and $\alpha, \beta: \mathcal{F} \rightarrow \mathbb{R} / 2 \pi \mathbb{Z}$ be standard. In the spherical case,

$$
\left(\mathcal{Z}_{8 V}\left(\mathcal{Q}, X_{\alpha, \beta}\right)\right)^{2}=\left(\prod_{f \in \mathcal{F}} C_{\alpha, \beta}(f)\right)^{2} \operatorname{det} K_{\alpha, \beta}
$$


In the toric case,

$$
\mathcal{Z}_{8 V}\left(\mathcal{Q}, X_{\alpha, \beta}\right)=\frac{\prod_{f \in \mathcal{F}} C_{\alpha, \beta}(f)}{2}\left(-\operatorname{Pf} \tilde{K}_{\alpha, \beta}^{0,0}+\operatorname{Pf} \tilde{K}_{\alpha, \beta}^{0,1}+\operatorname{Pf} \tilde{K}_{\alpha, \beta}^{1,0}+\operatorname{Pf} \tilde{K}_{\alpha, \beta}^{1,1}\right) .
$$

Another standard result is the computation of dimer statistics in terms of minors of the inverse Kasteleyn matrix; see [44,46]. If we adapt this to the $8 \mathrm{~V}$ statistics, where we are only interested in the statistics of the legs dimers, i.e. dimers that have weight 1 , we obtain:

Proposition 22. ([44]) Let $\mathcal{Q}$ be a quadrangulation in the spherical or toric case. Let $e_{1}, \ldots, e_{p} \in \mathcal{E}$, each $e_{i}$ corresponding to a leg of $G^{T}$, whose endpoints we denote $\mathrm{b}_{i} \in B^{T}$ and $\mathrm{w}_{i} \in W^{T}$. Let $\mathrm{V}=\left\{\mathrm{b}_{1}, \mathrm{w}_{1}, \ldots, \mathrm{b}_{p}, \mathrm{w}_{p}\right\}$.

Let $\alpha, \beta: \mathcal{F} \rightarrow \mathbb{R} / 2 \pi \mathbb{Z}$ satisfy (13). Let $\tau$ be a random $8 V$-configuration with Boltzmann distribution $\mathbb{P}_{8 V}$. Then in the spherical case,

$$
\left(\mathbb{P}_{8 V}\left(\left\{e_{1}, \ldots, e_{p}\right\} \subset \tau\right)\right)^{2}=\operatorname{det}\left[\left(K_{\alpha, \beta}^{-1}\right)_{\mathrm{V}}\right]
$$

where the matrix on the right-hand side is the submatrix of $K_{\alpha, \beta}^{-1}$ with rows and columns indexed by $\mathrm{V}$.

In the toric case,

$$
\begin{aligned}
& \mathbb{P}_{8 V}\left(\left\{e_{1}, \ldots, e_{p}\right\} \subset \tau\right) \\
& \quad=\frac{\prod_{f \in \mathcal{F}} C_{\alpha, \beta}(f)}{2 \mathcal{Z}_{8 V}\left(\mathcal{Q}, X_{\alpha, \beta}\right)}\left(-\operatorname{Pf}\left(\tilde{K}_{\alpha, \beta}^{0,0}\right)_{\mathrm{V}^{c}}+\operatorname{Pf}\left(\tilde{K}_{\alpha, \beta}^{0,1}\right)_{\mathrm{V}^{c}}+\operatorname{Pf}\left(\tilde{K}_{\alpha, \beta}^{1,0}\right)_{\mathrm{V}^{c}}+\operatorname{Pf}\left(\tilde{K}_{\alpha, \beta}^{1,1}\right)_{\mathrm{V}^{c}}\right) .
\end{aligned}
$$

4.5. Relations between matrices $K_{\alpha, \beta}^{-1}$. We now exhibit a symmetry in the $8 \mathrm{~V}$-model in the form of a relation between inverse matrices for different values of $\alpha, \beta$.

4.5.1. Spherical and planar cases Let us define the matrix $T$ with entries indexed by the vertices $V^{T}$ of the dimer graph $G^{T}$, in the following way: if $\mathrm{w} \in W^{T}$, then there is a unique "leg" adjacent to w. Let us denote $\hat{\mathrm{w}} \in B^{T}$ the other endpoint of this leg. Let $e \in \mathcal{E}$ be the edge of $\mathcal{Q}$ crossed by $\{\mathrm{w} \hat{\mathrm{w}}\}$. We define

$$
\begin{aligned}
& T(\mathrm{w}, \hat{\mathrm{w}})=-e^{i \phi_{e}}, \\
& T(\hat{\mathrm{w}}, \mathrm{w})=-e^{-i \phi_{e}},
\end{aligned}
$$

and all the other entries of $T$ are zero. Thus $T$ is a weighted permutation matrix between vertices $\mathrm{x}$ and their associated neighbor, which we still denote $\hat{\mathrm{x}}$, $\mathrm{x}$ being black or white.

Theorem 23. In the spherical case or planar case, let $(\alpha, \beta)$ and $\left(\alpha^{\prime}, \beta^{\prime}\right)$ be standard elements of $\left([0,2 \pi)^{\mathcal{F}}\right)^{2}$. If the matrices $K_{\alpha, \beta^{\prime}}^{-1}, K_{\alpha^{\prime}, \beta}^{-1}$ are inverses of $K_{\alpha, \beta^{\prime}}, K_{\alpha^{\prime}, \beta}$, then the following are inverses of $K_{\alpha, \beta}, K_{\alpha^{\prime}, \beta^{\prime}}$ :

$$
\begin{aligned}
K_{\alpha, \beta}^{-1} & =\frac{1}{2}\left((I+T) K_{\alpha, \beta^{\prime}}^{-1}+(I-T) K_{\alpha^{\prime}, \beta}^{-1}\right), \\
K_{\alpha^{\prime}, \beta^{\prime}}^{-1} & =\frac{1}{2}\left((I-T) K_{\alpha, \beta^{\prime}}^{-1}+(I+T) K_{\alpha^{\prime}, \beta}^{-1}\right) .
\end{aligned}
$$




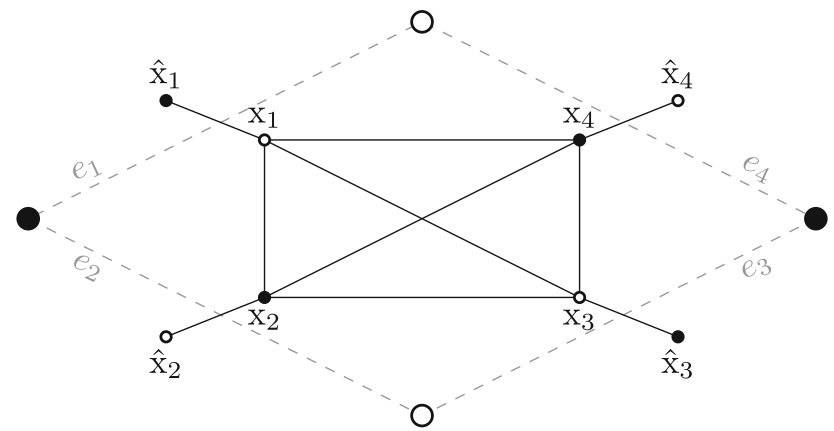

Fig. 13. Notation for $G^{T}$ around $\mathrm{x}_{1} \in W^{T}$

Proof. To simplify notations, we will denote

- $(A, B, C, D)$ the weights $X_{\alpha, \beta}$;

- $\left(A^{\prime}, B^{\prime}, C^{\prime}, D^{\prime}\right)$ the weights $X_{\alpha^{\prime}, \beta^{\prime}}$;

- $(a, b, c, d)$ the weights $X_{\alpha, \beta^{\prime}}$, and $K_{\alpha, \beta^{\prime}}^{-1}=\left(u_{\mathrm{x}, \mathrm{y}}\right)_{\mathrm{x}, \mathrm{y} \in V^{T}}$;

- $\left(a^{\prime}, b^{\prime}, c^{\prime}, d^{\prime}\right)$ the weights $X_{\alpha^{\prime}, \beta}$, and $K_{\alpha^{\prime}, \beta}^{-1}=\left(u_{\mathrm{x}, \mathrm{y}}^{\prime}\right)_{\mathrm{x}, \mathrm{y} \in V^{T}}$.

Lemma 24. The following relations, implicitly evaluated at any $f \in \mathcal{F}$, hold:

$$
\begin{aligned}
& c A+d B-a C-b D=0, c^{\prime} A-d^{\prime} B-a^{\prime} C+b^{\prime} D=0, \\
& d A-c B+b C-a D=0, d^{\prime} A+c^{\prime} B-b^{\prime} C-a^{\prime} D=0 \text {, } \\
& a A+b B-c C-d D=0, a^{\prime} A+b^{\prime} B-c^{\prime} C-d^{\prime} D=0 \text {, } \\
& b A-a B+d C-c D=0, b^{\prime} A-a^{\prime} B-d^{\prime} C+c^{\prime} D=0 \text {. }
\end{aligned}
$$

Proof of Lemma 24. This is done by direct computations, which are made easier by the use of the alternative form of weights (9).

Let $\mathrm{x}_{1} \in W^{T}$. Its neighbors are denoted $\hat{\mathrm{x}}_{1}, \mathrm{x}_{2}, \mathrm{x}_{3}, \mathrm{x}_{4}$ as in Fig. 13. For any $\mathrm{y} \in V^{T}$ and $i \in\{1, \ldots, 4\}$ we have $\left(K_{\alpha, \beta^{\prime}} K_{\alpha, \beta^{\prime}}^{-1}\right)\left[\mathrm{x}_{i}, \mathrm{y}\right]=\delta_{\mathrm{x}_{i}, \mathrm{y}}$, which reads:

$$
\begin{aligned}
-e^{i \phi_{e_{1}}} u_{\hat{\mathrm{x}}_{1}, \mathrm{y}}+\frac{b}{c} u_{\mathrm{x}_{2}, \mathrm{y}}+i \frac{d}{c} u_{\mathrm{x}_{3}, \mathrm{y}}+i \frac{a}{c} u_{\mathrm{x}_{4}, \mathrm{y}} & =\delta_{\mathrm{x}_{1}, \mathrm{y}} \\
-\frac{b}{c} u_{\mathrm{x}_{1}, \mathrm{y}}+e^{-i \phi_{e_{2}}} u_{\mathrm{x}_{2}, \mathrm{y}}+i \frac{a}{c} u_{\mathrm{x}_{3}, \mathrm{y}}+i \frac{d}{c} u_{\mathrm{x}_{4}, \mathrm{y}} & =\delta_{\mathrm{x}_{2}, \mathrm{y}} \\
i \frac{d}{c} u_{\mathrm{x}_{1}, \mathrm{y}}+i \frac{a}{c} u_{\mathrm{x}_{2}, \mathrm{y}}-e^{i \phi_{e_{3}}} u_{\hat{\mathrm{x}}_{3}, \mathrm{y}}+\frac{b}{c} u_{\mathrm{x}_{4}, \mathrm{y}} & =\delta_{\mathrm{x}_{3}, \mathrm{y}} \\
i \frac{a}{c} u_{\mathrm{x}_{1}, \mathrm{y}}+i \frac{d}{c} u_{\mathrm{x}_{2}, \mathrm{y}}-\frac{b}{c} u_{\mathrm{x}_{3}, \mathrm{y}}+e^{-i \phi_{e_{4}}} u_{\hat{\mathrm{x}}_{4}, \mathrm{y}} & =\delta_{\mathrm{x}_{4}, \mathrm{y}}
\end{aligned}
$$

By writing the same equations for $K_{\alpha^{\prime}, \beta}$, we get the same relations where $u_{\mathrm{x}, \mathrm{y}}$ is changed into $u_{\mathrm{x}, \mathrm{y}}^{\prime}$ and $(a, b, c, d)$ are changed into $\left(a^{\prime}, b^{\prime}, c^{\prime}, d^{\prime}\right)$. We denote these four new equations by $\left(E_{1}^{\prime}\right),\left(E_{2}^{\prime}\right),\left(E_{3}^{\prime}\right),\left(E_{4}^{\prime}\right)$.

Now we compute

$$
\begin{aligned}
& C\left(E_{1}\right)-B\left(E_{2}\right)+i D\left(E_{3}\right)-i A\left(E_{4}\right) \\
& \quad+C\left(E_{1}^{\prime}\right)+B\left(E_{2}^{\prime}\right)-i D\left(E_{3}^{\prime}\right)+i A\left(E_{4}^{\prime}\right) .
\end{aligned}
$$


On the right-hand side, this is $2 C \delta_{\mathrm{x}_{1}, \mathrm{y}}$. On the left-hand side, we can group the terms corresponding to the same $u_{\mathrm{x}, \mathrm{y}}\left(\right.$ or $\left.u_{\mathrm{x}, \mathrm{y}}^{\prime}\right)$. For instance, $u_{\mathrm{x}_{1}, \mathrm{y}}$ will appear with coefficient

$$
\frac{b}{c} B-\frac{d}{c} D+\frac{a}{c} A
$$

which is equal to $C$ according to Lemma 24. All in all, using all relations of Lemma 24, this yields

$$
\begin{aligned}
& -C e^{i \phi_{e_{1}}}\left(u_{\hat{\mathrm{x}}_{1}, \mathrm{y}}+u_{\hat{\mathrm{x}}_{1}, \mathrm{y}}^{\prime}-e^{-i \phi_{e_{1}}}\left(u_{\mathrm{x}_{1}, y}-u_{\mathrm{x}_{1}, y}^{\prime}\right)\right) \\
& +B\left(u_{\mathrm{x}_{2}, \mathrm{y}}+u_{\mathrm{x}_{2}, \mathrm{y}}^{\prime}-e^{-i \phi_{e_{2}}}\left(u_{\hat{\mathrm{x}}_{2}, y}-u_{\hat{\mathrm{x}}_{2}, y}^{\prime}\right)\right) \\
& +i D\left(u_{\mathrm{x}_{3}, \mathrm{y}}+u_{\mathrm{x}_{3}, \mathrm{y}}^{\prime}-e^{i \phi_{e_{3}}}\left(u_{\hat{\mathrm{x}}_{3}, y}-u_{\hat{\mathrm{x}}_{3}, y}^{\prime}\right)\right) \\
& +i A\left(u_{\mathrm{x}_{4}, \mathrm{y}}+u_{\mathrm{x}_{4}, \mathrm{y}}^{\prime}-e^{-i \phi_{e_{4}}}\left(u_{\hat{\mathrm{x}}_{4}, y}-u_{\hat{\mathrm{x}}_{4}, y}^{\prime}\right)\right)=2 C \delta_{\mathrm{x} 1, \mathrm{y}} .
\end{aligned}
$$

For $\mathrm{x}, \mathrm{y} \in V^{T}$, let $e_{\mathrm{x}} \in \mathcal{E}$ be the edge of the quadrangulation closest to x, and let $M_{\mathrm{x}, \mathrm{y}}$ be

$$
\begin{aligned}
& \text { if } \mathrm{x} \in W^{T}, \quad M_{\mathrm{x}, \mathrm{y}}=\frac{1}{2}\left(u_{\mathrm{x}, \mathrm{y}}+u_{\mathrm{x}, \mathrm{y}}^{\prime}-e^{i \phi_{e_{\mathrm{x}}}}\left(u_{\hat{\mathrm{x}}, \mathrm{y}}-u_{\hat{\mathrm{x}}, \mathrm{y}}^{\prime}\right)\right), \\
& \text { if } \mathrm{x} \in B^{T}, \quad M_{\mathrm{x}, \mathrm{y}}=\frac{1}{2}\left(u_{\mathrm{x}, \mathrm{y}}+u_{\mathrm{x}, \mathrm{y}}^{\prime}-e^{-i \phi_{e_{\mathrm{x}}}}\left(u_{\hat{\mathrm{x}}, \mathrm{y}}-u_{\hat{\mathrm{x}}, \mathrm{y}}^{\prime}\right)\right) .
\end{aligned}
$$

Then Eq. (36) exactly means that the matrix $M=\left(M_{\mathrm{x}, \mathrm{y}}\right)_{\mathrm{x}, \mathrm{y} \in V^{T}}$ satisfies

$$
\left(K_{\alpha, \beta} M\right)\left[\mathrm{x}_{1}, \mathrm{y}\right]=\delta_{\mathrm{x}_{1}, \mathrm{y}}
$$

when $\mathrm{x}_{1} \in W^{T}$. A similar computation shows that (38) also holds when $\mathrm{x}_{1} \in B^{T}$. As a result, $M$ is an inverse of $K_{\alpha, \beta}$, and (37) is equivalent to

$$
M=\frac{1}{2}\left[(I+T) K_{\alpha, \beta^{\prime}}^{-1}+(I-T) K_{\alpha^{\prime}, \beta}^{-1}\right] .
$$

The second matrix relation in (31) is obtained by switching $(\alpha, \beta) \leftrightarrow\left(\alpha^{\prime}, \beta^{\prime}\right)$.

Remark 25. Theorem 23 can be used to give an alternative proof of the relation of partition functions (22). This works exactly as in the forthcoming proof of the analogous statement for toric quadrangulations, see Theorem 27.

\subsubsection{Toric case}

Theorem 26. Let $\mathcal{Q}$ be a quadrangulation in the toric case. Let $(\alpha, \beta)$ and $\left(\alpha^{\prime}, \beta^{\prime}\right)$ be two standard elements of $\left([0,2 \pi)^{\mathcal{F}}\right)^{2}$.

Let $(z, w) \in\left(\mathbb{C}^{*}\right)^{2}$ be such that $K_{\alpha, \beta^{\prime}}(z, w)$ and $K_{\alpha^{\prime}, \beta}(z, w)$ are invertible. Then $K_{\alpha, \beta}(z, w)$ and $K_{\alpha^{\prime}, \beta^{\prime}}(z, w)$ are invertible and their inverses are given by

$$
\begin{aligned}
K_{\alpha, \beta}^{-1}(z, w) & =\frac{1}{2}\left[(I+T) K_{\alpha, \beta^{\prime}}^{-1}(z, w)+(I-T) K_{\alpha^{\prime}, \beta}^{-1}(z, w)\right], \\
K_{\alpha^{\prime}, \beta^{\prime}}^{-1}(z, w) & =\frac{1}{2}\left[(I-T) K_{\alpha, \beta^{\prime}}^{-1}(z, w)+(I+T) K_{\alpha^{\prime}, \beta}^{-1}(z, w)\right] .
\end{aligned}
$$


Proof. The proof, being based on a local computation of matrix products, is identical to that of Theorem 23. One simply has to take into account the possible multiplication by $z^{ \pm 1}$ and $w^{ \pm 1}$ in the weights when the face considered is crossed by $\gamma_{x}^{\mathcal{B}}, \gamma_{y}^{\mathcal{W}}$, or both. For instance, if it is crossed by $\gamma_{x}^{\mathcal{B}}$, in the notation of the proof of Theorem 23 , one has to compute

$$
\begin{aligned}
& C\left(E_{1}\right)-z B\left(E_{2}\right)+i z D\left(E_{3}\right)-i A\left(E_{4}\right) \\
& \quad+C\left(E_{1}^{\prime}\right)+z B\left(E_{2}^{\prime}\right)-i z D\left(E_{3}^{\prime}\right)+i A\left(E_{4}^{\prime}\right) .
\end{aligned}
$$

to get the correct matrix relation. The other cases are similar.

Theorem 27. Let $\mathcal{Q}$ be a quadrangulation in the toric case. Let $(\alpha, \beta)$ and $\left(\alpha^{\prime}, \beta^{\prime}\right)$ be two standard elements of $\left([0,2 \pi)^{\mathcal{F}}\right)^{2}$. Then the characteristic polynomials satisfy

$$
P_{\alpha, \beta}^{8 V} P_{\alpha^{\prime}, \beta^{\prime}}^{8 V}=c_{2} P_{\alpha, \beta^{\prime}}^{8 V} P_{\alpha^{\prime}, \beta}^{8 V}
$$

where

$$
c_{2}=\frac{c_{\alpha, \beta^{\prime}} c_{\alpha^{\prime}, \beta}}{c_{\alpha, \beta} c_{\alpha^{\prime}, \beta^{\prime}}}=\prod_{f \in \mathcal{F}} \frac{C_{\alpha, \beta^{\prime}}(f) C_{\alpha^{\prime}, \beta}(f)}{C_{\alpha, \beta}(f) C_{\alpha^{\prime}, \beta^{\prime}}(f)} .
$$

To prove Theorem 27, we also need the following diagonal matrix $D$, whose rows and columns are indexed by the vertices of $G^{T}$ :

$$
D_{\mathrm{xx}}= \begin{cases}-1 & \text { if } \mathrm{x} \in W^{T} \\ 1 & \text { if } \mathrm{x} \in B^{T}\end{cases}
$$

Lemma 28. Let $\mathcal{Q}$ be a quadrangulation in the toric case, and let $\alpha, \beta: \mathcal{F} \rightarrow[0,2 \pi[$ be standard, and $(z, w) \in\left(\mathbb{C}^{*}\right)^{2}$. The commutator of $K_{\alpha, \beta}(z, w)$ with $T$ is

$$
\left[K_{\alpha, \beta}(z, w), T\right]=-K_{\alpha, \beta}(z, w) D K_{\alpha, \beta}(z, w) .
$$

If $P_{\alpha, \beta}(z, w) \neq 0$, the commutator of $K_{\alpha, \beta}^{-1}(z, w)$ with $T$ is

$$
\left[K_{\alpha, \beta}^{-1}(z, w), T\right]=D
$$

Proof. Equality (39) can be verified by a straightforward computation of the matrix elements. For instance, in the notations of Fig. 13 (we drop the $(z, w)$ in the computation to simplify notations) the matrix element $\left[\mathrm{x}_{1}, \hat{\mathrm{x}}_{2}\right]$ are

$$
\begin{aligned}
\left(K_{\alpha, \beta} T\right)\left[\mathrm{x}_{1}, \hat{\mathrm{x}}_{2}\right]-\left(T K_{\alpha, \beta}\right)\left[\mathrm{x}_{1}, \hat{\mathrm{x}}_{2}\right] & =K_{\alpha, \beta}\left[\mathrm{x}_{1}, \mathrm{x}_{2}\right] T\left[\mathrm{x}_{2}, \hat{\mathrm{x}}_{2}\right]-0 \\
& =\frac{B}{C}\left(-e^{-i \phi_{e_{2}}}\right) ; \\
-\left(K_{\alpha, \beta} D K_{\alpha, \beta}\right)\left[\mathrm{x}_{1}, \hat{\mathrm{x}}_{2}\right] & =-K_{\alpha, \beta}\left[\mathrm{x}_{1}, \mathrm{x}_{2}\right] D\left[\mathrm{x}_{2}, \mathrm{x}_{2}\right] K_{\alpha, \beta}\left[\mathrm{x}_{2}, \hat{\mathrm{x}}_{2}\right] \\
& =-\frac{B}{C}\left(e^{-i \phi_{e_{2}}}\right) .
\end{aligned}
$$

Another important case is the matrix element $\left[\mathrm{x}_{1}, \mathrm{x}_{1}\right]$ :

$$
\left(K_{\alpha, \beta} T\right)\left[\mathrm{x}_{1}, \mathrm{x}_{1}\right]-\left(T K_{\alpha, \beta}\right)\left[\mathrm{x}_{1}, \mathrm{x}_{1}\right]
$$




$$
\begin{aligned}
& =K_{\alpha, \beta}\left[\mathrm{x}_{1}, \hat{\mathrm{x}_{1}}\right] T\left[\hat{\mathrm{x}}_{1}, \mathrm{x}_{1}\right]-T\left[\mathrm{x}_{1}, \hat{\mathrm{x}_{1}}\right] K_{\alpha, \beta}\left[\hat{\mathrm{x}_{1}}, \mathrm{x}_{1}\right] \\
& =\left(-e^{i \phi_{e_{1}}}\right)\left(-e^{-i \phi_{e_{1}}}\right)-\left(-e^{i \phi_{e_{1}}}\right) e^{-i \phi_{e_{1}}} ; \\
& =2 \\
- & \left(K_{\alpha, \beta} D K_{\alpha, \beta}\right)\left[\mathrm{x}_{1}, \hat{\mathrm{x}}_{2}\right] \\
& =-\left(\left(-e^{i \phi_{e_{1}}}\right) e^{-i \phi_{e_{1}}}+\frac{B}{C}\left(-\frac{B}{C}\right)+i \frac{D}{C}(-1) i \frac{D}{C}+i \frac{A}{C} i \frac{A}{C}\right) \\
& =-\left(-1+\frac{-B^{2}-A^{2}+D^{2}}{C^{2}}\right) \\
& =2 .
\end{aligned}
$$

All other cases are similar.

For the second point, $P(z, w)=\operatorname{det}\left(\tilde{K}_{\alpha, \beta}(z, w)\right)=\operatorname{det}\left(K_{\alpha, \beta}(z, w)\right)$ Lemma 20, so the matrix $K_{\alpha, \beta}(z, w)$ is invertible. Then (40) is simply obtained by multiplying (39) by $K_{\alpha, \beta}^{-1}(z, w)$ on both sides.

Proof of Theorem 27. We prove the polynomials relation for $(z, w)$ such that none of the four polynomials is zero at $(z, w)$ (i.e. the four Kasteleyn matrices are invertible); the relation is then obtained by analytic continuation. By noting that $T^{2}=I$, we can rewrite Theorem 26 as a block-matrix relation:

$$
\left(\begin{array}{cc}
I & I \\
I & -I
\end{array}\right)\left(\begin{array}{cc}
K_{\alpha, \beta}^{-1}(z, w) & 0 \\
K_{\alpha^{\prime}, \beta^{\prime}}^{-1}(z, w) & K_{\alpha^{\prime}, \beta^{\prime}}^{-1}(z, w)
\end{array}\right)=\left(\begin{array}{cc}
I & I \\
T & -T
\end{array}\right)\left(\begin{array}{cc}
K_{\alpha, \beta^{\prime}}^{-1}(z, w) & \frac{1}{2}(I-T) K_{\alpha, \beta^{\prime}}^{-1}(z, w) \\
K_{\alpha^{\prime}, \beta}^{-1}(z, w) & \frac{1}{2}(I+T) K_{\alpha^{\prime}, \beta}^{-1}(z, w)
\end{array}\right)
$$

We take the determinant of these. The matrices $\left(\begin{array}{cc}I & I \\ I & -I\end{array}\right)$ and $\left(\begin{array}{cc}I & I \\ T & -T\end{array}\right)$ can be written in block-diagonal form, with blocks corresponding to the two copies of the pair $(\mathrm{x}, \hat{\mathrm{x}})$ for $\mathrm{x} \in W^{T}$. For the two matrices, the blocks have determinant 4 and there are $2 \mathcal{F}$ blocks, so both their determinants are equal to $2^{4 \mathcal{F}}$.

The determinant of both sides of (41) can now be computed; we successively use the formula for determinants of block matrices, Lemma 28 to exchange $T$ and the matrices $K_{., .}^{-1}(z, w)$, and $\operatorname{det}(D)=(-1)^{2 \mathcal{F}}=1$; we drop the $(z, w)$ in the notations to make the computation clearer:

$$
\begin{aligned}
\left|K_{\alpha, \beta}^{-1}\right|\left|K_{\alpha^{\prime}, \beta^{\prime}}^{-1}\right| & =\left|K_{\alpha^{\prime}, \beta}^{-1}\right|\left|\frac{1}{2}(I-T) K_{\alpha, \beta^{\prime}}^{-1}-K_{\alpha, \beta^{\prime}}^{-1} K_{\alpha^{\prime}, \beta} \frac{1}{2}(I+T) K_{\alpha^{\prime}, \beta}^{-1}\right| \\
& =\left|K_{\alpha^{\prime}, \beta}^{-1}\right|\left|K_{\alpha, \beta^{\prime}}^{-1}\right|\left|-\frac{1}{2}\left(K_{\alpha, \beta^{\prime}} T K_{\alpha, \beta^{\prime}}^{-1}+K_{\alpha^{\prime}, \beta} T K_{\alpha^{\prime}, \beta}^{-1}\right)\right| \\
& =\left|K_{\alpha^{\prime}, \beta}^{-1}\right|\left|K_{\alpha, \beta^{\prime}}^{-1}\right|\left|-T+\frac{1}{2}\left(K_{\alpha, \beta^{\prime}}+K_{\alpha^{\prime}, \beta}\right) D\right| \\
& =\left|K_{\alpha^{\prime}, \beta}^{-1}\right|\left|K_{\alpha, \beta^{\prime}}^{-1}\right|\left|-T D+\frac{1}{2}\left(K_{\alpha, \beta^{\prime}}+K_{\alpha^{\prime}, \beta}\right)\right| .
\end{aligned}
$$




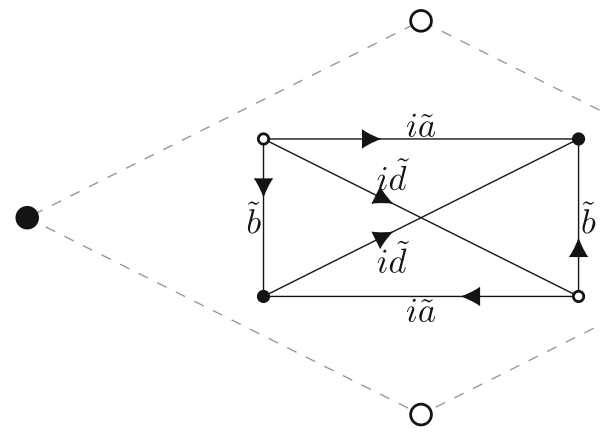

Fig. 14. Coefficients of the matrix $-T D+\frac{1}{2}\left(K_{\alpha, \beta^{\prime}}+K_{\alpha^{\prime}, \beta}\right)$ at a face

Notice that $T D$ is exactly equal the part of $K_{\alpha, \beta}$ that corresponds to legs of $G^{T}$. Thus $-T D+\frac{1}{2}\left(K_{\alpha, \beta^{\prime}}+K_{\alpha^{\prime}, \beta}\right)$ is a block-diagonal matrix, where blocks correspond to decorations inside of the faces $\mathcal{F}$ of $\mathcal{Q}$. When the face is not crossed by $\gamma_{x}^{\mathcal{B}}$ nor $\gamma_{y}^{\mathcal{B}}$, the block we get is represented in Fig. 14, where (in the notation of the proof of Theorem 23):

$$
\begin{aligned}
& \tilde{a}=\frac{1}{2}\left(\frac{a}{c}+\frac{a^{\prime}}{c^{\prime}}\right) \\
& \tilde{b}=\frac{1}{2}\left(\frac{b}{c}+\frac{b^{\prime}}{c^{\prime}}\right) \\
& \tilde{d}=\frac{1}{2}\left(\frac{d}{c}+\frac{d^{\prime}}{c^{\prime}}\right)
\end{aligned}
$$

The determinant of this block can be easily computed using (9), giving

$$
\begin{aligned}
\left(\tilde{a}^{2}+\tilde{b}^{2}-\tilde{d}^{2}\right)^{2} & =\frac{1}{4}\left(1+\frac{a}{c} \frac{a^{\prime}}{c^{\prime}}+\frac{b}{c} \frac{b^{\prime}}{c^{\prime}}-\frac{d}{c} \frac{d^{\prime}}{c^{\prime}}\right)^{2} \\
& =\frac{\frac{a^{2}+b^{2}}{c^{2}} \frac{a^{\prime 2}+b^{\prime 2}}{c^{\prime 2}}}{\frac{A^{2}+B^{2}}{C^{2}} \frac{A^{\prime 2}+B^{\prime 2}}{C^{\prime 2}}}
\end{aligned}
$$

When the face is crossed by $\gamma_{x}^{\mathcal{B}}$ or $\gamma_{y}^{\mathcal{B}}$, some weights are multiplied by $z^{ \pm 1}, w^{ \pm 1}$ but the determinant is the same.

All in all, (42) becomes

$$
\begin{aligned}
& \left(\prod_{f \in \mathcal{F}} \frac{A(f)^{2}+B(f)^{2}}{C(f)^{2}} \frac{A^{\prime}(f)^{2}+B^{\prime}(f)^{2}}{C^{\prime}(f)^{2}}\right)\left|K_{\alpha, \beta}^{-1}(z, w)\right|\left|K_{\alpha^{\prime}, \beta^{\prime}}^{-1}(z, w)\right| \\
& =\left(\prod_{f \in \mathcal{F}} \frac{a(f)^{2}+b(f)^{2}}{c(f)^{2}} \frac{a^{\prime}(f)^{2}+b^{\prime}(f)^{2}}{c^{\prime}(f)^{2}}\right)\left|K_{\alpha, \beta^{\prime}}^{-1}(z, w)\right|\left|K_{\alpha^{\prime}, \beta}^{-1}(z, w)\right| .
\end{aligned}
$$

Using relation (11) finishes the proof. 
An important case appears when we set $\alpha=\beta^{\prime}$ and $\beta=\alpha^{\prime}$. The model with weights $X_{\alpha, \alpha}$ is actually a $6 \mathrm{~V}$ model, and the weights of diagonals in $G^{T}$ become null. This gives the bipartite decorated graph of Wu and Lin [69], see also [15,26] which we denote $G^{Q}$.

More precisely, our Kasteleyn skew-hermitian matrix $K_{\alpha, \alpha}(z, w)$ can be related to Boutillier, de Tilière and Raschel's $\mathcal{K}$ matrix from Section 5 of [16] - whose rows are indexed by white vertices and columns by black vertices of $G^{Q}-$ via

$$
K_{\alpha, \alpha}(z, w)=i\left(\begin{array}{cc}
W^{T} & B^{T} \\
0 & \overline{\mathcal{K}}_{\alpha}(z, w) \\
{ }^{t} \mathcal{K}_{\alpha}\left(z^{-1}, w^{-1}\right) & 0
\end{array}\right){ }_{B^{T}}^{W^{T}} .
$$

The determinant of $\mathcal{K}(z, w)$ is the characteristic polynomial of a bipartite dimer model; we denote it by $P_{\alpha}^{6 V}(z, w)$. Thus $P_{\alpha}^{6 V}(z, w)$ is the determinant of a matrix twice as small as $K_{\alpha, \beta}(z, w)$.

Corollary 29. Let $\mathcal{Q}$ be a quadrangulation in the toric case. Let $(\alpha, \beta)$ and $\left(\alpha^{\prime}, \beta^{\prime}\right)$ be two standard elements of $\left([0,2 \pi)^{\mathcal{F}}\right)^{2}$. Then the characteristic polynomial of the $8 V$-model satisfies

$$
P_{\alpha, \beta}^{8 V}=\tilde{c} P_{\alpha}^{6 V} P_{\beta}^{6 V}
$$

for some constant $\tilde{c}$ satisfying

$$
|\tilde{c}|=\prod_{f \in \mathcal{F}} \frac{2}{\left|C_{\alpha, \beta}(f)\right|} .
$$

Proof. Equation (43) yields

$$
P_{\alpha, \alpha}^{8 V}(z, w)=\overline{P_{\alpha}^{6 V}}(z, w) P_{\alpha}^{6 V}\left(z^{-1}, w^{-1}\right) .
$$

However, $P_{\alpha}^{6 V}$ has extra symmetries. First, as it is the characteristic polynomial of a (bipartite) dimer model, up to a global factor its entries are real (see for instance Proposition 3.1 in [48]), so that $\overline{P_{\alpha}^{6 V}}=c_{3} P_{\alpha}^{6 V}$ for some constant $c_{3} \in S^{1}$. It also corresponds to the dimer model on the decorated graph $G^{Q}$, and the characteristic polynomial in that case is proportional to that of Fisher's decorated graph [34] (see Sect. 4 of [26]). By Corollary 16 of [16], the characteristic polynomial on Fisher's graph has a symmetry $(z, w) \leftrightarrow\left(z^{-1}, w^{-1}\right)$. This gives $P_{\alpha}^{6 V}(z, w)=P_{\alpha}^{6 V}\left(z^{-1}, w^{-1}\right)$. As a result (44) becomes

$$
P_{\alpha, \alpha}^{8 V}=c_{3}\left(P_{\alpha}^{6 V}\right)^{2}
$$

We can now apply Theorem 27 with $\alpha=\beta^{\prime}$ and $\beta=\alpha^{\prime}$. By the same argument as for (14), $P_{\alpha, \beta}^{8 V}=P_{\beta, \alpha}^{8 V}$. Thus Theorem 27 becomes

$$
\left(P_{\alpha, \beta}^{8 V}\right)^{2}=c_{2} c_{3}^{2}\left(P_{\alpha}^{6 V} P_{\beta}^{6 V}\right)^{2}
$$

By analytic continuation and by computing the constant we get the desired relation. 


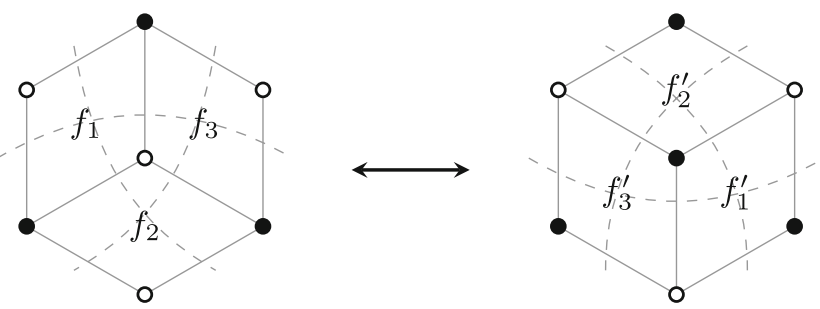

Fig. 15. "Star-triangle" move on the quadrangulation (solid lines) and its dual on which the 8V-configurations are defined (dashed lines)

\section{Z-Invariant Regime}

In this section we restrict to the planar case. The graph may be periodic (in which case we will still make use of the toric case) or not. We study the $Z$-invariant regime of the model, which is a regime where the star-triangle relations are satisfied.

5.1. Checkerboard Yang-Baxter equations. Here we generalize Baxter's star-triangle relations $[5,6]$ in our "checkerboard" setting, and we find free-fermion solutions.

Let us suppose that the quadrangulation $\mathcal{Q}$ contains three adjacent faces in the configuration on the left of Fig. 15. Then we can transform it locally into the configuration on the right. We need to update the weights of the eight-vertex model at the same time. This can be done in such a way that there exists a coupling of the configurations on the right and of the left quadrangulations, such that they agree everywhere except at the central dashed "triangles".

Specifically, let us denote $\left(a_{i}, b_{i}, c_{i}, d_{i}\right)$ the $8 \mathrm{~V}$-weights at $f_{i}$, and $\left(a_{i}^{\prime}, b_{i}^{\prime}, c_{i}^{\prime}, d_{i}^{\prime}\right)$ those at $f_{i}^{\prime}$. By conditioning on every possible boundary condition, we get the following equations for the existence of a coupling: for every $i, j, k$ with $\{i, j, k\}=\{1,2,3\}$,

$$
\begin{aligned}
& c_{i} c_{j} c_{k}+a_{i} a_{j} a_{k} \propto c_{i}^{\prime} c_{j}^{\prime} c_{k}^{\prime}+b_{i}^{\prime} b_{j}^{\prime} b_{k}^{\prime} \\
& a_{i} c_{j} c_{k}+c_{i} a_{j} a_{k} \propto c_{i}^{\prime} a_{j}^{\prime} a_{k}^{\prime}+b_{i}^{\prime} d_{j}^{\prime} d_{k}^{\prime} \\
& c_{i} b_{j} b_{k}+a_{i} d_{j} d_{k} \propto b_{i}^{\prime} c_{j}^{\prime} c_{k}^{\prime}+c_{i}^{\prime} b_{j}^{\prime} b_{k}^{\prime} \\
& c_{i} d_{j} d_{k}+a_{i} b_{j} b_{k} \propto c_{i}^{\prime} d_{j}^{\prime} d_{k}^{\prime}+b_{i}^{\prime} a_{j}^{\prime} a_{k}^{\prime} \\
& c_{i} b_{j} d_{k}+a_{i} d_{j} b_{k} \propto d_{i}^{\prime} a_{j}^{\prime} c_{k}^{\prime}+a_{i}^{\prime} d_{j}^{\prime} b_{k}^{\prime}
\end{aligned}
$$

where the proportionality constants are all the same. We call equations (45) the YangBaxter equations of our model.

Remark 30.

- Most of the equations (all but the last one) are invariant under some nontrivial subgroup of the permutation of indices $\{i, j, k\}$. All in all (45) contains 16 distinct equations.

- We presented the "star-triangle" move as going from the left configuration to the right one, but it can of course be done in both ways, giving the same set of equations.

Equations (45) are often written in matrix form. For the checkerboard setting, we define $R$ and $\bar{R}$ matrices containing the weights at every face, with the indexing of Fig. 16: 

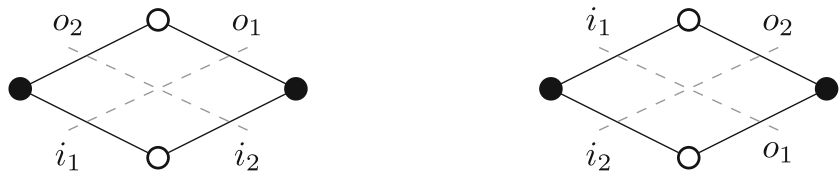

Fig. 16. Entries of $R(f)$ (left) and $\bar{R}(f)$ (right) are indexed by the occupation state of $\left(i_{1}, i_{2}\right)$ and $\left(o_{1}, o_{2}\right)$, in the order (absent, absent), (absent, present), (present, absent), (present, present)

$$
\begin{aligned}
& R(f)=\underset{\sim}{\sim}\left(\begin{array}{cccc}
C(f) & 0 & 0 & A(f) \\
0 & D(f) & B(f) & 0 \\
0 & B(f) & D(f) & 0 \\
A(f) & 0 & 0 & C(f)
\end{array}\right) \text {, }
\end{aligned}
$$

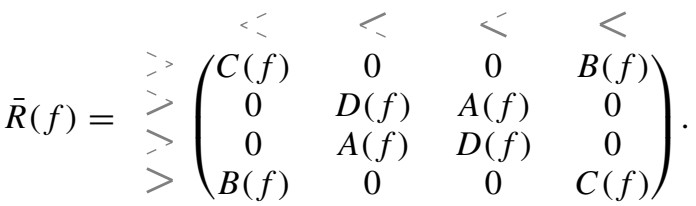

These matrices are elements of $\operatorname{End}(V \otimes V)$, where $V$ is a complex vector space of dimension 2. For $i, j \in\{1,2,3\}, i<j$, we define $\mathcal{R}_{i, j}(f) \in \operatorname{End}(V \otimes V \otimes V)$ that acts as $R(f)$ on the components $i$ and $j$, and as the identity on the other component. We similarly define $\overline{\mathcal{R}}_{i, j}(f)$. Then equations (45) are equivalent to (see for instance [59])

$$
\mathcal{R}_{1,2}\left(f_{1}\right) \overline{\mathcal{R}}_{1,3}\left(f_{2}\right) \mathcal{R}_{2,3}\left(f_{3}\right) \propto \overline{\mathcal{R}}_{2,3}\left(f_{3}^{\prime}\right) \mathcal{R}_{1,3}\left(f_{2}^{\prime}\right) \overline{\mathcal{R}}_{1,2}\left(f_{1}^{\prime}\right) .
$$

5.2. Lozenge graphs. One way to make sure that (45) always hold is to make the $8 \mathrm{~V}$ weights depend on the geometry of the embedding. This has been done for several models on special embedded graphs called isoradial; see for instance [45]. In our context it is more natural to talk only about lozenge graphs.

We say that the planar quadrangulation $\mathcal{Q}$ is a lozenge graph if it is embedded in such a way that all faces are nondegenerate rhombi, with edge length equal to 1 . Then for every $f \in \mathcal{F}$, there is a natural parameter $\theta(f) \in(0, \pi / 2)$, which is the half-angle of the black corners of the rhombus. For a vertex $\mathrm{x} \in V^{T}$, we also denote $\theta(\mathrm{x})=\theta(f)$ where $f$ is the face containing $\mathrm{x}$.

A lozenge graph $\mathcal{Q}$ is said to be quasicrystalline if the number $l$ of possible directions $\pm e^{i \alpha}$ of the edges of the rhombi is finite. In that case there exists an $\epsilon>0$ such that for all faces $f, \theta(f) \in\left(\epsilon, \frac{\pi}{2}-\epsilon\right)$.

Let $k$ be a complex number such that $k^{2} \in(-\infty, 1)$, which will serve as an elliptic modulus. We denote by $\mathrm{K}(k)$ (or simply $\mathrm{K}$ ) the complete elliptic integral of the first kind associated to $k$. We denote by $\operatorname{am}(\cdot \mid k)$ the Jacobi amplitude with modulus $k$. For every complex number $\theta \in \mathbb{C}$, we define $\theta_{k}$ as

$$
\theta_{k}=\frac{2 \mathrm{~K}(k)}{\pi} \theta .
$$




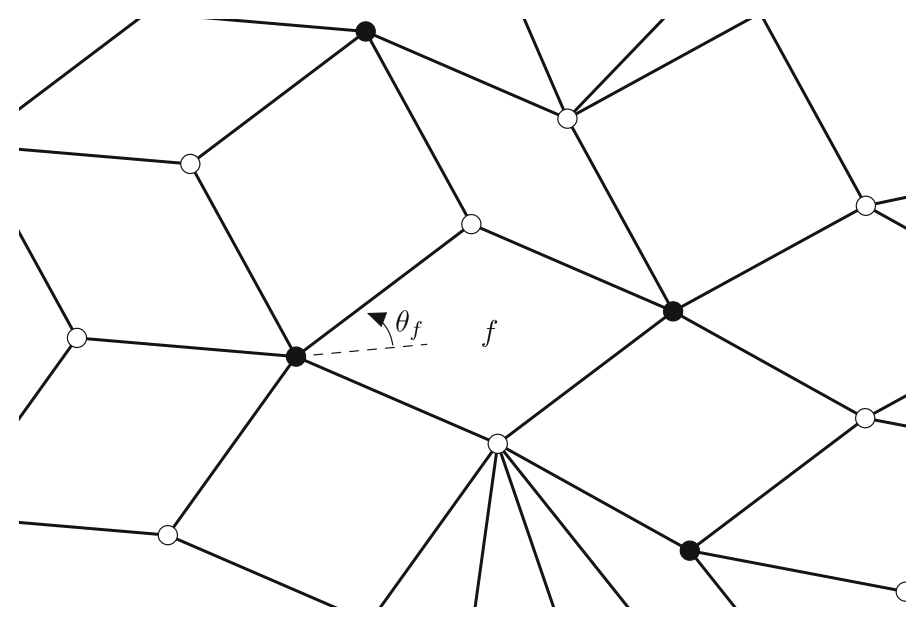

Fig. 17. A portion of a lozenge graph

Proposition 31. Let $\mathcal{Q}$ be a lozenge graph. Let $k$, l be two elliptic moduli, with $k^{2} \leq l^{2}$. For every $f \in \mathcal{F}$, let

$$
\begin{aligned}
\alpha(f) & =\operatorname{am}\left(\theta(f)_{k} \mid k\right), \\
\beta(f) & =\operatorname{am}\left(\theta(f)_{l} \mid l\right) .
\end{aligned}
$$

Then $\alpha, \beta$ satisfy (13), and the weights $X_{\alpha, \beta}$ satisfy the Yang-Baxter equations (45).

Proof. The rhombi are supposed to be nondegenerate so that $\theta_{f} \in\left(0, \frac{\pi}{2}\right)$, and for $u \in(0, \mathrm{~K}(k))$ one has am $(u \mid k) \in\left(0, \frac{\pi}{2}\right)$ (see for instance [1]). To show that (13) holds, it suffices to show that for all $\lambda \in(0,1)$,

$$
f_{\lambda}(k)=\operatorname{am}(\lambda \mathrm{K}(k) \mid k)
$$

is an increasing function of $k^{2} \in(-\infty, 1)$. This has been shown in [38] (see also [17] for a reference in English) on the domain $k^{2} \in[0,1)$, but the proof works identically for $k^{2} \in(-\infty, 1)$.

We now prove (45). It is easy to check that these equations are unchanged if we multiply the weights $d$ at every face by -1 . Simple but lengthy computations also show that they are still satisfied if we apply the duality of Proposition 12 at every face. As a result, we just have to check equations 45 for the weights (16) (we show how these weights can be transformed into $X_{\alpha, \beta}$ in the proof of Corollary 14). This holds iff the Ising models defined by $\alpha$ and $\beta$ of (46) satisfy the star-triangle relations on lozenge graphs, which is the case as shown in [12].

Remark 32. The weights of this $Z$-invariant $8 \mathrm{~V}$ model are

$$
\begin{aligned}
& A(f)=\operatorname{sn}\left(\theta(f)_{k} \mid k\right)+\operatorname{sn}\left(\theta(f)_{l} \mid l\right) \\
& B(f)=\operatorname{cn}\left(\theta(f)_{k} \mid k\right)+\operatorname{cn}\left(\theta(f)_{l} \mid l\right) \\
& C(f)=1+\operatorname{sn}\left(\theta(f)_{k} \mid k\right) \operatorname{sn}\left(\theta(f)_{l} \mid l\right)+\operatorname{cn}\left(\theta(f)_{k} \mid k\right) \operatorname{cn}\left(\theta(f)_{l} \mid l\right) \\
& D(f)=\operatorname{cn}\left(\theta(f)_{k} \mid k\right) \operatorname{sn}\left(\theta(f)_{l} \mid l\right)-\operatorname{sn}\left(\theta(f)_{k} \mid k\right) \operatorname{cn}\left(\theta(f)_{l} \mid l\right)
\end{aligned}
$$




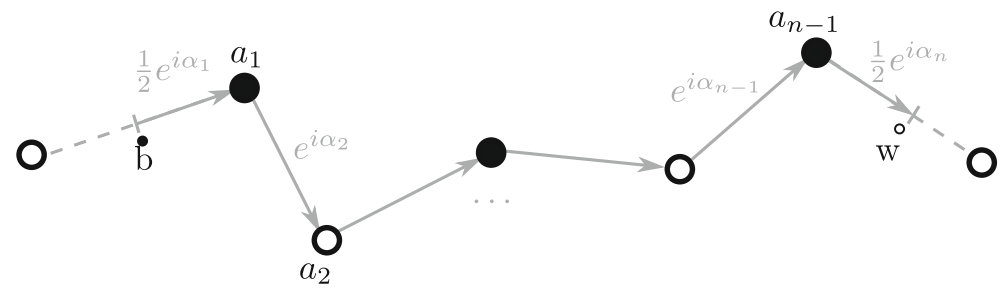

Fig. 18. A path on $\mathcal{Q}$ from b to $\mathrm{w}$

The dual modulus of $k$ is defined as $k^{\prime}=\sqrt{1-k^{2}}$. When $k^{\prime}=\frac{1}{l^{\prime}}$, (or $l=k^{*}$ in the notations of [16]), the bipartite coloring no longer matters and we recover the $Z$-invariant weights of Baxter $[5,6]$ at the free-fermion point.

When $k=l$, we get a $Z$-invariant $6 \mathrm{~V}$ model whose corresponding bipartite dimer model has been studied in [16].

From now on, we suppose that $\mathcal{Q}$ is a lozenge graph, and that two elliptic moduli $k^{2} \leq l^{2}$ are chosen. We replace the indices $\alpha, \beta$ by $k, l$, meaning that they correspond to the $\alpha, \beta$ of (46). We also slightly modify our Kasteleyn matrices by setting $\phi_{e}=\theta_{e}$ in the notations of Fig. 10. These angles also satisfy (26), (27), (28) so the results of Sect. 4 still hold.

5.3. Local expression for $K_{k, l}^{-1}$. In the case where $k=l$, we have $\alpha=\beta$ and we already know that this corresponds to a free-fermionic six-vertex model - or equivalently to dimers on a bipartite decorated graph $G^{Q}$. The operator $K_{k, k}$ can be written as

$$
K_{k, k}=i\left(\begin{array}{cc}
W^{Q} & B^{Q} \\
{ }^{t} \mathcal{K}_{k} & 0
\end{array}\right) \quad{ }_{B}^{Q}
$$

where $\mathcal{K}_{k}$ is the operator from black to white vertices, associated to the elliptic modulus $k$, defined in Section 5 of [16]; we only change notation slightly to emphasize the dependence on $k$. In the following subsection we recall the tools of $[14,16]$ that are required to compute a local formula for $\mathcal{K}_{k}^{-1}$. Our definitions differ from those of $[14,16]$ by the multiplication of the arguments by a factor $\frac{\pi}{2 \mathrm{~K}(k)}$, which is aimed at making the dependence in $k$ more apparent.

5.3.1. Inverse of $\mathcal{K}_{k}$ Let $b \in B^{T}$ and $w \in W^{T}$. We chose a path on $\mathcal{Q}$ going from the halfedge closest to $\mathrm{b}$ to the half-edge closest to $\mathrm{w}$, which we denote $\frac{1}{2} e^{i \alpha_{1}}, e^{i \alpha_{2}}, \ldots, e^{i \alpha_{n-1}}$, $\frac{1}{2} e^{i \alpha_{n}}$. We also set $a_{1}, \ldots, a_{n-1}$ to be the successive vertices of $\mathcal{Q}$ in that path. See Fig. 18. The following definitions do not depend on the choice of this path.

The discrete $k$-massive exponential function is defined in [14] as

$$
\mathrm{e}_{a_{1}, a_{n-1}}(u \mid k)=\prod_{j=2}^{n-1} i \sqrt{k^{\prime}} \operatorname{sc}\left(\left(\frac{u-\alpha_{j}}{2}\right)_{k} \mid k\right) .
$$


This is a well-defined function of the complex argument $u$. It is moreover $2 \pi$-periodic, and $2 i \pi \frac{\mathrm{K}^{\prime}}{\mathrm{K}}$-periodic when $a_{1}$ and $a_{n-1}$ are the same color (i.e. the product contains an even number of terms), $2 i \pi \frac{\mathrm{K}^{\prime}}{\mathrm{K}}$-antiperiodic otherwise.

Let also

$$
h(u \mid k)= \begin{cases}\operatorname{nc}\left(\left(\frac{u-\alpha_{1}}{2}\right)_{k} \mid k\right) \mathrm{nc}\left(\left(\frac{u-\alpha_{n}}{2}\right)_{k} \mid k\right)\left(-k^{\prime}\right) & \text { if } a_{1} \in \mathcal{B}, a_{n-1} \in \mathcal{B} \\ \operatorname{nc}\left(\left(\frac{u-\alpha_{1}}{2}\right)_{k} \mid k\right) \mathrm{dc}\left(\left(\frac{u-\alpha_{n}}{2}\right)_{k} \mid k\right)\left(-\sqrt{k^{\prime}}\right) & \text { if } a_{1} \in \mathcal{B}, a_{n-1} \in \mathcal{W} \\ \operatorname{dc}\left(\left(\frac{u-\alpha_{1}}{2}\right)_{k} \mid k\right) \mathrm{nc}\left(\left(\frac{u-\alpha_{n}}{2}\right)_{k} \mid k\right) \sqrt{k^{\prime}} & \text { if } a_{1} \in \mathcal{W}, a_{n-1} \in \mathcal{B} \\ \operatorname{dc}\left(\left(\frac{u-\alpha_{1}}{2}\right)_{k} \mid k\right) \mathrm{dc}\left(\left(\frac{u-\alpha_{n}}{2}\right)_{k} \mid k\right) & \text { if } a_{1} \in \mathcal{W}, a_{n-1} \in \mathcal{W} .\end{cases}
$$

Then the function $e^{-\frac{i}{2}\left(\alpha_{n}-\alpha_{1}\right)} h(u \mid k)$ is well defined and has the same (anti)periodicity as $e_{a_{1}, a_{n-1}}(u \mid k)$. As a result the following function is meromorphic on the torus $\mathbb{T}(k)=$ $\mathbb{C} /\left(2 \pi \mathbb{Z}+2 i \pi \frac{\mathrm{K}^{\prime}}{\mathrm{K}} \mathbb{Z}\right)$ :

$$
f_{\mathrm{b}, \mathrm{w}}(u \mid k)=e^{i \theta(\mathrm{w})} e^{-\frac{i}{2}\left(\alpha_{n}-\alpha_{1}\right)} h(u \mid k) e_{a_{1}, a_{n-1}}(u \mid k) .
$$

Its only possible poles are the $\alpha_{i}+\pi$. On can chose the paths joining $\mathrm{b}$ and w such that the angles $\alpha_{i}$ all lie in an open interval of length $\pi$. Let $\Gamma_{\mathrm{b}, \mathrm{w} \mid k}$ be a vertical contour on $\mathbb{T}(k)$ avoiding this sector.

Theorem ([16], Theorem 37). For $\mathrm{b} \in B^{T}, \mathrm{w} \in W^{T}$, let

$$
\mathcal{K}_{k}^{-1}[\mathrm{~b}, \mathrm{w}]=\frac{\mathrm{K}(k)}{2 i \pi^{2}} \int_{\Gamma_{\mathrm{b}, \mathrm{w} \mid k}} f_{\mathrm{b}, \mathrm{w} \mid k}(u) \mathrm{d} u .
$$

Then $\mathcal{K}_{k}^{-1}$ is an inverse of the operator $\mathcal{K}_{k}$. For $k \neq 0$, it is the only inverse with bounded coefficients.

5.3.2. Inverse of $K_{k, l}$ By (47), the following are inverses of $K_{k, k}$ and $K_{l, l}$ :

$$
K_{k, k}^{-1}=-i\left(\begin{array}{cc}
0 & W^{T} \\
{ }^{t} \mathcal{K}_{k}^{-1} \\
\mathcal{K}_{k}^{-1} & 0
\end{array}\right){ }_{B^{T}}^{W^{T}}, \quad K_{l, l}^{-1}=-i\left(\begin{array}{cc}
0 & { }^{t} \mathcal{K}_{l}^{-1} \\
\mathcal{K}_{l}^{-1} & 0
\end{array}\right){ }_{B^{T}}^{W^{T}} .
$$

\section{Corollary 33. The operator}

$$
K_{k, l}^{-1}=\frac{1}{2}\left((I+T) K_{k, k}^{-1}+(I-T) K_{l, l}^{-1}\right)
$$

is an inverse of $K_{k, l}$. It is the only inverse with bounded coefficients.

Its coefficients read, for $\mathrm{w}, \mathrm{w}^{\prime} \in W^{T}, \mathrm{~b}, \mathrm{~b}^{\prime} \in B^{T}$,

$$
\begin{aligned}
K_{k, l}^{-1}[\mathrm{w}, \mathrm{b}] & =\frac{-i}{2}\left(\mathcal{K}_{k}^{-1}[\mathrm{~b}, \mathrm{w}]+\mathcal{K}_{l}^{-1}[\mathrm{~b}, \mathrm{w}]\right), \\
K_{k, l}^{-1}[\mathrm{~b}, \mathrm{w}] & =\frac{-i}{2}\left(\overline{\mathcal{K}_{k}^{-1}[\mathrm{~b}, \mathrm{w}]}+\overline{\mathcal{K}_{l}^{-1}[\mathrm{~b}, \mathrm{w}]}\right), \\
K_{k, l}^{-1}\left[\mathrm{w}, \mathrm{w}^{\prime}\right] & =\frac{i e^{i \theta(\mathrm{w})}}{2}\left(\overline{\mathcal{K}_{k}^{-1}\left[\hat{\mathrm{w}}, \mathrm{w}^{\prime}\right]}-\overline{\mathcal{K}_{l}^{-1}\left[\hat{\mathrm{w}}, \mathrm{w}^{\prime}\right]}\right), \\
K_{k, l}^{-1}\left[\mathrm{~b}, \mathrm{~b}^{\prime}\right] & =\frac{i e^{-i \theta(\mathrm{b})}}{2}\left(\mathcal{K}_{k}^{-1}\left[\mathrm{~b}^{\prime}, \hat{\mathrm{b}}\right]-\mathcal{K}_{l}^{-1}\left[\mathrm{~b}^{\prime}, \hat{\mathrm{b}}\right]\right) .
\end{aligned}
$$

Proof. This is a direct consequence of (47), (48) and Theorem 23. 
5.3.3. Asymptotics of coefficients The asymptotics of the coefficients of $\mathcal{K}^{-1}$ for points $\mathrm{b}$ and $\mathrm{w}$ far away is also computed in [16]. To state the result, using the notations of Sect. 5.3.1, we also introduce the following real function:

$$
\chi(u, k)=\frac{1}{\left|a_{1}-a_{n-1}\right|} \log \left[\mathrm{e}_{a_{1}, a_{n-1}}\left(u+i \pi \frac{\mathrm{K}^{\prime}}{\mathrm{K}} \mid k\right)\right] .
$$

As stated before, the $\alpha_{i}$ can be taken in an interval of length $\pi$; let $\alpha$ be the center of this interval. According to [14], for any $k \in(0,1)$ the equation $\frac{\partial \chi}{\partial u}(u, k)=0$ has a unique solution in $\alpha+\left(-\frac{\pi}{2}, \frac{\pi}{2}\right)$. Let $u_{0}(k)$ be this solution, then $u_{0}(k)$ corresponds to a local minimum of $\chi(\cdot \mid k)$, and $\chi\left(u_{0}(k), k\right)<0$.

Theorem 34 ([16], Theorem 38). Let $\mathcal{Q}$ be a quasicrystalline planar lozenge graph, and $k \in(0,1)$. Then when $|\mathrm{b}-\mathrm{w}| \rightarrow \infty$,

$$
\mathcal{K}_{k}^{-1}[\mathrm{~b}, \mathrm{w}]=\frac{\mathrm{K}(k) e^{i \theta(\mathrm{w})} e^{-\frac{i}{2}\left(\alpha_{n}-\alpha_{1}\right)} h\left(u_{0}(k)+i \pi \frac{\mathrm{K}^{\prime}}{\mathrm{K}} \mid k\right)+o(1)}{\sqrt{2 \pi^{2}\left|a_{1}-a_{n-1}\right| \frac{\partial^{2} \chi}{\partial u^{2}}\left(u_{0}(k), k\right)}} e^{\left|a_{1}-a_{n-1}\right| \chi\left(u_{0}(k), k\right)} .
$$

The case $k=0$ can be deduced from Theorem 4.3 of [45] and corresponds to a polynomial decay of the coefficients of the inverse matrix.

To get precise asymptotics for $K_{k, l}^{-1}$, we need to compare two terms coming from $\mathcal{K}_{k}^{-1}$ and $\mathcal{K}_{l}^{-1}$. The following Lemma lets us compare the main term, $e^{\left|a_{1}-a_{n-1}\right| \chi\left(u_{0}(k), k\right)}$. The conclusion is natural, since the case $k=0$ corresponds to critical models (where the decay of correlations is polynomial), while as $k$ gets bigger the decay is exponential and should have a faster rate. Thus for $0<k<l<1$, only the term corresponding to $k$ remains in the asymptotics.

Lemma 35. The function $k \mapsto\left|\chi\left(u_{0}(k), k\right)\right|$ is increasing in $(0,1)$.

The proof can be found in Appendix B.

Remark 36. In the case of the $Z$-invariant elliptic Laplacian [14], Ising or free-fermion $6 \mathrm{~V}$ model [16], the characteristic polynomial defines a Harnack curve of genus 1 ; in fact every Harnack curve of genus 1 with a central symmetry can be obtained in this way. This means that its amoeba's complement has only one bounded component, or "oval" (see Fig. 2). The boundary of this convex oval is parametrized by functions $\chi(\cdot, k)$ for appropriate paths, and the value of $\chi\left(u_{0}(k), k\right)$ corresponds to the position of an extremal point of the oval in the path direction. Thus Lemma 35 shows that as $k$ goes from 0 to 1 , these ovals are actually included into each other. In [14] the authors show that the area of the oval grows from 0 to $\infty$, but the monotonic inclusion is new.

We can now deduce the asymptotics of coefficients for $K_{k, l}^{-1}$. There is a technical difficulty due to the fact that the prefactor $h\left(u_{0}(k)+i \pi \frac{\mathrm{K}^{\prime}}{\mathrm{K}} \mid k\right)$ in Theorem 34 can be zero. This may happen when $u_{0}(k)$ is equal to $\alpha_{1}$ or to $\alpha_{n}$, in the notation of Fig. 18. We do not expect this to happen except for a finite number of moduli $k$, but we could not get rid of this hypothesis. 
Corollary 37. Let $\mathcal{Q}$ be a quasicrystalline planar lozenge graph, and $0 \leq k<1$. We let $|\mathrm{b}-\mathrm{w}| \rightarrow \infty$; suppose that there is an $\epsilon>0$ such that $\left|u_{0}(k)-\alpha_{1}\right|>\epsilon,\left|u_{0}(k)-\alpha_{n}\right|>\epsilon$ for all b, w. Then

$$
K_{k, l}^{-1}[\mathrm{w}, \mathrm{b}]=\frac{-i \mathrm{~K}(k) e^{i \theta(\mathrm{w})} e^{-\frac{i}{2}\left(\alpha_{n}-\alpha_{1}\right)} h\left(u_{0}(k)+i \pi \frac{\mathrm{K}^{\prime}}{\mathrm{K}} \mid k\right)+o(1)}{2 \sqrt{2 \pi^{2}\left|a_{1}-a_{n-1}\right| \frac{\partial^{2} \chi}{\partial u^{2}}\left(u_{0}(k), k\right)}} e^{\left|a_{1}-a_{n-1}\right| \chi\left(u_{0}(k), k\right)} .
$$

Proof. This comes immediately from Corollary 33, Theorem 34 and Lemma 35. The fact that $h$ is bounded away from zero is a consequence of the technical hypothesis, and the fact that $\frac{\partial^{2} \chi}{\partial u^{2}}\left(u_{0}(k), k\right)$ is bounded and bounded away from zero is proven in [14].

The other coefficients of $K_{k, l}^{-1}$ can be computed in a similar way using Corollary 33, giving the same exponential behavior. When $k=0<l<1$, the decay is polynomial, so that all these models can be considered as "critical".

We conclude this part on asymptotics with the computation of a critical parameter.

Proposition 38. Let $\mathcal{Q}$ be a quasicrystalline planar lozenge graph. For any b, w, as $k \rightarrow 0$, there exists positive constants $c, C>0$ such that the exponential rate of decay $\chi\left(u_{0}(k), k\right)$ satisfies

$$
-C k^{2} \leq \chi\left(u_{0}(k), k\right) \leq-c k^{2} .
$$

Proof. In the notations of Appendix B, we showed that the minimum of $g$ is $\frac{1}{2} \log \left(k^{\prime}\right)$, so that

$$
\chi\left(u_{0}(k), k\right) \geq \frac{n-2}{2 r} \log \left(k^{\prime}\right)=\frac{n-2}{2 r} \log \left(\left(1-k^{2}\right)^{1 / 2}\right) \sim-\frac{n-2}{4 r} k^{2} .
$$

On the other hand, by Lemma 16 of [14], there exists an $\epsilon>0$ such that $\chi\left(u_{0}(k), k\right)<$ $\log \left(\sqrt{k^{\prime}}\right.$ nd $\left.(\epsilon \mid k)\right)$. As nd $(\epsilon \mid k) \rightarrow 1$ when $k \rightarrow 0$, this is equivalent to $-\frac{1}{4} k^{2}$.

5.4. Free energy and Gibbs measure. A Gibbs measure can be constructed by taking the limit of Boltzmann measures on toric graphs, i.e. to consider periodic boundary conditions. When $\mathcal{Q}$ is a $\mathbb{Z}^{2}$-periodic quadrangulation, we can define a toric exhaustion by $\mathcal{Q}_{n}=\mathcal{Q} / n \mathbb{Z}^{2}$. Using this idea and the machinery developed for finite graphs in Sect. 4, we can prove Theorem 6.

Proof of Theorem 6. The proof follows closely from the arguments of [21], see also Theorem 6 of [12]. We sketch the main steps here.

First consider the case where $\mathcal{Q}$ is $\mathbb{Z}^{2}$-periodic. We denote $\mathbb{P}_{8 V}^{n}$ the Boltzmann probability on $\mathcal{Q}_{n}$. We use Kolmogorov's extension theorem; to do so, it is sufficient to show that the right-hand side of (4) is the limit as $n \rightarrow \infty$ of the probability that $e_{1}, \ldots, e_{m} \in \tau$ in the toric graph $\mathcal{Q}_{n}$. This probability is given by (29). Thus we want to compute the limit of $\operatorname{Pf}\left(\tilde{K}_{n ; k, l}^{s, t}\right)_{V^{c}}$ (where the $n$ means that the matrix is defined on $\mathcal{Q}_{n}$ ) for any $s, t \in\{0,1\}$. When $k \neq 0$ or $(s, t) \neq(0,0)$, the matrix $\tilde{K}_{n ; k, l}^{s, t}$ is invertible and by Jacobi's identity,

$$
\operatorname{Pf}\left(\tilde{K}_{n ; k, l}^{s, t}\right)_{V^{c}}=\operatorname{Pf}\left[\left(\tilde{K}_{n ; k, l}^{s, t}\right)^{-1}\right]_{V} \operatorname{Pf}\left(\tilde{K}_{n ; k, l}^{s, t}\right) .
$$


By Lemma 20 and Theorem 26,

$$
\left(\tilde{K}_{n ; k, l}^{s, t}\right)^{-1}=\mathcal{D}^{-1} \frac{1}{2}\left[(I+T)\left(K_{n ; k, k}^{s, t}\right)^{-1}+(I-T)\left(K_{n ; l, l}^{s, t}\right)^{-1}\right] \mathcal{D} .
$$

As $n \rightarrow \infty$, the coefficients of $\left(K_{n ; k, k}^{s, t}\right)^{-1}$ tend to that of the infinite matrix $K_{k, k}^{-1}$ by the $6 \mathrm{~V}$ case [16]. Using Corollary 33 we get that the coefficients of $\left(\tilde{K}_{n ; k, l}^{s, t}\right)^{-1}$ converge to that of the infinite matrix $\tilde{K}_{k, l}^{-1}$ (where the orientation of the infinite graph is obtained by periodizing the orientation of $\left.\mathcal{Q}_{1}\right)$.

As a result, (50) implies that for $(s, t) \neq(0,0)$ or $k \neq 0$,

$$
\operatorname{Pf}\left(\tilde{K}_{n ; k, l}^{s, t}\right)_{V^{c}} \sim_{n \rightarrow \infty} \operatorname{Pf}\left[\tilde{K}_{k, l}^{-1}\right]_{V} \operatorname{Pf}\left(\tilde{K}_{n ; k, l}^{s, t}\right) .
$$

When $k=0$ and $(s, t)=(0,0)$, the generic arguments in [12] imply that

$$
\frac{\operatorname{Pf}\left(\tilde{K}_{n ; 0, l}^{0,0}\right)_{V^{c}}}{\mathcal{Z}_{8 V}\left(\mathcal{Q}_{n}, X_{0, l}\right)}=O\left(\frac{1}{n}\right) .
$$

Using these estimates, Proposition 22 implies

$$
\mathbb{P}_{8 V}^{n}\left(e_{1}, \ldots, e_{m} \in \tau\right) \sim \operatorname{Pf}\left[\tilde{K}_{k, l}^{-1}\right]_{V}\left(\frac{\prod_{f \in \mathcal{F}_{n}} C_{k, l}(f)}{2 \mathcal{Z}_{8 V}\left(\mathcal{Q}_{n}, X_{k, l}\right)} \sum_{s, t} \epsilon_{s, t} \operatorname{Pf}\left(\tilde{K}_{n ; k, l}^{s, t}\right)\right)
$$

where $\epsilon_{0,0}=-1$ and the others are 1 . By Corollary 21, the right-hand side is simply $\operatorname{Pf}\left[\tilde{K}_{k, l}^{-1}\right]_{V}=\sqrt{\operatorname{det}\left[\left(K_{k, l}^{-1}\right)_{V}\right]}$.

The non-periodic case can be deduced from the periodic case by the generic arguments of [22]. It comes from the uniqueness of an inverse of the infinite Kasteleyn matrix with bounded coefficients and the locality property of Corollary 33 .

When $\mathcal{Q}$ is $\mathbb{Z}^{2}$-periodic, the free energy is defined as

$$
F_{8 V}^{k, l}=-\lim _{n \rightarrow \infty} \frac{1}{n^{2}} \log \mathcal{Z}_{8 V}\left(\mathcal{Q}_{n}, X_{k, l}\right) .
$$

Its existence and exact value can be deduced from that of dimers [21,48], giving the following:

Proposition 39. Let $\mathcal{Q}$ be a periodic lozenge graph, and $0 \leq k<l<1$. Let $P_{k, l}^{8 V}$ be the characteristic polynomial of the $8 \mathrm{~V}$-model on the toric graph $\mathcal{Q}_{1}$. Then

$$
F_{8 V}^{k, l}=-\sum_{f \in \mathcal{F}_{1}} \log C_{k, l}(f)-\frac{1}{2} \iint_{\mathbb{T}^{2}}\left|\log P_{k, l}^{8 V}(z, w)\right| \frac{\mathrm{d} z}{2 i \pi z} \frac{\mathrm{d} w}{2 i \pi w} .
$$

Acknowledgements. I am very grateful to Cédric Boutillier and Béatrice de Tilière for the motivation of this paper, as well as their help and supervision. I also thank Béatrice de Tilière for her critical help in proving Theorem 27. I am grateful to Yacine Ikhlef for useful discussions. The author acknowledges support from the Agence Nationale de la Recherche, Grant Number ANR-18-CE40-0033 (ANR DIMERS).

Funding Open access funding provided by University of Fribourg. 
Open Access This article is licensed under a Creative Commons Attribution 4.0 International License, which permits use, sharing, adaptation, distribution and reproduction in any medium or format, as long as you give appropriate credit to the original author(s) and the source, provide a link to the Creative Commons licence, and indicate if changes were made. The images or other third party material in this article are included in the article's Creative Commons licence, unless indicated otherwise in a credit line to the material. If material is not included in the article's Creative Commons licence and your intended use is not permitted by statutory regulation or exceeds the permitted use, you will need to obtain permission directly from the copyright holder. To view a copy of this licence, visit http://creativecommons.org/licenses/by/4.0/.

Publisher's Note Springer Nature remains neutral with regard to jurisdictional claims in published maps and institutional affiliations.

\section{A. 8V-Configurations as 1-Forms}

This section aims at providing a simple algebraic framework to understand $8 \mathrm{~V}$ duality and order-disorder variables. Specifically, we write configurations as elements of certain $\mathbb{Z}_{2}$-modules, so we use additive notations; similar definitions can be found for various models, in multiplicative notation, in [27]. We do this for a quadrangulation $\mathcal{Q}$ only in the spherical case.

A.1. Setup. A spin configuration on the vertices $\mathcal{V}$ of $\mathcal{Q}$ can be seen as an element $\sigma \in \mathbb{Z}_{2}^{\mathcal{V}}$ (we will use bold notations to represent objects defined in $\mathbb{Z}_{2}$-modules). Then the spin-vertex correspondence sketched at the beginning of Sect. 3.1 can be seen as a linear map $\boldsymbol{\Phi}: \mathbb{Z}_{2}^{\mathcal{V}} \rightarrow\left(\mathbb{Z}_{2}^{2}\right)^{\mathcal{F}}$, such that for a spin configuration $\sigma=\left(\sigma_{v}\right)_{v \in \mathcal{V}}$ and a face $f \in \mathcal{F}$ with boundary vertices $b, b^{\prime} \in \mathcal{B}$ and $w, w^{\prime} \in \mathcal{W}$,

$$
\boldsymbol{\Phi}(\boldsymbol{\sigma})_{f}=\left(\sigma_{b}+\sigma_{b^{\prime}}, \sigma_{w}+\sigma_{w^{\prime}}\right) \text {. }
$$

Thus an 8V-configuration can be represented as an element $\boldsymbol{\tau}=\left(\boldsymbol{\alpha}_{f}, \boldsymbol{\beta}_{f}\right)_{f \in \mathcal{F}} \in\left(\mathbb{Z}_{2}^{2}\right)^{\mathcal{F}}$, with $\boldsymbol{\alpha}_{f}$ taking the value $\mathbf{0}$ when $\boldsymbol{\tau}_{f}$ is of type $A$ or $C$ (i.e. the black spins are equal) and $\mathbf{1}$ when it is of type $B$ or $D$, and similarly for $\boldsymbol{\beta}_{f}$ and white spins. Note that this characterizes the $8 \mathrm{~V}$ configuration up to global complement, so that this setup is only relevant in the zero field case.

Thus we define the set of $8 \mathrm{~V}$-configurations as $\boldsymbol{H}=\operatorname{Im} \boldsymbol{\Phi}$, which is a strict subset of $\left(\mathbb{Z}_{2}^{2}\right)^{\mathcal{F}}$. Also note that if $\boldsymbol{\tau}=\left(\boldsymbol{\alpha}_{f}, \boldsymbol{\beta}_{f}\right)_{f \in \mathcal{F}} \in \boldsymbol{H}$, then for any $b \in \mathcal{B}, \sum_{f \sim b} \boldsymbol{\beta}_{f}=\mathbf{0}$ where the sum is over all faces adjacent to $b$. Similarly, if $w \in \mathcal{W}, \sum_{f \sim w} \boldsymbol{\alpha}_{f}=\mathbf{0}$. This motivates the definition of $\boldsymbol{\Psi}:\left(\mathbb{Z}_{2}^{2}\right)^{\mathcal{F}} \rightarrow \mathbb{Z}_{2}^{\mathcal{V}}$, such that if $\boldsymbol{\tau}=\left(\boldsymbol{\alpha}_{f}, \boldsymbol{\beta}_{f}\right)_{f \in \mathcal{F}} \in\left(\mathbb{Z}_{2}^{2}\right)^{\mathcal{F}}$,

$$
\boldsymbol{\Psi}(\boldsymbol{\tau})_{x}= \begin{cases}\sum_{f \sim x} \boldsymbol{\beta}_{f} & \text { if } x \in \mathcal{B}, \\ \sum_{f \sim x} \boldsymbol{\alpha}_{f} & \text { if } x \in \mathcal{W} .\end{cases}
$$

The applications $\boldsymbol{\Phi}$ and $\boldsymbol{\Psi}$ can be considered as dual of each other. To do so, we equip $\left(\mathbb{Z}_{2}^{2}\right)^{\mathcal{F}}$ with the symplectic form $\langle\cdot \mid \cdot\rangle$

$$
\left\langle\boldsymbol{\tau} \mid \boldsymbol{\tau}^{\prime}\right\rangle=\sum_{f \in F} \boldsymbol{\alpha}_{f} \boldsymbol{\beta}_{f}^{\prime}+\boldsymbol{\alpha}_{f}^{\prime} \boldsymbol{\beta}_{f},
$$

and $\mathbb{Z}_{2}^{\mathcal{V}}$ with the canonical bilinear symmetric form $(\cdot, \cdot)$

$$
\left(\sigma, \sigma^{\prime}\right)=\sum_{v \in V} \sigma_{v} \sigma_{v}^{\prime} .
$$




\section{Proposition 40.}

1. The applications $\boldsymbol{\Psi}$ and $\boldsymbol{\Phi}$ are dual of each other, meaning that for any $\sigma \in \mathbb{Z}_{2}^{\mathcal{V}}$ and $\boldsymbol{\tau} \in\left(\mathbb{Z}_{2}^{2}\right)^{\mathcal{F}}$,

$$
\langle\boldsymbol{\Phi} \sigma \mid \tau\rangle=(\sigma, \Psi \tau) .
$$

2. $\boldsymbol{H}=\operatorname{Im} \boldsymbol{\Phi}=\operatorname{ker} \boldsymbol{\Psi}$. In other words, the following sequence is exact

$$
\mathbb{Z}_{2}^{\mathcal{V}} \stackrel{\Phi}{\rightarrow}\left(\mathbb{Z}_{2}^{2}\right)^{\mathcal{F}} \stackrel{\Psi}{\rightarrow} \mathbb{Z}_{2}^{\mathcal{V}}
$$

3. $\boldsymbol{H}=\boldsymbol{H}^{\perp}$.

Proof. Let $\boldsymbol{\sigma}=\left(\boldsymbol{\sigma}_{v}\right)_{v \in \mathcal{V}}$ and $\boldsymbol{\tau}=\left(\boldsymbol{\alpha}_{f}, \boldsymbol{\beta}_{f}\right)_{f \in \mathcal{F}}$. By linearity, it is enough to prove (52) when $\sigma, \boldsymbol{\tau}$ are elements of the canonical basis, i.e. when $\sigma_{v}$ is $\mathbf{0}$ for all vertices but one, and $\boldsymbol{\alpha}_{f}, \boldsymbol{\beta}_{f}$ are all $\boldsymbol{0}$ except one.

If $\boldsymbol{\sigma}_{b}$ is $\mathbf{1}$ for one black vertex $b \in \mathcal{B}$ and $\mathbf{0}$ for all other vertices, then $\boldsymbol{\Phi} \boldsymbol{\sigma}$ is $(\mathbf{1}, \mathbf{0})$ on faces adjacent to $b$ and $(\mathbf{0 ,}, \mathbf{0})$ otherwise. Two cases may appear:

- if $\boldsymbol{\alpha}_{f}=\mathbf{1}$ at some face $f$ and all the other components of $\boldsymbol{\tau}$ are $\mathbf{0}$, then $\boldsymbol{\Psi} \boldsymbol{\tau}$ is $\mathbf{1}$ on the white vertices of $f$ and $\mathbf{0}$ everywhere else, and we have

$$
\langle\boldsymbol{\Phi} \sigma \mid \boldsymbol{\tau}\rangle=(\boldsymbol{\sigma}, \boldsymbol{\Psi} \boldsymbol{\tau})=\mathbf{0} .
$$

- if $\boldsymbol{\beta}_{f}=\mathbf{1}$ at some face $f$ and all the other components of $\boldsymbol{\tau}$ are $\mathbf{0}$, then $\boldsymbol{\Psi} \boldsymbol{\tau}$ is $\mathbf{1}$ on the black vertices of $f$ and $\mathbf{0}$ everywhere else, and we have

$$
\langle\boldsymbol{\Phi} \boldsymbol{\sigma} \mid \boldsymbol{\tau}\rangle=(\boldsymbol{\sigma}, \boldsymbol{\Psi} \boldsymbol{\tau})= \begin{cases}\mathbf{1} & \text { if } u \text { is a vertex of } f \\ \mathbf{0} & \text { otherwise }\end{cases}
$$

The case where $\boldsymbol{\sigma}_{w}$ is $\mathbf{1}$ at a white vertex $w \in W$ and $\mathbf{0}$ elsewhere is similar. This proves 1 .

We now prove 2 . We already know that $\operatorname{Im} \boldsymbol{\Phi} \subset$ ker $\boldsymbol{\Psi}$. Let us show that they have the same dimension.

- The kernel of $\boldsymbol{\Phi}$ is clearly composed of elements of $\mathbb{Z}_{2}^{\mathcal{V}}$ constant on $\mathcal{B}$ and constant on $\mathcal{W}$, so it has dimension 2. By the rank-nullity theorem, $\operatorname{Im} \Phi$ has dimension $|\mathcal{V}|-2$.

- The applications $\boldsymbol{\Phi}$ and $\boldsymbol{\Psi}$ are dual of each other so they have the same rank. By the rank-nullity theorem, $\operatorname{ker} \Psi$ has dimension $2|\mathcal{F}|-|\mathcal{V}|+2$.

- We have Euler's formula $|\mathcal{V}|-|\mathcal{E}|+|\mathcal{F}|=2$, and the graph is a quadrangulation so $4|\mathcal{F}|=2|\mathcal{E}|$. Combining these gives $|\mathcal{V}|-2=2|\mathcal{F}|-|\mathcal{V}|+2$ as needed.

Since $\boldsymbol{\Phi}$ and $\boldsymbol{\Psi}$ are dual of each other, $\operatorname{Im} \boldsymbol{\Phi}=(\operatorname{ker} \boldsymbol{\Psi})^{\perp}$ and 3 is obvious from 2 .

\section{Remark 41.}

- It is clear now that we are working with an avatar of discrete Hodge theory. The applications $\boldsymbol{\Phi}$ and $\boldsymbol{\Psi}$ are in fact the $d$ applications defined by Mercat for the double of a chain complex [57]. For that reason, we will now simply denote the sequence (53) as

$$
\mathbb{Z}_{2}^{\mathcal{V}} \stackrel{\boldsymbol{d}}{\rightarrow}\left(\mathbb{Z}_{2}^{2}\right)^{\mathcal{F}} \stackrel{\boldsymbol{d}}{\rightarrow} \mathbb{Z}_{2}^{\mathcal{V}}
$$

so, for instance, an $8 \mathrm{~V}$ configuration is a closed 1-form (i.e. a $\boldsymbol{\tau} \in\left(\mathbb{Z}_{2}^{2}\right)^{\mathcal{F}}$ s.t. $\boldsymbol{d} \boldsymbol{\tau}=\mathbf{0}$ ). 
- The elements of $\left(\mathbb{Z}_{2}^{2}\right)^{\mathcal{F}} \backslash \boldsymbol{H}$ do not correspond to 8V-configurations, but can be thought of as configurations with defects. More precisely, if $\boldsymbol{d} \boldsymbol{\tau}=\mathbf{1}_{B_{1} \cup W_{1}}$, with $B_{1} \subset \mathcal{B}$ and $W_{1} \subset \mathcal{W}$, then $B_{1}$ and $W_{1}$ have to be of even cardinality, and $\boldsymbol{\tau}$ corresponds to the disordered configurations of [26] mentioned in 2.4.2. We will alternatively denote $\boldsymbol{d} \boldsymbol{\tau}=B_{1} \cup W_{1}$.

- Properties similar to Proposition 40 might hold when $\mathcal{Q}$ is not a quadrangulation of the sphere but of the torus, or other surfaces. These are beyond the scope of the present paper.

A.2. Fourier transform. Let $g:\left(\mathbb{Z}_{2}^{2}\right)^{\mathcal{F}} \rightarrow \mathbb{C}$. We define its Fourier transform $\hat{g}:$ $\left(\mathbb{Z}_{2}^{2}\right)^{\mathcal{F}} \rightarrow \mathbb{C}$ by

$$
\hat{g}(\boldsymbol{\tau})=2^{-|\mathcal{F}|} \sum_{\boldsymbol{\tau}^{\prime} \in\left(\mathbb{Z}_{2}^{2}\right)^{\mathcal{F}}}(-1)^{\left\langle\boldsymbol{\tau} \mid \boldsymbol{\tau}^{\prime}\right\rangle} g\left(\boldsymbol{\tau}^{\prime}\right)
$$

The normalization is such that we have the Inverse Fourier transform formula is $\hat{\hat{g}}=g$.

Another important formula is Poisson's summation identity. For any subspace $\boldsymbol{F} \subset$ $\left(\mathbb{Z}_{2}^{2}\right)^{\mathcal{F}}$,

$$
\sum_{\boldsymbol{\tau} \in \boldsymbol{F}} g(\boldsymbol{\tau})=\sum_{\boldsymbol{\tau} \in \boldsymbol{F}^{\perp}} \hat{g}(\boldsymbol{\tau}) .
$$

Example 42. For $8 \mathrm{~V}$ weights $X: \mathcal{F} \rightarrow \mathbb{R}^{4}$, the weight function $w_{8 \mathrm{~V}}$ that we defined for $8 \mathrm{~V}$-configuration (5) can be extended to a function on $\left(\mathbb{Z}_{2}^{2}\right)^{\mathcal{F}}$. Then it is easy to check that its Fourier transform is actually the weight function for the dual weights $\hat{X}$ (17). Then Poisson's summation identity applied to $\boldsymbol{H}$, given that $\boldsymbol{H}=\boldsymbol{H}^{\perp}$, becomes

$$
\sum_{\boldsymbol{\tau} \in \boldsymbol{H}} w_{8 V}(\boldsymbol{\tau})=\sum_{\boldsymbol{\tau} \in \boldsymbol{H}} \hat{w}_{8 V}(\boldsymbol{\tau})
$$

which is the duality relation for partition functions (18).

A.3. Correlators. We now describe how correlators of Definition 9 fit into this description. In the absence of disorder, the order variables $\sigma\left(B_{0}\right) \sigma\left(W_{0}\right)$ correspond to a random variable taking value 1 (resp. -1 ) when there is an even (resp. odd) number of edges in $\tau$ between the $B_{0}, W_{0}$ joined pairwise. If we fix paths $\gamma_{B_{0}}, \gamma_{W_{0}}$, and if $\boldsymbol{\tau}=\left(\boldsymbol{\alpha}_{f}, \boldsymbol{\beta}_{f}\right)_{f \in \mathcal{F}}$, this is equivalent to considering

$$
\prod_{f \in \gamma_{B_{0}}}(-1)^{\boldsymbol{\alpha}_{f}} \prod_{f \in \gamma_{W_{0}}}(-1)^{\boldsymbol{\beta}_{f}} .
$$

If we define $\boldsymbol{\tau}_{\gamma}=\left(\mathbf{1}_{\gamma_{W_{0}}}, \mathbf{1}_{\gamma_{B_{0}}}\right)$ (where the paths are identified with subsets of $\mathcal{F}$ ), then this quantity is exactly $(-1)^{\left\langle\boldsymbol{\tau}_{\gamma} \mid \boldsymbol{\tau}\right\rangle}$. On the other hand, disorder variables at $B_{1}, W_{1}$ correspond to configurations $\boldsymbol{\tau}$ with $\boldsymbol{d} \boldsymbol{\tau}=B_{1} \cup W_{1}$. Thus we have:

$$
\left\langle\sigma\left(B_{0}\right) \sigma\left(W_{0}\right) \mu\left(B_{1}\right) \mu\left(W_{1}\right)\right\rangle_{X, \gamma}^{8 V}=2 \sum_{\substack{\boldsymbol{\tau} \text { s.t. } \\ \boldsymbol{d}=B_{1} \cup W_{1}}}(-1)^{\left\langle\boldsymbol{\tau}_{\gamma} \mid \boldsymbol{\tau}\right\rangle} w_{8 V}(\boldsymbol{\tau}) .
$$


The factor 2 comes from the fact that the representation of $8 \mathrm{~V}$-configurations in $\left(\mathbb{Z}_{2}^{2}\right)^{\mathcal{F}}$ is two-to-one.

Proof of Theorem 3. Our goal is to prove that for any $8 \mathrm{~V}$-configuration $\tau \in \Omega(\mathcal{Q})$,

$$
\mathbb{P}_{8 V}\left(\tau_{\alpha, \beta} \oplus \tau_{\alpha^{\prime}, \beta^{\prime}}=\tau\right)=\mathbb{P}_{8 V}\left(\tau_{\alpha, \beta^{\prime}} \oplus \tau_{\alpha^{\prime}, \beta}=\tau\right)
$$

By definition of Boltzmann probabilities, this is equivalent to (we indicate the dependence of $w_{8 V}$ in the $\alpha, \beta$ variables):

$$
\sum_{\substack{\tau_{\alpha, \beta}, \tau_{\alpha^{\prime}, \beta^{\prime}} \text { s.t. } \\ \tau_{\alpha, \beta} \oplus \tau_{\alpha^{\prime}, \beta^{\prime}}=\tau}} \frac{w_{8 V}^{\alpha, \beta}\left(\tau_{\alpha, \beta}\right)}{\mathcal{Z}_{8 V}\left(\mathcal{Q}, X_{\alpha, \beta}\right)} \frac{w_{8 V}^{\alpha^{\prime}, \beta^{\prime}}\left(\tau_{\alpha^{\prime}, \beta^{\prime}}\right)}{\mathcal{Z}_{8 V}\left(\mathcal{Q}, X_{\alpha^{\prime}, \beta^{\prime}}\right)}=\sum_{\substack{\tau_{\alpha, \beta^{\prime}}, \tau_{\alpha^{\prime}, \beta} \text { s.t. } \\ \tau_{\alpha, \beta^{\prime}} \oplus \tau_{\alpha^{\prime}, \beta}=\tau}} \frac{w_{8 V}^{\alpha, \beta^{\prime}}\left(\tau_{\alpha, \beta^{\prime}}\right)}{\mathcal{Z}_{8 V}\left(\mathcal{Q}, X_{\alpha, \beta^{\prime}}\right)} \frac{w_{8 V}^{\alpha^{\prime}, \beta}\left(\tau_{\alpha^{\prime}, \beta}\right)}{\mathcal{Z}_{8 V}\left(\mathcal{Q}, X_{\alpha^{\prime}, \beta}\right)} .
$$

We already know that the product of partition functions are proportional with a factor $c_{1}$ (22), so we just have to show that

$$
\sum_{\substack{\tau_{\alpha, \beta}, \tau_{\alpha^{\prime}, \beta^{\prime}} \text { s.t. } \\ \tau_{\alpha, \beta} \oplus \tau_{\alpha^{\prime}, \beta^{\prime}}=\tau}} w_{8 V}^{\alpha, \beta}\left(\tau_{\alpha, \beta}\right) w_{8 V}^{\alpha^{\prime}, \beta^{\prime}}\left(\tau_{\alpha^{\prime}, \beta^{\prime}}\right)=c_{1} \sum_{\substack{\tau_{\alpha, \beta^{\prime}}, \tau_{\alpha^{\prime}, \beta} \text { s.t. } \\ \tau_{\alpha, \beta^{\prime}} \oplus \tau_{\alpha^{\prime}, \beta}=\tau}} w_{8 V}^{\alpha, \beta^{\prime}}\left(\tau_{\alpha, \beta^{\prime}}\right) w_{8 V}^{\alpha^{\prime}, \beta}\left(\tau_{\alpha^{\prime}, \beta}\right)(54)
$$

To prove (54), we first rewrite the correlators of Theorem 15 in the formalism of 1 -forms. In the particular case $B=B_{0}=B_{0}^{\prime}, W=W_{0}=W_{0}^{\prime}$ and $B_{1}=B_{1}^{\prime}=W_{1}=$ $W_{1}^{\prime}=\emptyset$, let $\boldsymbol{\tau}_{\gamma}=\left(\mathbf{1}_{\gamma_{W}}, \mathbf{1}_{\gamma_{B}}\right)$, then (21) reads

$$
\sum_{\boldsymbol{\tau}^{\prime}, \boldsymbol{\tau}^{\prime \prime} \in \boldsymbol{H}}(-1)^{\left\langle\boldsymbol{\tau}^{\prime}+\boldsymbol{\tau}^{\prime \prime} \mid \boldsymbol{\tau}_{\gamma}\right\rangle} w_{8 V}^{\alpha, \beta}\left(\boldsymbol{\tau}^{\prime}\right) w_{8 V}^{\alpha^{\prime}, \beta^{\prime}}\left(\boldsymbol{\tau}^{\prime \prime}\right)=c_{1} \sum_{\boldsymbol{\tau}^{\prime}, \boldsymbol{\tau}^{\prime \prime} \in \boldsymbol{H}}(-1)^{\left\langle\boldsymbol{\tau}^{\prime}+\boldsymbol{\tau}^{\prime \prime} \mid \boldsymbol{\tau}_{\gamma}\right\rangle} w_{8 V}^{\alpha, \beta^{\prime}}\left(\boldsymbol{\tau}^{\prime}\right) \boldsymbol{w}_{8 V}^{\alpha^{\prime}, \beta}\left(\boldsymbol{\tau}^{\prime \prime}\right) \text {. }
$$

Reordering these sums according to $\boldsymbol{\tau}=\boldsymbol{\tau}^{\prime}+\boldsymbol{\tau}^{\prime \prime}$ gives

$$
\begin{aligned}
& \sum_{\boldsymbol{\tau} \in\left(\mathbb{Z}_{2}^{2}\right)}(-1)^{\left\langle\mathcal{F} \mid \boldsymbol{\tau}_{\gamma}\right\rangle} \sum_{\substack{\boldsymbol{\tau}^{\prime}, \boldsymbol{\tau}^{\prime \prime} \in \boldsymbol{H} \\
\boldsymbol{\tau}^{\prime}+\boldsymbol{\tau}^{\prime \prime}=\boldsymbol{\tau}}} w_{8 V}^{\alpha, \beta}\left(\boldsymbol{\tau}^{\prime}\right) \boldsymbol{w}_{8 V}^{\alpha^{\prime}, \beta^{\prime}}\left(\boldsymbol{\tau}^{\prime \prime}\right) \\
& =c_{1} \sum_{\boldsymbol{\tau} \in\left(\mathbb{Z}_{2}^{2}\right)}(-1)^{\left\langle\boldsymbol{\tau} \mid \boldsymbol{\tau}_{\gamma}\right\rangle} \sum_{\substack{\boldsymbol{\tau}^{\prime}, \boldsymbol{\tau}^{\prime \prime} \in \boldsymbol{H} \\
\boldsymbol{\tau}^{\prime}+\boldsymbol{\tau}^{\prime \prime}=\boldsymbol{\tau}}} w_{8 V}^{\alpha, \beta^{\prime}}\left(\boldsymbol{\tau}^{\prime}\right) \boldsymbol{w}_{8 V}^{\alpha^{\prime}, \beta}\left(\boldsymbol{\tau}^{\prime \prime}\right) .
\end{aligned}
$$

Note that we always have $\boldsymbol{\tau}=\boldsymbol{\tau}^{\prime}+\boldsymbol{\tau}^{\prime \prime} \in \boldsymbol{H}$, so when $\boldsymbol{\tau} \notin \boldsymbol{H}$ the inner sum is empty. We rewrite this as

$$
\sum_{\boldsymbol{\tau} \in\left(\mathbb{Z}_{2}^{2}\right)^{\mathcal{F}}}(-1)^{\left\langle\boldsymbol{\tau} \mid \boldsymbol{\tau}_{\gamma}\right\rangle} f(\boldsymbol{\tau})=0
$$

where

$$
f(\boldsymbol{\tau})=\sum_{\substack{\boldsymbol{\tau}^{\prime}, \boldsymbol{\tau}^{\prime \prime} \in \boldsymbol{H} \\ \boldsymbol{\tau}^{\prime}+\boldsymbol{\tau}^{\prime \prime}=\boldsymbol{\tau} . t}} w_{8 V}^{\alpha, \beta}\left(\boldsymbol{\tau}^{\prime}\right) w_{8 V}^{\alpha^{\prime}, \beta^{\prime}}\left(\boldsymbol{\tau}^{\prime \prime}\right)-c_{1} \sum_{\substack{\boldsymbol{\tau}^{\prime}, \boldsymbol{\tau}^{\prime \prime} \in \boldsymbol{H} \text { s.t } \\ \boldsymbol{\tau}^{\prime}+\boldsymbol{\tau}^{\prime \prime}=\boldsymbol{\tau}}} w_{8 V}^{\alpha, \beta^{\prime}}\left(\boldsymbol{\tau}^{\prime}\right) w_{8 V}^{\alpha^{\prime}, \beta}\left(\boldsymbol{\tau}^{\prime \prime}\right) .
$$

In other words, we have $\hat{f}\left(\boldsymbol{\tau}_{\gamma}\right)=0$. This is true for any $B, W$ and paths $\gamma$ joining them pairwise. Conversely, any element $\boldsymbol{\tau} \in\left(\mathbb{Z}_{2}^{2}\right)^{\mathcal{F}}$ can be considered as such a $\boldsymbol{\tau}_{\gamma}$ - namely, if $\boldsymbol{d} \boldsymbol{\tau}=B \cup W$, then $\boldsymbol{\tau}=\left(\mathbf{1}_{\gamma_{W}}, \mathbf{1}_{\gamma_{B}}\right)$ for some paths $\gamma_{B}, \gamma_{W}$ that satisfy the hypothesis of Theorem 15 . This means that $\hat{f}$ is actually the null function, and by injectivity of the Fourier transform, so is $f$. This proves (54). 
Remark 43. In the previous proof, if we let $B_{1}, B_{1}^{\prime}, W_{1}, W_{1}^{\prime}$ be any even subsets of black and white vertices of $\mathcal{Q}$, we get

$$
\begin{aligned}
& \sum_{\substack{\boldsymbol{\tau}^{\prime}, \boldsymbol{\tau}^{\prime \prime} \in\left(\mathbb{Z}_{2}^{2}\right) \\
\boldsymbol{\tau}^{\prime}+\boldsymbol{\tau}^{\prime \prime}=\boldsymbol{\tau} \\
\boldsymbol{d} \boldsymbol{\tau}^{\prime}=B_{1} \cup W_{1} \\
\boldsymbol{d} \boldsymbol{\tau}^{\prime \prime}=B_{1}^{\prime} \cup W_{1}^{\prime}}} \frac{w_{8 V}^{\alpha, \beta}\left(\boldsymbol{\tau}^{\prime}\right)}{\mathcal{Z}_{8 V}\left(\mathcal{Q}, X_{\alpha, \beta}\right)} \frac{w_{8 V}^{\alpha^{\prime}, \beta^{\prime}}\left(\boldsymbol{\tau}^{\prime \prime}\right)}{\mathcal{Z}_{8 V}\left(\mathcal{Q}, X_{\alpha^{\prime}, \beta^{\prime}}\right)} \\
& =\sum_{\substack{\boldsymbol{\tau}^{\prime}, \boldsymbol{\tau}^{\prime \prime} \in\left(\mathbb{Z}_{2}^{2}\right)^{\mathcal{F}} \\
\boldsymbol{\tau}^{\prime}+\boldsymbol{\tau}^{\prime \prime}=\boldsymbol{\tau} \\
\boldsymbol{d} \boldsymbol{\tau}^{\prime}=B_{1}^{\prime} \cup W_{1} \\
\boldsymbol{d} \boldsymbol{\tau}^{\prime \prime}=B_{1} \cup W_{1}^{\prime}}} \frac{w_{8 V}^{\alpha, \beta^{\prime}}\left(\boldsymbol{\tau}^{\prime}\right)}{\mathcal{Z}_{8 V}\left(\mathcal{Q}, X_{\alpha, \beta^{\prime}}\right)} \frac{w_{8 V}^{\alpha^{\prime}, \beta}\left(\boldsymbol{\tau}^{\prime \prime}\right)}{\mathcal{Z}_{8 V}\left(\mathcal{Q}, X_{\alpha^{\prime}, \beta}\right)}
\end{aligned}
$$

which expresses a coupling for the XOR of 8V-configurations with disorder.

\section{B. Proof of Lemma 35}

By rotating the graph, we can suppose that $\alpha=0$, i.e. the angles $\alpha_{i}$ and $u_{0}(k)$ all lie in $\left(-\frac{\pi}{2}, \frac{\pi}{2}\right)$. We also fix a $k \in(0,1)$ and suppose that $u_{0}(k) \geq 0$, the other case being symmetric.

Using the chain rule we have

$$
\frac{\mathrm{d}}{\mathrm{d} k} \chi\left(u_{0}(k), k\right)=\frac{\mathrm{d}}{\mathrm{d} k} u_{0}(k) \frac{\partial \chi}{\partial u}\left(u_{0}(k), k\right)+\frac{\partial \chi}{\partial k}\left(u_{0}(k), k\right) .
$$

By definition of $u_{0}(k)$ the first term of the sum is null so we just have to show that $\frac{\partial \chi}{\partial k}$ is negative at $\left(u_{0}(k), k\right)$.

We denote $r=\left|a_{1}-a_{n-1}\right|$; this does not depend on $k$. By using the change of arguments in Jacobi elliptic functions (see Table 16.8 in [1]),

$$
\chi(u, k)=\frac{1}{r} \sum_{j=2}^{n-1} \log \left[\sqrt{k^{\prime}} \mathrm{nd}\left(\left(\frac{u-\alpha_{j}}{2}\right)_{k} \mid k\right)\right] .
$$

Let

$$
g(u, k)=\log \left[\sqrt{k^{\prime}} \text { nd }\left(\left(\frac{u}{2}\right)_{k} \mid k\right)\right] .
$$

By the properties of the function nd $(\cdot, k)$ (see 16.2 in [1]), for any $k \in(0,1), g(\cdot, k)$ is decreasing on $[-\pi, 0]$ and increasing on $[0, \pi]$. Its minimum is $g(0, k)=\frac{1}{2} \log \left(k^{\prime}\right)<0$. As a result, if all the angles $\alpha_{j}$ are equal, then $u_{0}(k)$ has the same value and $\chi\left(u_{0}(k), k\right)=$ $\frac{n-2}{2 r} \log \left(k^{\prime}\right)$, which is indeed a decreasing function of $k$. We now suppose that the $\alpha_{j}$ are not all equal. We need some extra properties on $g$.

Lemma 44. For all $k \in(0,1)$,

$$
\text { 1. } g(-u, k)=g(u, k) \text { and } g(\pi-u, k)=-g(u, k) \text {. }
$$


2. $\frac{\partial g}{\partial k}(u, k)$ is a strictly decreasing function of $u$ on $[-\pi, 0]$, and strictly increasing on $[0, \pi]$. It is zero at $u= \pm \frac{\pi}{2}$.

Lemma 45. We have the following inequality of cardinals:

$$
\#\left\{j \in[2, n-1] \mid \alpha_{j}<u_{0}(k)-\frac{\pi}{2}\right\}<\#\left\{j \in[2, n-1] \mid \alpha_{j}>u_{0}(k)\right\} .
$$

We prove these two Lemmas later, and first show how they imply Lemma 35. By differentiation of (55), for $u \in\left[0, \frac{\pi}{2}\right.$ ) we have (using Lemma 44 to remove possible terms equal to zero):

$$
\begin{aligned}
r \frac{\partial \chi}{\partial k}(u, k) & =\sum_{j=2}^{n-1} \frac{\partial g}{\partial k}\left(u-\alpha_{j}, k\right) \\
& =\sum_{j \mid \alpha_{j}<u-\frac{\pi}{2}} \frac{\partial g}{\partial k}\left(u-\alpha_{j}, k\right)+\sum_{j \mid u-\frac{\pi}{2}<\alpha_{j} \leq u} \frac{\partial g}{\partial k}\left(u-\alpha_{j}, k\right)+\sum_{j \mid u<\alpha_{j}} \frac{\partial g}{\partial k}\left(u-\alpha_{j}, k\right)
\end{aligned}
$$

By Lemma 44, the terms in the first sum are positive while those in the second an third sums are negative. We show that for $u=u_{0}(k)$, the first sum is, in absolute value, smaller than the third one, which is enough to conclude.

For the first sum, if $-\frac{\pi}{2}<\alpha_{j}<u-\frac{\pi}{2}$ then $\frac{\pi}{2}<u-\alpha_{j}<u+\frac{\pi}{2}$ and by Lemma 44,

$$
0<\frac{\partial g}{\partial k}\left(u-\alpha_{j}, k\right)<\frac{\partial g}{\partial k}\left(u+\frac{\pi}{2}, k\right) .
$$

Thus the first sum $S_{1}$ in (56) satisfies

$$
0 \leq S_{1} \leq\left(\frac{\partial g}{\partial k}\left(u+\frac{\pi}{2}, k\right)\right) \#\left\{j \in[2, n-1] \mid \alpha_{j}<u-\frac{\pi}{2}\right\} .
$$

Similarly, for the third sum $S_{3}$, we have

$$
S_{3} \leq\left(\frac{\partial g}{\partial k}\left(u-\frac{\pi}{2}, k\right)\right) \#\left\{j \in[2, n-1] \mid \alpha_{j}>u\right\}<0 .
$$

By Lemma 44, $g\left(u+\frac{\pi}{2}, k\right)=-g\left(u-\frac{\pi}{2}, k\right)>0$, and by differentiating the same symmetry holds for $\frac{\partial g}{\partial k}$. Hence (58) becomes

$$
\left|S_{3}\right| \geq\left(\frac{\partial g}{\partial k}\left(u+\frac{\pi}{2}, k\right)\right) \#\left\{j \in[2, n-1] \mid \alpha_{j}>u\right\} .
$$

Using (59), (57) and Lemma 45 we see that for $u=u_{0}(k),\left|S_{3}\right|>S_{1}$ as needed.

Proof of Lemma 44. The first point is a direct consequence of the change of arguments in elliptic functions, see Table 16.8 in [1].

For the second point, first notice that for all $k$, using Table 16.5 in $[1], g\left(\frac{\pi}{2}, k\right)=1$ so $\frac{\partial g}{\partial k}\left(\frac{\pi}{2}, k\right)=0$. Using the symmetries of the first point of the Lemma, it remains to check that $\frac{\partial g}{\partial k}(u, k)$ is a strictly increasing function of $u$ on $\left[0, \frac{\pi}{2}\right]$. 
Using the derivatives of elliptic functions with respect to $u$ and $k$ (see Sections 2.5 and 3.10 in [52]), and setting $v=\left(\frac{u}{2}\right)_{k}$, we get

$$
\frac{\partial g}{\partial k}(u, k)=-\frac{k}{2 k^{\prime 2}}+\frac{k}{k^{\prime 2}}\left(\frac{v}{\mathrm{~K}(k)} \mathrm{E}(k)-\mathrm{E}(v, k)+\frac{\mathrm{sndn}}{\mathrm{cn}}(v \mid k)\right) \frac{\operatorname{sn} \mathrm{cn}}{\mathrm{dn}}(v \mid k)
$$

where $\mathrm{E}$ is the elliptic integral of the second kind:

$$
\begin{aligned}
\mathrm{E}(v, k) & =\int_{0}^{v} \mathrm{dn}^{2}(t \mid k) \mathrm{d} t, \\
\mathrm{E}(k) & =\mathrm{E}(\mathrm{K}(k), k) .
\end{aligned}
$$

As $v=\frac{\mathrm{K}(k)}{\pi} u$, it is sufficient to prove that the right-hand side of (60) is a strictly increasing function of $v$ on $\left[0, \frac{\mathrm{K}(k)}{2}\right]$. On that interval,

- $v \mapsto \frac{v}{\mathrm{~K}(k)} \mathrm{E}(k)-\mathrm{E}(v, k)+\frac{\mathrm{sndn}}{\mathrm{cn}}(v \mid k)$ is strictly increasing because its derivative in $v$ is (using Section 2.5 in [52])

$$
\frac{\mathrm{E}(k)}{\mathrm{K}(k)}+k^{\prime 2} \operatorname{sc}(v \mid k)>0
$$

- $v \mapsto \frac{\mathrm{sncn}}{\mathrm{dn}}(v \mid k)$ is strictly increasing because, using the ascending Landen transform $\tilde{k}=\frac{1-k^{\prime}}{1+k^{\prime}}($ see 16.14 .1 in [1]), this is equal to

$$
\frac{1+\tilde{k}}{2} \operatorname{sn}\left(2 \frac{\mathrm{K}(\tilde{k})}{\mathrm{K}(k)} v \mid \tilde{k}\right)
$$

and $\operatorname{sn}(\cdot \mid \tilde{k})$ is strictly increasing on $[0, \mathrm{~K}(\tilde{k})]$.

As a result, (60) is a strictly increasing function of $v$ on $\left[0, \frac{\mathrm{K}(k)}{2}\right]$.

Proof of Lemma 45. We take again $\tilde{k}=\frac{1-k^{\prime}}{1+k^{\prime}}$. By equation (26) in [14], $u_{0}(k)$ is also the unique element of $\left(-\frac{\pi}{2}, \frac{\pi}{2}\right)$ such that

$$
\sum_{j=2}^{n-1} \operatorname{sn}\left(\left(u_{0}(k)-\alpha_{j}\right)_{\tilde{k}} \mid \tilde{k}\right)=0 .
$$

Let $s_{j}=\operatorname{sn}\left(\left(u_{0}(k)-\alpha_{j}\right)_{\tilde{k}} \mid \tilde{k}\right)$. We fix an $\epsilon>0$ such that the angles $\alpha_{i}$ and $u_{0}(k)$ all lie in $\left(-\frac{\pi}{2}+\epsilon, \frac{\pi}{2}-\epsilon\right)$. Since we supposed that $u_{0}(k) \geq 0$, we have $u_{0}(k)-\alpha_{j} \epsilon$ $\left[-\frac{\pi}{2}+\epsilon, \pi-\epsilon\right]$. As a result, $\left(u_{0}(k)-\alpha_{j}\right)_{\tilde{k}} \in\left[-\mathrm{K}(\tilde{k})+\epsilon_{\tilde{k}}, 2 \mathrm{~K}(\tilde{k})-\epsilon_{\tilde{k}}\right]$. By the properties of the sn function, this implies that $s_{j}<0$ when $\alpha_{j}>u_{0}(k)$, that $s_{j}>0$ when $\alpha_{j}<u_{0}(k)$, and that $s_{j}=0$ when $\alpha_{j}=u_{0}(k)$. As a result,

$$
\sum_{j \mid \alpha_{j}<u_{0}(k)} s_{j}=\sum_{j \mid \alpha_{j}>u_{0}(k)}\left(-s_{j}\right)
$$


where all the terms in the sums are positive. In particular,

$$
\sum_{j \mid \alpha_{j}<u_{0}(k)-\frac{\pi}{2}} s_{j} \leq \sum_{j \mid \alpha_{j}>u_{0}(k)}\left(-s_{j}\right) .
$$

When $\alpha_{j}<u_{0}(k)-\frac{\pi}{2}$, then $\left(u_{0}(k)-\alpha_{j}\right)_{\tilde{k}} \in\left[\mathrm{K}(\tilde{k}), \mathrm{K}(\tilde{k})+\left(u_{0}(k)-\epsilon\right)_{\tilde{k}}\right)$. Since $\operatorname{sn}(\cdot, \tilde{k})$ is decreasing on $[\mathrm{K}(\tilde{k}), 2 \mathrm{~K}(\tilde{k})]$, in that case

$$
0<\operatorname{sn}\left(\mathrm{K}(\tilde{k})+\left(u_{0}(k)-\epsilon\right)_{\tilde{k}} \mid \tilde{k}\right)<s_{j} \leq 1 .
$$

When $\alpha_{j}>u_{0}(k)$, then $\left(u_{0}(k)-\alpha_{j}\right)_{\tilde{k}} \in\left(-\mathrm{K}(\tilde{k})+\left(u_{0}(k)+\epsilon\right)_{\tilde{k}}, 0\right)$. Since $\operatorname{sn}(\cdot, \tilde{k})$ is increasing on $[-\mathrm{K}(\tilde{k}), 0]$ and odd, in that case

$$
0<-s_{j}<\operatorname{sn}\left(\mathrm{K}(\tilde{k})-\left(u_{0}(k)+\epsilon\right)_{\tilde{k}} \mid \tilde{k}\right)
$$

Moreover, using again the symmetry and monotonicity of the sn function,

$$
\begin{aligned}
\operatorname{sn}\left(\mathrm{K}(\tilde{k})-\left(u_{0}(k)+\epsilon\right)_{\tilde{k}} \mid \tilde{k}\right) & =\operatorname{sn}\left(2 \mathrm{~K}(\tilde{k})-\left(\mathrm{K}(\tilde{k})-\left(u_{0}(k)+\epsilon\right)_{\tilde{k}}\right) \mid \tilde{k}\right) \\
& =\operatorname{sn}\left(\mathrm{K}(\tilde{k})+\left(u_{0}(k)+\epsilon\right)_{\tilde{k}} \mid \tilde{k}\right) \\
& <\operatorname{sn}\left(\mathrm{K}(\tilde{k})+\left(u_{0}(k)-\epsilon\right)_{\tilde{k}} \mid \tilde{k}\right) .
\end{aligned}
$$

As a result, we get the following inequalities:

$$
\begin{aligned}
& \operatorname{sn}\left(\mathrm{K}(\tilde{k})+\left(u_{0}(k)-\epsilon\right)_{\tilde{k}} \mid \tilde{k}\right) \#\left\{j \in[2, n-1] \mid \alpha_{j}<u_{0}(k)-\frac{\pi}{2}\right\} \\
& \quad \leq \sum_{j \mid \alpha_{j}<u_{0}(k)-\frac{\pi}{2}} s_{j} \\
& \quad \leq \sum_{j \mid \alpha_{j}>u_{0}(k)}\left(-s_{j}\right) \\
& \quad \leq \operatorname{sn}\left(\mathrm{K}(\tilde{k})-\left(u_{0}(k)+\epsilon\right)_{\tilde{k}} \mid \tilde{k}\right) \#\left\{j \in[2, n-1] \mid \alpha_{j}>u_{0}(k)\right) \\
& \quad<\operatorname{sn}\left(\mathrm{K}(\tilde{k})+\left(u_{0}(k)-\epsilon\right)_{\tilde{k}} \mid \tilde{k}\right) \#\left\{j \in[2, n-1] \mid \alpha_{j}>u_{0}(k)\right) .
\end{aligned}
$$

In the last inequality, we used the fact that the cardinal is not zero since these $j$ are exactly those that give a negative term in in (61); those negative terms have to exist because the $\alpha_{j}$ are not all equal. Dividing by $\operatorname{sn}\left(\mathrm{K}(\tilde{k})+\left(u_{0}(k)-\epsilon\right)_{\tilde{k}} \mid \tilde{k}\right)>0$, we get the claim of Lemma 45. 


\section{References}

1. Abramowitz, M., Stegun, I.A.: Handbook of Mathematical Functions with Formulas, Graphs, and Mathematical Tables. Dover, New York (1964). ninth Dover printing, tenth GPO printing edition

2. Basok, M., Chelkak, D.: Tau-functions à la Dubédat and probabilities of cylindrical events for doubledimers and CLE(4). arXiv preprint arXiv:1809.00690 (2018)

3. Baxter, R., Perk, J., Au-Yang, H.: New solutions of the star-triangle relations for the chiral Potts model. Phys. Lett. A 128(3), 138-142 (1988)

4. Baxter, R.J.: Partition function of the eight-vertex lattice model. Ann. Phys. 70(1), 193-228 (1972)

5. Baxter, R.J.: Solvable eight-vertex model on an arbitrary planar lattice. Philos. Trans. R. Soc. Lond. A Math. Phys. Eng. Sci. 289(1359), 315-346 (1978)

6. Baxter, R.J.: Exactly Solved Models in Statistical Mechanics. Academic Press, London (1982)

7. Baxter, R.J.: Free-fermion, checkerboard and Z-invariant lattice models in statistical mechanics. Proc. R. Soc. Lond. A 404(1826), 1-33 (1986)

8. Bazhanov, V.V., Stroganov, Y.G.: Hidden symmetry of free Fermion model. Theor. Math. Phys. 62(3), 253-260 (1985)

9. Bazhanov, V.V., Stroganov, Y.G.: Hidden symmetry of free Fermion model. II. Partition function. Theor. Math. Phys. 63(2), 519-527 (1985)

10. Bazhanov, V.V., Stroganov, Y.G.: Hidden symmetry of free Fermion model. III. Inversion relations. Theor. Math. Phys. 63(3), 604-611 (1985)

11. Belavin, A.: Exact solution of the two-dimensional model with asymptotic freedom. Phys. Lett. B 87(1), 117-121 (1979)

12. Boutillier, C., de Tilière, B.: The critical Z-invariant Ising model via dimers: the periodic case. Probab. Theory Relat. Fields 147(3), 379-413 (2010)

13. Boutillier, C., de Tilière, B.: The critical Z-invariant Ising model via dimers: locality property. Commun. Math. Phys. 301(2), 473-516 (2011)

14. Boutillier, C., de Tilière, B., Raschel, K.: The Z-invariant massive Laplacian on isoradial graphs. Invent. Math. 208(1), 109-189 (2017)

15. Boutillier, C., de Tilière, B.: Height representation of XOR-Ising loops via bipartite dimers. Electron. J. Probab. 19, 33 (2014)

16. Boutillier, C., de Tilière, B., Rasche, K.: The Z-invariant Ising model via dimers. Probab. Theory Relat. Fields 174, 235-305 (2019)

17. Carlson, B., Todd, J.: The degenerating behavior of elliptic functions. SIAM J. Numer. Anal. 20(6), $1120-1129(1983)$

18. Chelkak, D., Cimasoni, D., Kassel, A.: Revisiting the combinatorics of the 2D Ising model. Annales de l'Institut Henri Poincaré D 4(3), 309-385 (2017)

19. Cimasoni, D., Duminil-Copin, H.: The critical temperature for the Ising model on planar doubly periodic graphs. Electron. J. Probab. 18, 44 (2013)

20. Cimasoni, D., Reshetikhin, N.: Dimers on surface graphs and spin structures. I. Commun. Math. Phys. 275(1), 187-208 (2007)

21. Cohn, H., Kenyon, R., Propp, J.: A variational principle for domino tilings. J. Am. Math. Soc. 14(2), 297-346 (2001)

22. de Tilière, B.: Quadri-tilings of the plane. Probab. Theory Relat. Fields 137(3), 487-518 (2007)

23. de Tilière, B.: The $Z$-Dirac and massive Laplacian operators in the $Z$-invariant Ising model. arXiv e-prints (2018)

24. Dolbilin, N.P., Mishchenko, A.S., Shtan'ko, M.A., Shtogrin, M.I., Zinoviev, Y.M.: Homological properties of dimer configurations for lattices on surfaces. Funct. Anal. Appl. 30(3), 163-173 (1996)

25. Dubédat, J.: Double dimers, conformal loop ensembles and isomonodromic deformations. J. Eur. Math. Soc. (JEMS) 21(1), 1-54 (2019)

26. Dubédat, J.: Exact bosonization of the Ising model. arXiv:1112.4399v1 (2011)

27. Dubédat, J.: Topics on abelian spin models and related problems. Probab. Surv. 8, 374-402 (2011)

28. Duminil-Copin, H., Li, J.-H., Manolescu, I.: Universality for the random-cluster model on isoradial graphs. Electron. J. Probab. 23, 70 (2018)

29. Dutyshev, V.N.: Two-dimensional isotopic model of a fermion field with broken SU(2) symmetry. Sov. J. Exp. Theor. Phys. 51, 671 (1980)

30. Fan, C., Wu, F.Y.: General lattice model of phase transitions. Phys. Rev. B 2, 723-733 (1970)

31. Felderhof, B.: Diagonalization of the transfer matrix of the free-Fermion model. II. Physica 66(2), 279297 (1973)

32. Felderhof, B.: Diagonalization of the transfer matrix of the free-Fermion model. III. Physica 66(3), 509526 (1973)

33. Felderhof, B.: Direct diagonalization of the transfer matrix of the zero-field free-Fermion model. Physica 65(3), 421-451 (1973) 
34. Fisher, M.E.: On the dimer solution of planar Ising models. J. Math. Phys. 7(10), 1776-1781 (1966)

35. Galleas, W., Martins, M.J.: Yang-Baxter equation for the asymmetric eight-vertex model. Phys. Rev. E 66(4), 047103 (2002)

36. Galluccio, A., Loebl, M.: On the theory of Pfaffian orientations. I. Perfect matchings and permanents. Electron. J. Comb. 6, R6 (1999)

37. Hsue, C.S., Lin, K.Y., Wu, F.Y.: Staggered eight-vertex model. Phys. Rev. B 12, 429-437 (1975)

38. Jordan, H.: Eine Bemerkung Über die Monotonie von sn(tK). Arch. Math. 6(3), 185-187 (1955)

39. Kac, M., Ward, J.C.: A combinatorial solution of the two-dimensional Ising model. Phys. Rev. 88, 13321337 (1952)

40. Kadanoff, L.P., Ceva, H.: Determination of an operator algebra for the two-dimensional Ising model. Phys. Rev. B 3, 3918-3939 (1971)

41. Kasteleyn, P.W.: The statistics of dimers on a lattice: I. The number of dimer arrangements on a quadratic lattice. Physica 27, 1209-1225 (1961)

42. Kasteleyn, P.W.: Dimer statistics and phase transitions. J. Math. Phys. 4(2), 287-293 (1963)

43. Kasteleyn, P. W.: Graph theory and crystal physics. In: Graph Theory and Theoretical Physics, pp 43-110 (1967)

44. Kenyon, R.: Local statistics of lattice dimers. Annales de l'Institut Henri Poincare (B) Probability and Statistics 33(5), 591-618 (1997)

45. Kenyon, R.: The Laplacian and Dirac operators on critical planar graphs. Invent. Math. 150(2), 409-439 (2002)

46. Kenyon, R.: Dimer Problems. In: Françoise, J.-P., Naber, G.L., Tsun, T.S. (eds.) Encyclopedia of Mathematical Physics. Academic Press, Amsterdam (2006)

47. Kenyon, R.: Conformal invariance of loops in the double-dimer model. Commun. Math. Phys. 326, 477-497 (2011)

48. Kenyon, R., Okounkov, A., Sheffield, S.: Dimers and amoebae. Ann. Math. 163, 1019-1056 (2006)

49. Kenyon, R., Schlenker, J.-M.: Rhombic embeddings of planar quad-graphs. Trans. Am. Math. Soc. 357(9), 3443-3458 (2005)

50. Khachatryan, S., Sedrakyan, A.: On the solutions of the Yang-Baxter equations with general inhomogeneous eight-vertex R-Matrix: relations with Zamolodchikov's tetrahedral algebra. J. Stat. Phys. 150(1), $130-155$ (2013)

51. Kramers, H.A., Wannier, G.H.: Statistics of the two-dimensional ferromagnet. Part I. Phys. Rev. 60, 252-262 (1941)

52. Lawden, D.F.: Jacobi's Elliptic Functions. Springer, New York (1989)

53. Lieb, E.H.: Residual entropy of square ice. Phys. Rev. 162, 162-172 (1967)

54. Lin, K.Y.: Staggered eight-vertex model on the Kagome lattice. J. Phys. A Math. Gen. 9(4), 581 (1976)

55. Lin, K.Y.: Eight-vertex model on a ruby lattice. J. Phys. A Math. Gen. 17(16), 3201 (1984)

56. Lin, K.Y., Wang, I.P.: Staggered eight-vertex model with four sublattices. J. Phys. A Math. Gen. 10(5), 813 (1977)

57. Mercat, C.: Discrete Riemann surfaces and the Ising model. Commun. Math. Phys. 218(1), 177-216 (2001)

58. Nienhuis, B.: Critical behavior of two-dimensional spin models and charge asymmetry in the Coulomb gas. J. Stat. Phys. 34(5), 731-761 (1984)

59. Perk, J.H.H., AuYang, H.: Yang-Baxter equations. In: Françoise, J.P., Naber, G.L., Tsou, S.T. (eds.) Encyclopedia of Mathematical Physics, vol. 5, pp. 465-473. Elsevier, Amsterdam (2006)

60. Saunders, B.D., Schneider, H.: Flows on graphs applied to diagonal similarity and diagonal equivalence for matrices. Discrete Math. 24(2), 205-220 (1978)

61. Slater, J.C.: Theory of the transition in KH2PO4. J. Chem. Phys. 9(1), 16-33 (1941)

62. Sogo, K., Uchinami, M., Akutsu, Y., Wadati, M.: Classification of exactly solvable two-component models. Progress Theoret. Phys. 68, 508-526 (1982)

63. Sutherland, B.: Two-dimensional hydrogen bonded crystals without the ice rule. J. Math. Phys. 11(11), 3183-3186 (1970)

64. Temperley, H.N.V., Fisher, M.E.: Dimer problem in statistical mechanics-an exact result. Philos. Mag. A J. Theor. Exp. Appl. Phys. 6(68), 1061-1063 (1961)

65. Tesler, G.: Matchings in graphs on non-orientable surfaces. J. Comb. Theory Ser. B 78(2), 198-231 (2000)

66. Vieira, R.S.: Solving and classifying the solutions of the Yang-Baxter equation through a differential approach. Two-state systems. J. High Energy Phys. 2018, 110 (2018)

67. Wegner, F.J.: Duality relation between the Ashkin-Teller and the eight-vertex model. J. Phys. C Solid State Phys. 5(11), L131 (1972)

68. Wu, F.Y.: Exact solution of a model of an antiferroelectric transition. Phys. Rev. 183, 604-607 (1969)

69. Wu, F.Y., Lin, K.Y.: Staggered ice-rule vertex model-the Pfaffian solution. Phys. Rev. B 12, 419-428 (1975) 
70. Zamolodchikov, A.B.: Z4-symmetric factorizeds-matrix in two space-time dimensions. Commun. Math. Phys. 69(2), 165-178 (1979)

Communicated by P. Di Francesco 